\title{
Functional renormalization group approach to electronic structure calculations for systems without translational symmetry
}

\author{
Christian Seiler and Ferdinand Evers* \\ Institut für Theoretische Physik, Universität Regensburg, D-93053 Regensburg, Germany \\ (Received 14 May 2016; revised manuscript received 19 August 2016; published 3 October 2016)
}

\begin{abstract}
A formalism for electronic-structure calculations is presented that is based on the functional renormalization group (FRG). The traditional FRG has been formulated for systems that exhibit a translational symmetry with an associated Fermi surface, which can provide the organization principle for the renormalization group (RG) procedure. We here advance an alternative formulation, where the RG flow is organized in the energy-domain rather than in $k$ space. This has the advantage that it can also be applied to inhomogeneous matter lacking a band structure, such as disordered metals or molecules. The energy-domain FRG $(\epsilon$ FRG) presented here accounts for Fermi-liquid corrections to quasiparticle energies and particle-hole excitations. It goes beyond the state of the art $G W$-BSE, because in $\epsilon$ FRG the Bethe-Salpeter equation (BSE) is solved in a self-consistent manner. An efficient implementation of the approach that has been tested against exact diagonalization calculations and calculations based on the density matrix renormalization group is presented. Similar to the conventional FRG, also the $\epsilon$ FRG is able to signalize the vicinity of an instability of the Fermi-liquid fixed point via runaway flow of the corresponding interaction vertex. Embarking upon this fact, in an application of $\epsilon$ FRG to the spinless disordered Hubbard model we calculate its phase boundary in the plane spanned by the interaction and disorder strength. Finally, an extension of the approach to finite temperatures and spin $S=1 / 2$ is also given.
\end{abstract}

DOI: 10.1103/PhysRevB.94.155102

\section{INTRODUCTION}

Correlation effects are the driving agent behind a great many of the phenomena that are comprising the contemporary physics of condensed matter systems. As long as interactions are not too strong, such correlation phenomena can be understood in terms of an effective single particle picture as it is provided, e.g., by the Fermi-liquid theory. In this weakly correlated limit, the density-functional theory (DFT) can yield useful, often quantitative results for the electronic structure of crystalline or molecular matter. Where DFT fails to be quantitative, post-DFT correction schemes have been introduced that can significantly improve the accuracy, in particular with respect to (charged) excitation energies [1]. As a particularly successful example, we mention the $G W$ approximation motivated by conventional diagrammatic perturbation theory [2-4].

At low enough temperatures, most real materials undergo a transition into a correlated low-temperature phase, such as a magnet or a superconductor. Such phenomena are usually at the verge of applicability of perturbative methods. Still, perturbation theory can be very useful, because it often signalizes the existence of such phase transitions via divergent diagrams. In recent years a powerful method has been devised to deal with stronger correlations, the functional renormalization group (FRG), that has proven particularly successful in this respect [5,6]. It can be (roughly) thought of as a systematic extension of $G W$ theory and its Bethe-Salpetertype generalizations. ${ }^{1}$ Because it monitors the RG flow of a representative set of interaction vertices, FRG can predict in an unbiased way the leading Fermi liquid instabilities together with estimates for the corresponding phase boundaries.

\footnotetext{
*Corresponding author: ferdinand.evers@ur.de

${ }^{1}$ See, e.g., Refs. [57,58].
}

Beyond phase boundaries, the FRG is capable to predict a variety of other physical observables including Luttingerliquid parameters [7], Fermi-liquid corrections [8,9], and spin susceptibilities [10-12]. Correspondingly, the FRG has been applied to a variety of systems, e.g., the Hubbard model in various parameter regimes [13-19], single impurity models [20,21], spin [10-12,22-24] and quantum critical systems [25-27]. A generalized version of the method has been devised $[28,29]$ so that also broken symmetry phases could be addressed such as superfluids and superconductors [29-31]. For an overview, we direct the reader to Refs. [5,32].

Several formulations of FRG have been devised to treat various situations with broken translational invariance: single impurity models have been investigated in the context of Luttinger liquids [33,34] and the Kondo-effect [35]; for a review, see Ref. [5]. The transport through a quantum pointcontact with spatial structure has been analyzed by Bauer et al. [36,37]. The magnetization of graphene based nanodisks has been studied in the presence of zero-energy states that are supported by the system geometry [38]. Effective field theories of disordered systems have also been addressed. Katanin has worked out an FRG scheme for noninteracting electrons and applied it to graphene [39]. Even earlier, Dusuel and Zanchi used an FRG-type approach for analyzing the interplay of disorder and superconductivity [40].

Explicit FRG treatments of individual inhomogeneous systems as we have them in mind here, such as molecules or disordered metals are still scarce. The work by Karrasch and Moore probably comes closest who consider disordered Luttinger liquids [41].

\section{A. Motivation underlying this work}

Good progress has been made in electronic structure calculations for real materials as well as for model Hamiltonians. Still, we believe that there is room for improvement. With an 
eye on $a b$ initio calculations, we observe that it is still very challenging to accurately calculate, e.g., ionization energies and electron affinities of small molecules or atom clusters. Quantitative results from DFT can be obtained only via procedures, such as $\triangle \mathrm{SCF}$, that rely on error cancellation. The $G_{0} W_{0}$ method in this respect seems more reliable; benchmarks for different implementations have recently become available $[4,42,43]$. The $G_{0} W_{0}$ approximation is not self-consistent, however, and partly for this reason it comes in many flavors. The development and testing of self-consistent and computationally affordable $G W$ schemes is currently under way [43-46]. Even more challenging it is to calculate the dynamical response, e.g., the optical gap or the absorption spectrum. The traditional time-dependent DFT, such as TDLDA, tends to underestimate optical gaps in solids by $\sim e V$. Interestingly, it can quantitatively reproduce excitation gaps of small molecules when combined with long-range functionals, especially if they are optimally tuned $[47,48]$.

In combination with $G W$ theory, one solves the BetheSalpeter equation to find the optical properties. Due to the computational complexity, one usually keeps only the simplest nontrivial vertex corrections $(G W+\mathrm{BSE})$. The approach yields results often with a typical accuracy of a few hundred meV, see Ref. [49] for a recent overview and Ref. [50] for benchmarks. In some cases much larger deviations have been reported, however, calling for a further validation of $G W+\mathrm{BSE}$ [51]. State of the art $G W$ implementations can be found in many standard band structure codes, e.g., Refs. [52-56].

(i) In this situation, it seems advisable to go a step forward and explore more complete approximation schemes that in principle could go significantly beyond the lowest order BSE-technology by incorporating, e.g., a self-consistent evaluation of screening in the presence of vertex corrections. The extended scheme would thus provide a laboratory for testing the current BSE-technology against a more accurate higher order method. In accord with earlier work [57,58], also our effort is underlying the idea that the FRG could be an interesting candidate for a more advanced electronic structure theory.

A certain limitation of the FRG in its most common flavor is that it was formulated having homogeneous systems in mind. It thus could form the basis for improved band structure calculations for crystalline matter, but it will be inefficient with more inhomogeneous systems that we are mostly interested in, here. Having in mind the program outlined before in (i), we consider it an interesting challenge modifying the traditional $k$-space FRG ( $k$ FRG) into a new tool-energy-domain FRG $(\epsilon \mathrm{FRG})$ - that can also describe the phases and the corresponding transitions in weakly correlated, inhomogeneous matter.

(ii) To elaborate on the perspective for the $\epsilon \mathrm{FRG}$, we mention two research fields with prospective applications. (1) Quantum chemistry calculations could benefit from $\epsilon$ FRG in a range of system sizes where high-precision calculations, e.g., the couple-cluster approach, are computationally not affordable any more. (2) The $\epsilon$ FRG might prove a useful tool for investigating the effect that disorder has on those quantum phase transitions that have already been investigated in the clean limit [5]. Conversely, there is the intriguing prospect to study the effect that weak interactions have on disordered systems with wave functions that are localized due to quantum interference [59].

Motivated by (2), we here present an implementation of an $\epsilon \mathrm{FRG}$ that can operate on disordered model Hamiltonians. Our goal is to explore the potential of the approach as a higher-order method for studies of weakly correlated fermions in generic environments lacking translational symmetries.

\section{B. FRG for systems without translational symmetries: $\epsilon$ FRG}

Consider a fermion system with a Hamiltonian that decomposes into a one-body and a two-body parts,

$$
\hat{H}=\hat{H}_{0}+\hat{U} \text {. }
$$

The noninteracting part $\hat{H}_{0}$ includes a static potential. It is considered generic in the sense that it does not exhibit translational symmetries; its single-particle eigenstates $|\alpha\rangle, \alpha=1, \ldots, N$ are far from plane waves. They can be thought of as wave functions of a strongly disordered metal or as molecular orbitals, e.g., of a generic organic molecule. We will leave the interacting part $\hat{U}$ unspecified for the time being.

\section{Excursion: Hedin's equations and FRG}

As was recognized by L. Hedin, in order to compute physical observables in the presence of two-body interactions, one can solve a set of self-consistent nonlinear matrix equations for the exact (causal) Green's function, the corresponding self-energies and vertex functions [60,61]. Unfortunately, Hedin's equations are impossible to solve exactly even with today's computational resources for realistic system sizes. Difficulties arise because of (a) the complicated nature of the matrix kernels and (b) the very large dimensions of the matrices involved, especially of the interaction vertex $\Gamma$. The ubiquitous approximation strategy therefore is truncating the matrix equations so that the kernels simplify and reducing the effective matrix size by grading the many-particle Hilbert space. Eventually, also the FRG relies on such a truncation scheme.

However, even the truncated set of equations is very difficult to solve. Partially, this is because the requirement of the solution being self-consistent. Here the idea of the renormalization group (RG) with the corresponding flow-equation comes in. Speaking in a lose manner, what corresponds to an iteration cycle in conventional solutions of self-consistency problems is in the framework of FRG replaced by a consecutive integration of a differential equation that establishes the $\mathrm{RG}$ flow. The initializing guess of the iteration cycle corresponds to the initialization of the flow equation; the flow stops once the (self-consistent) fixed-point has been reached. ${ }^{2}$ Advantages of the RG approach over self-consistency cycles are (a) that uncertainties related to the proper choice of the starting guess

\footnotetext{
${ }^{2}$ Strictly speaking, the RG flow arrives at a self-consistent fixedpoint only for certain (conserving) truncation schemes of the RG equations. A well known scheme satisfying this criterion has been devised by Katanin [94]. Unfortunately, implementing the Katanin scheme is computationally so expensive that we here have to refrain from doing so.
} 

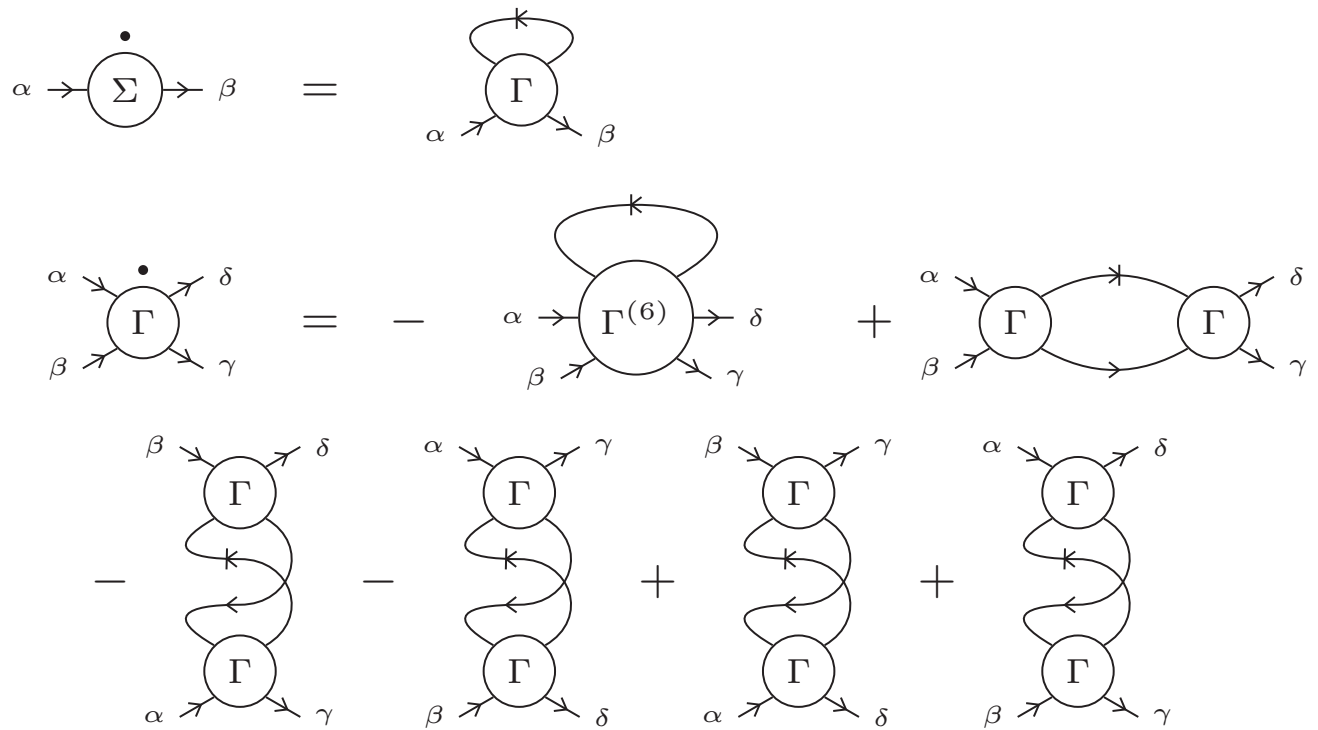

FIG. 1. Diagrammatic representation of the FRG flow equations for the self-energy $\Sigma^{\Lambda}$ and the vertex $\Gamma^{\Lambda}$. A vertical bar denotes the single-scale propagator $\mathcal{S}^{\Lambda}$, the other propagators are $\mathcal{G}^{\Lambda}$. As usual, external legs do not entail a propagator.

are removed and (b) there is a clear physical interpretation in terms of "runaway flow" even when the numerical integration breaks down, so the RG-flow cannot be followed all the way to the fixed-point. In contrast, the lack of convergence of a self-consistency cycle is much more difficult to interpret consistently.

\section{Mathematical challenges of FRG}

For the specific set of flow equations used in this work, we adopt the same truncation scheme for the RG equations, Fig. 1, that also is underlying the traditional FRG for periodic systems $(k \mathrm{FRG})$. At this stage the only difference is that with $\epsilon$ FRG each line represents a (Matsubara) Green's function deriving from a resolvent $G=\left(\mathrm{i} \omega-H_{0}\right)^{-1}$ that is not diagonal in momentum $(k-)$ space. Figure 1 gives a graphical representation of a set of nonlinear (integro-)differential equations that represent a typical initial-value problem; the flowing energy cutoff $\Lambda$ plays a role analogous to a time. Ideally, after integrating the equations from $\Lambda=\infty$ to $\Lambda=0$ an exact solution of the (truncated) vertex equation has been found.

As we already mentioned, solving the truncated set of flow equations, Fig. 1, still poses a problem of formidable computational complexity. The difficulty arises from the fact that the vertex function, $\Gamma(\Omega)$, is represented as very large family of matrices with three continuous frequencies, $\Omega=\left(\omega_{1}, \omega_{2}, \omega_{3}\right)$, acting as family parameters. In addition, each matrix has four indices, every one of which explores, in principle, the basis set of the full single-particle Hilbert space.

\section{Established approximation strategies}

Several simplification strategies can reduce the computational effort, making FRG feasible and competitive. We offer a short overview.

a. Static (or adiabatic) approximation. The frequency dependence of the vertex function is neglected, $\Gamma\left(\omega_{1}, \omega_{2} ; \omega_{1}^{\prime}\right) \rightarrow$
$\Gamma(0)$. This is analogous to the static screening approximation familiar from the traditional treatment of the BSE imposed on top of $G_{0} W_{0}$ [62-64]. In FRG, one also ignores the frequency dependency of the self-energy $\Sigma$. As a consequence, $\Sigma_{\text {FRG }}$ turns into an energy-independent, Hermitian correction to the reference Hamiltonian $\hat{H}_{0}$. The effective Hamiltonian matrix $H_{\text {eff }}=H_{0}+\Sigma_{\mathrm{FRG}}$ defines the quasiparticle energies and wave functions. With respect to the static self-energy, the situation in FRG is completely analogous to the one in the quasiparticle self-consistent $G W$ theory (qp $G W$ ) [46,65-68]. The advantage of FRG over this theory is, that vertex corrections are accounted for in FRG in a self-consistent manner.

In the static approximation, the scaling of FRG with the dimension of the single-particle Hilbert space, $N$, is formally $N^{6}$ if one does not consider further symmetries such as translational invariance. It is thus roughly comparable to the scaling of high-precision methods in quantum chemistry, like the coupled cluster method (flavor CCSD) [69].

b. Clean systems: Fermi-surface projection and channel decomposition in $k F R G$. In the clean case, $H_{0}$ exhibits a translational symmetry, so the number of independent matrix elements of $\Gamma(0)$ reduces significantly. Moreover, a Fermisurface exists that helps to identify a hierarchical structure within the matrix elements of $\Gamma(0)$. In many cases, only matrix elements with wave vectors close to the Fermi surface dominate the physics of the system, so the vertex at momenta away from this surface may be replaced by the vertex with momenta projected onto it, drastically simplifying the calculation. (" $N$-patching" scheme)

Similar to the standard procedure in fermionic field theories [70], one can efficiently parametrize the vertex function splitting it into three different interaction channels by analyzing the phase-space of $k$-space scattering [71]. Each channel traces three possible transitions triggered by charge, spin or superconducting fluctuations. 
The channel decomposition is an attractive alternative to the patching procedure: it is physically transparent and therefore simplifies the analysis of runaway flow. Due to its computational efficiency, an explicit dependency in each channel on one frequency can be kept. Finally, in its extended versions the flow can be followed all the way into the symmetry-broken phases [28,29].

The conventional rationale motivating the channel decomposition relies on phase-space arguments for scattering events in $k$ space near the Fermi-surface. This seems to suggest that channel decomposition is well motivated for clean and weakly inhomogeneous systems.

c. Localizing the vertex function in real space. Several authors $[36,37,41]$ have adopted an approximation that neglects (all or a subset of) nonlocal terms in the flow equation for the vertex function. Thus the vertex equations when formulated in real-space simplify significantly. The method should be applicable if long-range correlations in the interaction vertex remain unimportant for the critical physics near the phase boundary.

\section{4. "Active-space" approximation for $\epsilon F R G$}

In the case of generic systems, there is no intrinsic symmetry guidance as towards how to simplify the matrix structure of $\Gamma(0)$. In particular, Fermi-surface projection is not feasible. The most important new conceptual step in $\epsilon$ FRG as compared to $k \mathrm{FRG}$ will be to find an alternative to the common Fermi-surface projection. It should reduce the number of degrees of freedom that are kept explicit in the RG calculation without invoking a momentum-space concept. In this work, we propose and test an "active-space" approximation (ASA) that can achieve this goal.

The ASA could be a potential alternative working well in strongly inhomogeneous situations where due to the complete absence of a Fermi-sphere concept a channel decomposition might no longer be meaningful. We will develop it here in a version that keeps the full spatial dependency of the interaction vertex avoiding to localize the vertex equations. To be able to do this, the static approximation had to be adopted. This is a certain drawback against the method devised by Bauer et al. that employs a channel decomposition with (residual) frequency dependency in the (localized) vertex equation [37].

a. How to choose the active space. Similar to the FRGtreatment of clean systems, we also work in the eigenstate basis $|\alpha\rangle$ of the noninteracting Hamiltonian $\hat{H}_{0}$. Then the vertex function takes a matrix representation $\Gamma_{\alpha_{1}, \alpha_{2}, \alpha_{3}, \alpha_{4}}(0)$. To simplify the flow equations, we will approximate this matrix by the bare interaction vertex, $U_{\alpha_{1}, \alpha_{2}, \alpha_{3}, \alpha_{4}}$, whenever one of the states $\left|\alpha_{i}\right\rangle, i=1, \ldots, 4$ is outside a certain active space $\mathcal{H}_{M}$ of the full single-particle Hilbert space, $\mathcal{H}$. A natural choice of $\mathcal{H}_{M}$ corresponds to states with energy $\epsilon_{\alpha}$ in the vicinity of the chemical potential $\mu_{\text {chem. }}$. The index $M$ indicates the size of the volume, which could be characterized by an energy scale or simply by the number of states that it contains. We will adopt the simplest choice associating $M$ with the number of states kept in $\mathcal{H}_{M}$.

b. Computational scaling. The important computational aspect of the active-space concept is that it brings the nominal scaling of $\epsilon$ FRG down to $M^{4} N^{2}+M^{2} N^{3}$. The optimal choice of $M$ balances the computational effort against the required numerical accuracy of the calculational results. In our applications, we found that typically $M=N / 3$ is a reliable choice. It is implying a speedup of a factor $10^{2}$ for the applications that we have investigated. For the limit of large $N$, we argue that $M \sim N^{1 / 2}$ in two-dimensional systems, so that the net scaling of $\epsilon$ FRG would be $N^{4}$. It thus formally scales comparable to current implementations of the $G W$ method.

\section{Application of $\epsilon$ FRG: disordered Hubbard model}

As a first application of the new formalism and in order to demonstrate what can be achieved with it, we have studied the 2D spinless, repulsive Hubbard model with on-site disorder at half filling. At zero disorder, $W=0$, the model exhibits a charge-density wave, while at zero interaction, $U=0$, the ground state corresponds to an Anderson insulator. Our interest is in determining the phase boundary that separates the two phases in the situation where disorder and interaction compete. We have calculated it in the $U / W$ plane. Specifically, we can establish that at $W>0$, the Anderson insulator survives as long as the interaction does not exceed a critical value, $U>U^{*}(W)>0$.

\section{Conclusion and outlook}

As it is typical with higher-order methods, the computational bottleneck restricts the feasible system sizes. In our applications, we found it practical to work with a single particle Hilbert space containing $N \sim 50-100$ states. Our preliminary tests indicate that substantially bigger system sizes of a few hundred states are realistically accessible, $N \sim 200-400$, after additional improvements in the code performance have been implemented. It is only the limit of very large values of $N$, though, where the superior scaling of $\epsilon \mathrm{FRG}$ will become effective, so that the method becomes favorable as compared to other well established techniques, such as CCSD or quantum Monte Carlo. Whether these system sizes actually can be reached, future research will tell. At present, $\epsilon$ FRG is readily applicable to models of interacting fermions in low dimensions, which includes Hubbard models with spin and (attractive) interactions at different filling fractions, but also, e.g., small molecules.

\section{E. Organization of this paper}

The paper is organized in the following way. In Sec. II, we give the main formalism including the formulæ needed to reconstruct physical observables, in particular densities and occupation numbers. Also the formulæ for the finitetemperature formalism are given there, so that also, e.g., the effect of heat could be studied. Section III provides the computational details of our specific implementation of the main formulæ. In the consecutive section, Sec. IV, we test this implementation on 1D- and 2D-model systems of disordered fermions against numerically exact results from exact diagonalization and the density matrix renormalization group (DMRG) for small system sizes.

To illustrate the potential of $\epsilon$ FRG, we present in Sec. V an application to the disordered, spinless 2D-Hubbard model. We will calculate and discuss the phase boundary between the 
Anderson insulator and the Mott phase in the plane spanned by the disorder and interaction strength.

\section{GENERAL METHODOLOGY OF $\epsilon$ FRG}

In this section, we will develop our $\epsilon$ FRG scheme. We will assume that it is practical to diagonalize the noninteracting Hamiltonian exactly,

$$
\hat{H}_{0}|\alpha\rangle=\epsilon_{\alpha}|\alpha\rangle
$$

yielding eigenstates $\{|\alpha\rangle\}$ with corresponding eigenenergies $\left\{\epsilon_{\alpha}\right\}$. This allows us to rewrite the full Hamiltonian in terms of the noninteracting eigenbasis,

$$
\hat{H}=\sum_{\alpha} \epsilon_{\alpha} \hat{\mathbf{c}}_{\alpha}^{\dagger} \hat{\mathbf{c}}_{\alpha}+\frac{1}{4} \sum_{\alpha \beta \gamma \delta} U_{\alpha \beta \gamma \delta} \hat{\mathbf{c}}_{\alpha}^{\dagger} \hat{\mathbf{c}}_{\beta}^{\dagger} \hat{\mathbf{c}}_{\delta} \hat{\mathbf{c}}_{\gamma} .
$$

Here, $U_{\alpha \beta \gamma \delta}$ are the antisymmetrized bare interaction matrix elements in the noninteracting eigenbasis.

As discussed in Ref. [5], the FRG is a means to solve this interacting problem by introducing a cutoff into the bare propagator of the system. As the systems we want to study are inhomogeneous in nature, and hence the single-particle states are not easily classified systematically, we introduce a cutoff in frequency space (as opposed to momentum space), see Eq. (57) in Ref. [5],

$$
\mathcal{G}^{0, \Lambda}(\mathrm{i} \omega)=\frac{\Theta^{\Lambda}(\omega)}{\mathrm{i} \omega-H_{0}+\mu_{\text {chem }}},
$$

where $\Theta^{\Lambda}(\omega)$ vanishes at $\Lambda \rightarrow \infty$ and approaches 1 at $\Lambda \rightarrow 0$; see below for a discussion of our choice for $\Theta^{\Lambda}(\omega)$.

As a consequence of introducing the infrared cutoff, $\Lambda$, all other quantities of the system depend on $\Lambda$. If we take the limit of $\Lambda \rightarrow \infty$, it can be shown (see Eq. (31) in Ref. [5]) that the self-energy vanishes and the effective interaction vertex $\Gamma$ is given by the matrix elements of the bare interaction, $U \ldots$. On the other hand, taking the limit of $\Lambda \rightarrow 0$, we recover the original system without the introduced cutoff. There is now a continuous variable that connects the real system $(\Lambda \rightarrow 0)$, where the physical quantities are not known a priori, with a trivial system $(\Lambda \rightarrow \infty)$, where all quantities are known.

\section{A. Flow equations}

As is discussed in the literature [5,6], the derivatives of the vertex functions (self-energy, effective interaction, etc.) yield a set of flow equations; a full derivation of their most generic form may be found in Chap. 4 of Ref. [6]. Following Ref. [5] [Eq. (50)], we will adopt the generic formulation of the flow equations,

$$
\frac{d}{d \Lambda} \Sigma^{\Lambda}\left(x^{\prime}, x\right)=\sum_{y, y^{\prime}} \mathcal{S}^{\Lambda}\left(y, y^{\prime}\right) \Gamma^{\Lambda}\left(x^{\prime}, y^{\prime} ; x, y\right),
$$

and for the vertex, Ref. [5] [Eq. (52)],

$$
\begin{aligned}
\frac{d}{d \Lambda} \Gamma^{\Lambda}\left(x_{1}^{\prime}, x_{2}^{\prime} ; x_{1}, x_{2}\right)= & \sum_{y_{1}, y_{1}^{\prime}} \sum_{y_{2}, y_{2}^{\prime}} \mathcal{G}^{\Lambda}\left(y_{1}, y_{1}^{\prime}\right) \mathcal{S}^{\Lambda}\left(y_{2}, y_{2}^{\prime}\right)\left\{\Gamma^{\Lambda}\left(x_{1}^{\prime}, x_{2}^{\prime} ; y_{1}, y_{2}\right) \Gamma^{\Lambda}\left(y_{1}^{\prime}, y_{2}^{\prime} ; x_{1}, x_{2}\right)-\left[\Gamma^{\Lambda}\left(x_{1}^{\prime}, y_{2}^{\prime} ; x_{1}, y_{1}\right) \Gamma^{\Lambda}\left(y_{1}^{\prime}, x_{2}^{\prime} ; y_{2}, x_{2}\right)\right.\right. \\
& \left.\left.+\left(y_{1} \leftrightarrow y_{2}, y_{1}^{\prime} \leftrightarrow y_{2}^{\prime}\right)\right]+\left[\Gamma^{\Lambda}\left(x_{2}^{\prime}, y_{2}^{\prime} ; x_{1}, y_{1}\right) \Gamma^{\Lambda}\left(y_{1}^{\prime}, x_{1}^{\prime} ; y_{2}, x_{2}\right)+\left(y_{1} \leftrightarrow y_{2}, y_{1}^{\prime} \leftrightarrow y_{2}^{\prime}\right)\right]\right\} \\
& -\sum_{y, y^{\prime}} \mathcal{S}^{\Lambda}\left(y, y^{\prime}\right) \Gamma^{(6), \Lambda}\left(x_{1}^{\prime}, x_{2}^{\prime}, y^{\prime} ; x_{1}, x_{2}, y\right)
\end{aligned}
$$

Here, $x$ and $y$ are combined indices for space and time coordinates. A diagrammatic representation of these equations is given in in Fig. 1. Furthermore, we copy the definition of Ref. [5] [Eq. (47)] for the single-scale propagator,

$$
\mathcal{S}^{\Lambda}=-\mathcal{G}^{\Lambda}\left[\frac{d}{d \Lambda}\left(\mathcal{G}^{0, \Lambda}\right)^{-1}\right] \mathcal{G}^{\Lambda}
$$

We next rewrite these quantities into our own nomenclature, where we work in Matsubara space. Furthermore, we separate the generic indices into Matsubara frequencies and Hilbert space indices, $x \rightarrow\left(\mu, \omega_{n}\right)$. We also drop the term with $\Gamma^{(6), \Lambda}$ in accordance with the standard truncation scheme for these equations [5], where in the case of short-range interactions, power counting arguments establish the scheme's validity.

Since energy is conserved, the self-energy, the singleparticle Green's functions, the single-scale propagator and the vertex include the corresponding $\delta$ function,

$$
\begin{aligned}
\Sigma_{\alpha \beta}^{\Lambda}\left(\omega_{n} ; \omega_{n^{\prime}}\right) & \rightarrow T^{-1} \delta_{n, n^{\prime}} \Sigma_{\alpha \beta}^{\Lambda}\left(\omega_{n}\right) \\
\mathcal{G}_{\alpha \beta}^{0, \Lambda}\left(\omega_{n} ; \omega_{n^{\prime}}\right) & \rightarrow T^{-1} \delta_{n, n^{\prime}} \mathcal{G}_{\alpha \beta}^{0, \Lambda}\left(\omega_{n}\right) \\
\mathcal{G}_{\alpha \beta}^{\Lambda}\left(\omega_{n} ; \omega_{n^{\prime}}\right) & \rightarrow T^{-1} \delta_{n, n^{\prime}} \mathcal{G}_{\alpha \beta}^{\Lambda}\left(\omega_{n}\right), \\
\mathcal{S}_{\alpha \beta}^{\Lambda}\left(\omega_{n} ; \omega_{n^{\prime}}\right) & \rightarrow T^{-1} \delta_{n, n^{\prime}} \mathcal{S}_{\alpha \beta}^{\Lambda}\left(\omega_{n}\right), \\
\Gamma_{\alpha \beta \gamma \delta}^{\Lambda}\left(\omega_{n}, \omega_{\tilde{n}} ; \omega_{n^{\prime}}, \omega_{\tilde{n}^{\prime}}\right) & \rightarrow T^{-1} \delta_{n+\tilde{n}, n^{\prime}+\tilde{n}^{\prime}} \Gamma_{\alpha \beta \gamma \delta}^{\Lambda}\left(\omega_{n}, \omega_{\tilde{n}} ; \omega_{n^{\prime}}\right)
\end{aligned}
$$

Inserting this into Eq. (5) yields

$$
\begin{aligned}
& \frac{d}{d \Lambda} T^{-1} \delta_{n, n^{\prime}} \Sigma_{\alpha \beta}^{\Lambda}\left(\omega_{n}\right) \\
& =T^{2} \sum_{\omega_{m} \omega_{m^{\prime}}} \sum_{\mu \nu} \mathcal{S}_{\mu \nu}^{\Lambda}\left(\omega_{m}\right) \Gamma_{\alpha \nu \beta \mu}^{\Lambda}\left(\omega_{n}, \omega_{m^{\prime}} ; \omega_{n^{\prime}}\right) \\
& \quad \times T^{-1} \delta_{m, m^{\prime}} T^{-1} \delta_{n+m^{\prime}, n^{\prime}+m},
\end{aligned}
$$


and after evaluating the sum over the Matsubara frequency $\omega_{m^{\prime}}$, one arrives at

$$
\frac{d}{d \Lambda} \Sigma_{\alpha \beta}^{\Lambda}\left(\omega_{n}\right)=T \sum_{\omega_{m}} \sum_{\mu \nu} \mathcal{S}_{\mu \nu}^{\Lambda}\left(\omega_{m}\right) \Gamma_{\alpha \nu \beta \mu}^{\Lambda}\left(\omega_{n}, \omega_{m} ; \omega_{n}\right) .
$$

Here, we have used that a $\delta_{n, n^{\prime}}$ appears on both sides and have multiplied the equation by $T$.

Proceeding in a similar way for the equation of the flow of the vertex, Eq. (6), we arrive at

$$
\begin{aligned}
\frac{d}{d \Lambda} \Gamma_{\alpha \beta \gamma \delta}^{\Lambda}\left(\omega_{n}, \omega_{\tilde{n}} ; \omega_{n^{\prime}}\right)= & T \sum_{\omega_{m} \omega_{\tilde{m}}} \sum_{\mu \nu \rho \sigma} \mathcal{G}_{\rho \mu}^{\Lambda}\left(\omega_{m}\right) \mathcal{S}_{\sigma \nu}^{\Lambda}\left(\omega_{\tilde{m}}\right)\left\{\Gamma_{\alpha \beta \rho \sigma}^{\Lambda}\left(\omega_{n}, \omega_{\tilde{n}} ; \omega_{m}\right) \Gamma_{\mu \nu \gamma \delta}^{\Lambda}\left(\omega_{m}, \omega_{\tilde{m}} ; \omega_{n^{\prime}}\right) \delta_{\tilde{m}}^{(\mathrm{c})}\right. \\
& +\left[\Gamma_{\beta \nu \gamma \rho}^{\Lambda}\left(\omega_{\tilde{n}}, \omega_{\tilde{m}} ; \omega_{n^{\prime}}\right) \Gamma_{\mu \alpha \sigma \delta}^{\Lambda}\left(\omega_{m}, \omega_{n} ; \omega_{\tilde{m}}\right) \delta_{\tilde{m}}^{(\mathrm{ph}, 1)}+\Gamma_{\beta \mu \gamma \sigma}^{\Lambda}\left(\omega_{\tilde{n}}, \omega_{m} ; \omega_{n^{\prime}}\right) \Gamma_{\nu \alpha \rho \delta}^{\Lambda}\left(\omega_{\tilde{m}}, \omega_{n} ; \omega_{m}\right) \delta_{\tilde{m}}^{(\mathrm{ph}, 2)}\right] \\
& \left.-\left[\Gamma_{\alpha \nu \gamma \rho}^{\Lambda}\left(\omega_{n}, \omega_{\tilde{m}} ; \omega_{n^{\prime}}\right) \Gamma_{\mu \beta \sigma \delta}^{\Lambda}\left(\omega_{m}, \omega_{\tilde{n}} ; \omega_{\tilde{m}}\right) \delta_{\tilde{m}}^{(\mathrm{ph}, 3)}+\Gamma_{\alpha \mu \gamma \sigma}^{\Lambda}\left(\omega_{n}, \omega_{m} ; \omega_{n^{\prime}}\right) \Gamma_{\nu \beta \rho \delta}^{\Lambda}\left(\omega_{\tilde{m}}, \omega_{\tilde{m}} ; \omega_{m}\right) \delta_{\tilde{m}}^{(\mathrm{ph}, 4)}\right]\right\},(1)
\end{aligned}
$$

where $\delta_{\tilde{m}}^{(\mathrm{c})}$ and $\delta_{\tilde{m}}^{(\mathrm{ph}, \cdot)}$ reflect the energy conservation of the vertex, e.g., $\delta_{\tilde{m}}^{\mathrm{c}}=\delta_{n+\tilde{n}, m+\tilde{m}}$.

\section{B. Formalism at zero temperature}

For the most part, we will discuss the formalism at $T=0$. In that case, sums over Matsubara frequencies are replaced by integrals,

$$
T \sum_{\omega_{n}} \rightarrow(2 \pi)^{-1} \int d \omega
$$

and the Kronecker symbols will be replaced by $\delta$ functions,

$$
T^{-1} \delta_{n, n^{\prime}} \rightarrow 2 \pi \delta\left(\omega-\omega^{\prime}\right) .
$$

Equations (14) and (15) now read

$$
\begin{aligned}
& \frac{d}{d \Lambda} \Sigma_{\alpha \beta}^{\Lambda}(\omega)=\frac{1}{2 \pi} \int d \bar{\omega} \sum_{\mu \nu} \mathcal{S}_{\mu \nu}^{\Lambda}(\bar{\omega}) \Gamma_{\alpha \nu \beta \mu}^{\Lambda}(\omega, \bar{\omega} ; \omega), \\
& \frac{d}{d \Lambda} \Gamma_{\alpha \beta \gamma \delta}^{\Lambda}\left(\omega, \tilde{\omega} ; \omega^{\prime}\right)= \frac{1}{2 \pi} \int d \bar{\omega} d \bar{\omega}^{\prime} \sum_{\mu \nu \rho \sigma} \mathcal{G}_{\rho \mu}^{\Lambda}(\bar{\omega}) \mathcal{S}_{\sigma \nu}^{\Lambda}\left(\bar{\omega}^{\prime}\right) \times\left\{\Gamma_{\alpha \beta \rho \sigma}^{\Lambda}(\omega, \tilde{\omega} ; \bar{\omega}) \Gamma_{\mu \nu \gamma \delta}^{\Lambda}\left(\bar{\omega}, \bar{\omega}^{\prime} ; \omega^{\prime}\right) \delta^{(\mathrm{c})}\left(\bar{\omega}^{\prime}\right)\right. \\
&+\left[\Gamma_{\beta \nu \gamma \rho}^{\Lambda}\left(\tilde{\omega}, \bar{\omega}^{\prime} ; \omega^{\prime}\right) \Gamma_{\mu \alpha \sigma \delta}^{\Lambda}\left(\bar{\omega}, \omega ; \bar{\omega}^{\prime}\right) \delta^{(\mathrm{ph}, 1)}\left(\bar{\omega}^{\prime}\right)+\Gamma_{\beta \mu \gamma \sigma}^{\Lambda}\left(\tilde{\omega}, \bar{\omega} ; \omega^{\prime}\right) \Gamma_{\nu \alpha \rho \delta}^{\Lambda}\left(\bar{\omega}^{\prime}, \omega ; \bar{\omega}\right) \delta^{(\mathrm{ph}, 2)}\left(\bar{\omega}^{\prime}\right)\right] \\
&\left.-\left[\Gamma_{\alpha \nu \gamma \rho}^{\Lambda}\left(\omega, \bar{\omega}^{\prime} ; \omega^{\prime}\right) \Gamma_{\mu \beta \sigma \delta}^{\Lambda}\left(\bar{\omega}, \tilde{\omega} ; \bar{\omega}^{\prime}\right) \delta^{(\mathrm{ph}, 3)}\left(\bar{\omega}^{\prime}\right)+\Gamma_{\alpha \mu \gamma \sigma}^{\Lambda}\left(\omega, \bar{\omega} ; \omega^{\prime}\right) \Gamma_{\nu \beta \rho \delta}^{\Lambda}\left(\bar{\omega}^{\prime}, \tilde{\omega} ; \bar{\omega}\right) \delta^{(\mathrm{ph}, 4)}\left(\bar{\omega}^{\prime}\right)\right]\right\},
\end{aligned}
$$

where again, $\delta^{(\mathrm{c})}\left(\bar{\omega}^{\prime}\right)$ and $\delta^{(\mathrm{ph}, \cdot)}\left(\bar{\omega}^{\prime}\right)$ reflect the energy conservation of the vertex, e.g., $\delta^{(\mathrm{ph}, 1)}\left(\bar{\omega}^{\prime}\right)=\delta\left(\tilde{\omega}+\bar{\omega}^{\prime}-\omega^{\prime}-\bar{\omega}\right)$.

We now proceed to take the static limit, i.e., by replacing the frequency dependence of the vertex and the self-energy by their static limit, e.g., $\Gamma\left(\omega, \omega^{\prime} ; \bar{\omega}\right) \rightarrow \Gamma(0)$. For shortrange interactions, power counting of the flow equations demonstrates that the dominant contribution for small $\Lambda$ comes from zero frequencies and states close to the Fermi energy. This approximation has been discussed extensively in Ref. [5].

We arrive at

$$
\begin{aligned}
\frac{d}{d \Lambda} \Sigma_{\alpha \beta}^{\Lambda}= & \frac{1}{2 \pi} \int d \bar{\omega} \sum_{\mu \nu} \mathcal{S}_{\mu \nu}^{\Lambda}(\bar{\omega}) \Gamma_{\alpha \nu \beta \mu}^{\Lambda} \\
\frac{d}{d \Lambda} \Gamma_{\alpha \beta}^{\Lambda}= & \frac{1}{2 \pi} \int d \bar{\omega} \sum_{\mu \nu \rho \sigma}\left\{\mathcal{G}_{\rho \mu}^{\Lambda}(\bar{\omega}) \mathcal{S}_{\sigma \nu}^{\Lambda}(-\bar{\omega}) \Gamma_{\alpha \beta \rho \sigma}^{\Lambda} \Gamma_{\mu \nu \gamma \delta}^{\Lambda}\right. \\
& +\mathcal{G}_{\rho \mu}^{\Lambda}(\bar{\omega}) \mathcal{S}_{\sigma \nu}^{\Lambda}(\bar{\omega})\left[\Gamma_{\beta \nu \gamma \rho}^{\Lambda} \Gamma_{\mu \alpha \sigma \delta}^{\Lambda}+\Gamma_{\beta \mu \gamma \sigma}^{\Lambda} \Gamma_{\nu \alpha \rho \delta}^{\Lambda}\right] \\
& \left.-\mathcal{G}_{\rho \mu}^{\Lambda}(\bar{\omega}) \mathcal{S}_{\sigma \nu}^{\Lambda}(\bar{\omega})\left[\Gamma_{\alpha \nu \gamma \rho}^{\Lambda} \Gamma_{\mu \beta \sigma \delta}^{\Lambda}+\Gamma_{\alpha \mu \gamma \sigma}^{\Lambda} \Gamma_{\nu \beta \rho \delta}^{\Lambda}\right]\right\}
\end{aligned}
$$

Note that the vertex $\Gamma^{\Lambda}$ is antisymmetric under exchange of the first or the last pair of indices,

$$
\Gamma_{\alpha \beta \gamma \delta}^{\Lambda}=-\Gamma_{\beta \alpha \gamma \delta}^{\Lambda}=-\Gamma_{\alpha \beta \delta \gamma}^{\Lambda}=\Gamma_{\beta \alpha \delta \gamma}^{\Lambda} .
$$

Furthermore, one can easily show that in the static limit for finite system sizes the self-energy $\Sigma$ is Hermitian. To further simplify these equations, we choose our cutoff $\Theta^{\Lambda}(\omega)$ to be a simple step function,

$$
\Theta^{\Lambda}(\omega)=\Theta(|\omega|-\Lambda),
$$

such that its derivative is

$$
\frac{d}{d \Lambda} \Theta^{\Lambda}(\omega)=-\delta(|\omega|-\Lambda)
$$

Since by construction the self-energy is not frequency dependent, the frequency integrals may now be solved analytically. For Eq. (20), we have to integrate

$$
\int d \bar{\omega} \mathcal{S}_{\mu \nu}^{\Lambda}(\bar{\omega})
$$


Inserting Dyson's equation into Eq. (7), we have

$$
\begin{aligned}
\mathcal{S} & =-\mathcal{G}\left(\frac{d}{d \Lambda}\left[\mathcal{G}^{0}\right]^{-1}\right) \mathcal{G}=-\mathcal{G}\left(\frac{d}{d \Lambda}\left[\mathcal{G}^{-1}+\Sigma\right]\right) \mathcal{G} \\
& =\dot{\mathcal{G}}-\mathcal{G} \dot{\Sigma} \mathcal{G},
\end{aligned}
$$

in matrix notation. We note that $\mathcal{G}=(\mathcal{Q}-\Theta \Sigma)^{-1} \Theta$, where we use the shorthand $\Theta=\Theta(|\omega|-\Lambda)$ and $\mathcal{Q}=\mathrm{i} \omega-H_{0}+$ $\mu_{\text {chem. }}$. Using $\frac{d}{d \Lambda} A^{-1}(\Lambda)=-A^{-1}(\Lambda) \dot{A}(\Lambda) A^{-1}(\Lambda)$, simple algebra yields

$$
\mathcal{S}=-\delta\left(\mathbb{1}+\frac{\Theta}{\mathcal{Q}-\Theta \Sigma} \Sigma\right) \frac{1}{\mathcal{Q}-\Theta \Sigma} .
$$

Since the $\delta$ and $\Theta$ functions are to be taken at the same argument, we employ Morris's Lemma ${ }^{3}$ to resolve this,

$$
\mathcal{S}=-\delta \int_{0}^{1} d t\left(\mathbb{1}+t \frac{1}{\mathcal{Q}-t \Sigma} \Sigma\right) \frac{1}{\mathcal{Q}-t \Sigma} .
$$

Using the fact that

$$
\frac{d}{d t} \frac{1}{\mathcal{Q}-t \Sigma}=\frac{1}{\mathcal{Q}-t \Sigma} \Sigma \frac{1}{\mathcal{Q}-t \Sigma}
$$

and partial integration, the second summand of the integral yields

$$
-\left[\frac{t}{\mathcal{Q}-t \Sigma}\right]_{0}^{1}+\int_{0}^{1} d t \frac{1}{\mathcal{Q}-t \Sigma},
$$

where it can be seen that the remaining integral cancels the first summand of the integral in Eq. (27), so we arrive at

$$
\mathcal{S}^{\Lambda}(\omega)=-\frac{\delta(|\omega|-\Lambda)}{\mathrm{i} \omega-H_{0}+\mu_{\text {chem }}-\Sigma^{\Lambda}} .
$$

The frequency integral is now trivial, yielding

$$
\int d \bar{\omega} \mathcal{S}^{\Lambda}(\bar{\omega})=-\sum_{\bar{\omega}= \pm \Lambda} \frac{1}{\mathrm{i} \bar{\omega}-H_{0}+\mu_{\mathrm{chem}}-\Sigma^{\Lambda}} .
$$

As the following quantity will appear also in the flow equation for the vertex, we will define

$$
P_{\mu \nu}^{\Lambda}(\bar{\omega}):=\left.\frac{1}{\mathrm{i} \bar{\omega}-H_{0}+\mu_{\mathrm{chem}}-\Sigma^{\Lambda}}\right|_{\mu \nu} .
$$

Inserting Eqs. (29) and (30) into Eq. (20), the flow equation for the self-energy now reads

$$
\frac{d}{d \Lambda} \Sigma_{\alpha \beta}^{\Lambda}=-\frac{1}{2 \pi} \sum_{\mu \nu} \underbrace{\left(P_{\mu \nu}^{\Lambda}(\Lambda)+P_{\mu \nu}^{\Lambda}(-\Lambda)\right)}_{=: \Pi_{\mu \nu}^{\Sigma, \Lambda}} \Gamma_{\alpha \nu \beta \mu}^{\Lambda} .
$$

When evaluating the flow equation for the vertex, Eq. (21), one must take care that the arguments for the $\delta$ and $\Theta$ functions coincide, so one may not simply take the result derived for the single-scale propagator in the self-energy flow and apply it, but one rather uses the same kind of treatment of the $\delta$ and $\Theta$ functions for the entire expression, on a term by term basis.

\footnotetext{
${ }^{3} \delta(x) f(\Theta(x)) \rightarrow \delta(x) \int_{0}^{1} f(t) d t$, see Ref. [95].
}

In the end, the flow equation for the vertex in the static limit reads,

$$
\begin{aligned}
\frac{d}{d \Lambda} \Gamma_{\alpha \beta \gamma \delta}^{\Lambda}= & -\frac{1}{2 \pi} \sum_{\mu \nu \rho \sigma} \sum_{\bar{\omega}= \pm \Lambda}\left\{\frac{1}{2} P_{\rho \mu}^{\Lambda}(-\bar{\omega}) P_{\sigma \nu}^{\Lambda}(\bar{\omega}) \Gamma_{\alpha \beta \rho \sigma}^{\Lambda} \Gamma_{\mu \nu \gamma \delta}^{\Lambda}\right. \\
& \left.+P_{\rho \mu}^{\Lambda}(\bar{\omega}) P_{\sigma \nu}^{\Lambda}(\bar{\omega})\left[\Gamma_{\beta \nu \gamma \rho}^{\Lambda} \Gamma_{\alpha \mu \delta \sigma}^{\Lambda}-\Gamma_{\alpha \nu \gamma \rho}^{\Lambda} \Gamma_{\beta \mu \delta \sigma}^{\Lambda}\right]\right\} \\
= & -\frac{1}{2 \pi} \sum_{\mu \nu \rho \sigma}\left\{\Pi_{\mu \nu \sigma \rho}^{\mathrm{c}, \Lambda} \Gamma_{\nu \rho \gamma \delta}^{\Lambda} \Gamma_{\alpha \beta \sigma \mu}^{\Lambda}\right. \\
& \left.+\Pi_{\mu \nu \rho \sigma \sigma}^{\mathrm{ph}, \Lambda}\left[\Gamma_{\beta \nu \gamma \rho}^{\Lambda} \Gamma_{\alpha \sigma \delta \mu}^{\Lambda}-\Gamma_{\alpha \nu \gamma \rho}^{\Lambda} \Gamma_{\beta \sigma \delta \mu}^{\Lambda}\right]\right\},
\end{aligned}
$$

where we have used the symmetries of $\Gamma$ to simplify the equations and abbreviated

$$
\begin{aligned}
& \Pi_{\mu \nu \sigma \rho}^{\mathrm{c}, \Lambda}:=P_{\mu \nu}^{\Lambda}(\Lambda) P_{\sigma \rho}^{\Lambda}(-\Lambda), \\
& \Pi_{\mu \nu \sigma \rho}^{\mathrm{ph}, \Lambda}:=P_{\mu \nu}^{\Lambda}(\Lambda) P_{\rho \sigma}^{\Lambda}(\Lambda)+P_{\mu \nu}^{\Lambda}(-\Lambda) P_{\rho \sigma}^{\Lambda}(-\Lambda) .
\end{aligned}
$$

The full derivation may be found in Appendix A.

\section{Initial conditions}

The initial conditions at $\Lambda \rightarrow \infty$ are given by

$$
\Sigma_{\alpha \beta}^{\Lambda \rightarrow \infty}=0 \quad \text { and } \quad \Gamma_{\alpha \beta \gamma \delta}^{\Lambda \rightarrow \infty}=U_{\alpha \beta \gamma \delta} .
$$

In order to solve the equations numerically, we need to choose an initial value $\Lambda_{0}$ that is still finite but larger than all other energy scales in the system. For $\Lambda>\Lambda_{0}$, one may assume a form of $(i \omega)^{-1} \mathbb{1}$ for the propagator, allowing us to analytically integrate the flow equations from $\infty$ to $\Lambda_{0}$. In case of the flow equation for the vertex, power counting in $U$ and $\Lambda_{0}$ immediately yields

$$
\Gamma^{\Lambda_{0}}-U \sim-\int_{\infty}^{\Lambda_{0}} U U \frac{1}{\Lambda^{2}} d \Lambda=\frac{1}{\Lambda_{0}} U U,
$$

and hence

$$
\left|\Gamma^{\Lambda_{0}}-U\right| /|U| \sim|U| / \Lambda_{0} .
$$

We therefore may simply use that $\Gamma^{\Lambda_{0}}$ does not differ from $\Gamma^{\Lambda \rightarrow \infty}$ for large enough $\Lambda_{0}$ and arrive at

$$
\Gamma_{\alpha \beta \gamma \delta}^{\Lambda}=U_{\alpha \beta \gamma \delta}, \quad \Lambda>\Lambda_{0} .
$$

The same does not hold true for the flow equation for the selfenergy, where the analytical integral gives a non-negligible contribution,

$$
\begin{aligned}
\Sigma_{\alpha \beta}^{\Lambda_{0}} & =-\frac{1}{2 \pi} \sum_{\mu} U_{\alpha \mu \beta \mu} \lim _{\eta \rightarrow 0^{+}} \int_{\infty}^{\Lambda_{0}}\left(\frac{\mathrm{e}^{\mathrm{i} \Lambda \eta}}{\mathrm{i} \Lambda}-\frac{\mathrm{e}^{-\mathrm{i} \Lambda \eta}}{\mathrm{i} \Lambda}\right) d \Lambda \\
& =-\frac{1}{\pi} \sum_{\mu} U_{\alpha \mu \beta \mu} \lim _{\eta \rightarrow 0^{+}} \eta \int_{\infty}^{\Lambda_{0}} \operatorname{sinc}(\eta \Lambda) d \Lambda \\
& =\frac{1}{\pi} \sum_{\mu} U_{\alpha \mu \beta \mu} \lim _{\eta \rightarrow 0^{+}}\left[\int_{0}^{\infty} \operatorname{sinc}(x) d x-\mathcal{O}(\eta)\right] \\
& =\frac{1}{2} \sum_{\mu} U_{\alpha \mu \beta \mu} .
\end{aligned}
$$

Here we have explicitly included the required convergence factor $\mathrm{e}^{\mathrm{i} \omega 0^{+}}$that appears in the Green's function in imaginary frequency space. 


\section{Systems with spin}

In Eqs. (31) and (32), the indices represent generic states in the Hilbert space. We will now discuss the case where the system is fully $\mathrm{SU}(2)$ symmetric. Here, it is convenient to separate the orbital degrees of freedom from the spin degrees of freedom, $\alpha \rightarrow\left(\alpha, \sigma_{1}\right)$. Our derivation will follow Ref. [72], but we will discuss the generic case without the additional particlehole symmetry. Single-particle quantities (self-energy, propagators) do not depend on the spin degree of freedom,

$$
\begin{aligned}
& \Sigma_{\left(\alpha, \sigma_{1}\right)\left(\beta, \sigma_{2}\right)}^{\Lambda}=\Sigma_{\alpha \beta}^{\mathrm{s}, \Lambda} \delta_{\sigma_{1} \sigma_{2}}, \\
& \mathcal{G}_{\left(\alpha, \sigma_{1}\right)\left(\beta, \sigma_{2}\right)}^{\Lambda}=\mathcal{G}_{\alpha \beta}^{\mathrm{s}, \Lambda} \delta_{\sigma_{1} \sigma_{2}}, \\
& \mathcal{S}_{\left(\alpha, \sigma_{1}\right)\left(\beta, \sigma_{2}\right)}^{\Lambda}=\mathcal{S}_{\alpha \beta}^{\mathrm{s}, \Lambda} \delta_{\sigma_{1} \sigma_{2}}, \\
& P_{\left(\alpha, \sigma_{1}\right)\left(\beta, \sigma_{2}\right)}^{\Lambda}=P_{\alpha \beta}^{\mathrm{s}, \Lambda} \delta_{\sigma_{1} \sigma_{2}} .
\end{aligned}
$$

The spin structure of the vertex is determined by the fact that two particles may either keep their spin or exchange it, and may thus be decomposed into

$$
\Gamma_{\left(\alpha, \sigma_{1}\right),\left(\beta, \sigma_{2}\right),\left(\gamma, \sigma_{3}\right),\left(\delta, \sigma_{4}\right)}^{\Lambda}=c_{\alpha \beta \gamma \delta}^{\mathrm{I}, \Lambda} \delta_{\sigma_{1} \sigma_{3}} \delta_{\sigma_{2} \sigma_{4}}+c_{\alpha \beta \gamma \delta}^{\mathrm{II}, \Lambda} \delta_{\sigma_{1} \sigma_{4}} \delta_{\sigma_{2} \sigma_{3}},
$$

where $c^{\mathrm{I}}$ and $c^{\mathrm{II}}$ are the coefficients for each of these processes.

Using the antisymmetry of $\Gamma$, Eq. (22), we may exchange $\left(\gamma, \sigma_{3}\right)$ with $\left(\delta, \sigma_{4}\right)$,

$$
\begin{aligned}
\Gamma_{\left(\alpha, \sigma_{1}\right),\left(\beta, \sigma_{2}\right),\left(\gamma, \sigma_{3}\right),\left(\delta, \sigma_{4}\right)}^{\Lambda} & =-\Gamma_{\left(\alpha, \sigma_{1}\right),\left(\beta, \sigma_{2}\right),\left(\delta, \sigma_{4}\right),\left(\gamma, \sigma_{3}\right)}^{\Lambda} \\
& =-c_{\alpha \beta \delta \gamma}^{\mathrm{I}, \Lambda} \delta_{\sigma_{1} \sigma_{4}} \delta_{\sigma_{2} \sigma_{3}}-c_{\alpha \beta \delta \gamma}^{\mathrm{II}, \Lambda} \delta_{\sigma_{1} \sigma_{3}} \delta_{\sigma_{2} \sigma_{4}} .
\end{aligned}
$$

By comparing the coefficients of the Kronecker $\delta$ s, we may identify

$$
c_{\alpha \beta \gamma \delta}^{\mathrm{I}, \Lambda}=-c_{\alpha \beta \delta \gamma}^{\mathrm{II}, \Lambda}:=-\Gamma_{\alpha \beta \delta \gamma}^{\mathrm{s}, \Lambda},
$$

and hence write the vertex as

$$
\Gamma_{\left(\alpha, \sigma_{1}\right),\left(\beta, \sigma_{2}\right),\left(\gamma, \sigma_{3}\right),\left(\delta, \sigma_{4}\right)}^{\Lambda}=\Gamma_{\alpha \beta \gamma \delta}^{\mathrm{s}, \Lambda} \delta_{\sigma_{1} \sigma_{4}} \delta_{\sigma_{2} \sigma_{3}}-\Gamma_{\alpha \beta \delta \gamma}^{\mathrm{s}, \Lambda} \delta_{\sigma_{1} \sigma_{3}} \delta_{\sigma_{2} \sigma_{4}} .
$$

Using the symmetry of $\Gamma^{\Lambda}$, one can see that $\Gamma^{\mathrm{s}, \Lambda}$ is still symmetric under exchange of both pairs of indices,

$$
\Gamma_{\alpha \beta \gamma \delta}^{\mathrm{s}, \Lambda}=\Gamma_{\beta \alpha \delta \gamma}^{\mathrm{s}, \Lambda},
$$

but in general it is not antisymmetric with respect to the exchange of a single pair of indices. Instead, one may identify the part of $\Gamma^{\mathrm{s}, \Lambda}$ that is antisymmetric under exchange of $\alpha$ and $\beta$ with the triplet channel of the vertex, whereas the part that is symmetric under the exchange of $\alpha$ and $\beta$ represents the singlet channel.

Inserting Eqs. (40), (43), and (44) into Eq. (31), we have

$$
\begin{aligned}
\frac{d}{d \Lambda} \Sigma_{\alpha \beta}^{\mathrm{s}, \Lambda} \delta_{\sigma_{1} \sigma_{2}}= & -\frac{1}{2 \pi} \sum_{\mu \nu} \sum_{\sigma_{3}} \Pi_{\mu \nu}^{\Sigma, \mathrm{s}, \Lambda}\left(\Gamma_{\alpha \nu \beta \mu}^{\mathrm{s}, \Lambda} \delta_{\sigma_{1} \sigma_{3}} \delta_{\sigma_{3} \sigma_{2}}\right. \\
& \left.-\Gamma_{\alpha \nu \mu \beta}^{\mathrm{s}, \Lambda} \delta_{\sigma_{1} \sigma_{2}} \delta_{\sigma_{3} \sigma_{3}}\right) \\
= & -\frac{1}{2 \pi} \sum_{\mu \nu} \Pi_{\mu \nu}^{\Sigma, \mathrm{s}, \Lambda}\left(\Gamma_{\alpha \nu \beta \mu}^{\mathrm{s}, \Lambda}-2 \Gamma_{\alpha \nu \mu \beta}^{\mathrm{s}, \Lambda}\right) \delta_{\sigma_{1} \sigma_{2}},
\end{aligned}
$$

and hence

$$
\frac{d}{d \Lambda} \Sigma_{\alpha \beta}^{\mathrm{s}, \Lambda}=-\frac{1}{2 \pi} \sum_{\mu \nu} \Pi_{\mu \nu}^{\Sigma, \mathrm{s}, \Lambda}\left(\Gamma_{\alpha \nu \beta \mu}^{\mathrm{s}, \Lambda}-2 \Gamma_{\alpha \nu \mu \beta}^{\mathrm{s}, \Lambda}\right) .
$$

Here, we have defined

$$
\Pi_{\mu \nu}^{\Sigma, \mathrm{s}, \Lambda}:=P_{\mu \nu}^{\mathrm{s}, \Lambda}(\Lambda)+P_{\mu \nu}^{\mathrm{s}, \Lambda}(-\Lambda)
$$

in analogy to the definition in Eq. (31), as we will do with $\Pi_{\mu \nu}^{\mathrm{ph}, \mathrm{s}, \Lambda}$ and $\Pi_{\mu \nu}^{\mathrm{c}, \mathrm{s}, \Lambda}$ in the following. To obtain the flow equation for $\Gamma^{\Lambda, s}$, we must insert Eqs. (40), (43), and (44) into Eq. (32). To simplify our notation, we will use $\delta_{12}^{34}=\delta_{\sigma_{1} \sigma_{2}} \delta_{\sigma_{3} \sigma_{4}}$. For the first term with $\Pi^{\mathrm{c}, \mathrm{s}, \Lambda}$, we have

$$
\begin{aligned}
& -\frac{1}{2 \pi} \sum_{\mu \nu \rho \sigma} \sum_{\sigma_{5} \sigma_{6}} \Pi_{\mu \nu \sigma \rho}^{\mathrm{c}, \mathrm{s}, \Lambda}\left(\Gamma_{\nu \rho \gamma \delta}^{\mathrm{s}, \Lambda} \delta_{54}^{63}-\Gamma_{\nu \rho \delta \gamma}^{\mathrm{s}, \Lambda} \delta_{53}^{64}\right) \\
& \quad \times\left(\Gamma_{\alpha \beta \sigma \mu}^{\mathrm{s}, \Lambda} \delta_{15}^{26}-\Gamma_{\alpha \beta \mu \sigma}^{\mathrm{s}, \Lambda} \delta_{16}^{25}\right) .
\end{aligned}
$$

Multiplying out the main product, there are four terms of combinations of $\Gamma^{\mathrm{s}, \Lambda}$ that appear:

$$
\begin{aligned}
& \sum_{\sigma_{5} \sigma_{6}} \Gamma_{v \rho \gamma \delta}^{\mathrm{s}, \Lambda} \Gamma_{\alpha \beta \sigma \mu}^{\mathrm{s}, \Lambda} \delta_{54}^{63} \delta_{15}^{26}=\Gamma_{v \rho \gamma \delta}^{\mathrm{s}, \Lambda} \Gamma_{\alpha \beta \sigma \mu}^{\mathrm{s}, \Lambda} \delta_{14}^{23}, \\
& \sum_{\sigma_{5} \sigma_{6}}-\Gamma_{\nu \rho \delta \gamma}^{\mathrm{s}, \Lambda} \Gamma_{\alpha \beta \sigma \mu}^{\mathrm{s}, \Lambda} \delta_{53}^{64} \delta_{15}^{26}=-\Gamma_{\nu \rho \delta \gamma}^{\mathrm{s}, \Lambda} \Gamma_{\alpha \beta \sigma \mu}^{\mathrm{s}, \Lambda} \delta_{13}^{24}, \\
& \sum_{\sigma_{5} \sigma_{6}}-\Gamma_{\nu \rho \gamma \delta}^{\mathrm{s}, \Lambda} \Gamma_{\alpha \beta \mu \sigma}^{\mathrm{s}, \Lambda} \delta_{54}^{63} \delta_{16}^{25}=-\Gamma_{\nu \rho \gamma \delta}^{\mathrm{s}, \Lambda} \Gamma_{\alpha \beta \mu \sigma}^{\mathrm{s}, \Lambda} \delta_{13}^{24}, \\
& \sum_{\sigma_{5} \sigma_{6}} \Gamma_{\nu \rho \delta \gamma}^{\mathrm{s}, \Lambda} \Gamma_{\alpha \beta \mu \sigma}^{\mathrm{s}, \Lambda} \delta_{53}^{64} \delta_{16}^{25}=\Gamma_{\nu \rho \delta \gamma}^{\mathrm{s}, \Lambda} \Gamma_{\alpha \beta \mu \sigma}^{\mathrm{s}, \Lambda} \delta_{14}^{23} .
\end{aligned}
$$

On the other hand, the left-hand side of the flow equation reads

$$
\frac{d}{d \Lambda}\left(\Gamma_{\alpha \beta \gamma \delta}^{\mathrm{s}, \Lambda} \delta_{14}^{23}-\Gamma_{\alpha \beta \delta \gamma}^{\mathrm{s}, \Lambda} \delta_{13}^{24}\right) .
$$

We may thus look at the products that contain $\delta_{14}^{23}$ to obtain the first term of the flow equation for $\Gamma^{\mathrm{s}, \Lambda}$,

$$
-\frac{1}{2 \pi} \sum_{\mu \nu \rho \sigma} \Pi_{\mu \nu \sigma \rho}^{\mathrm{c}, \Lambda}\left(\Gamma_{\nu \rho \gamma \delta}^{\mathrm{s}, \Lambda} \Gamma_{\alpha \beta \sigma \mu}^{\mathrm{s}, \Lambda}+\Gamma_{\nu \rho \delta \gamma}^{\mathrm{s}, \Lambda} \Gamma_{\alpha \beta \mu \sigma}^{\mathrm{s}, \Lambda}\right) \delta_{14}^{23} .
$$

We may now proceed in doing the same for the particle-hole channel,

$$
\begin{aligned}
& -\frac{1}{2 \pi} \sum_{\mu \nu \rho \sigma} \sum_{\sigma_{5} \sigma_{6}} \Pi_{\mu \nu \rho \sigma}^{\mathrm{ph}, \mathrm{s}, \Lambda} \\
& \quad \times\left\{\left(\Gamma_{\alpha \nu \gamma \rho}^{\mathrm{s}, \Lambda} \delta_{53}^{16}-\Gamma_{\alpha \nu \rho \gamma}^{\mathrm{s}, \Lambda} \delta_{56}^{13}\right)\left(\Gamma_{\beta \sigma \delta \mu}^{\mathrm{s}, \Lambda} \delta_{64}^{25}-\Gamma_{\beta \sigma \mu \delta}^{\mathrm{s}, \Lambda} \delta_{65}^{24}\right)\right. \\
& \left.\quad+\left(\Gamma_{\beta \nu \gamma \rho}^{\mathrm{s}, \Lambda} \delta_{53}^{26}-\Gamma_{\beta \nu \rho \gamma}^{\mathrm{s}, \Lambda} \delta_{56}^{23}\right)\left(\Gamma_{\alpha \sigma \delta \mu}^{\mathrm{s}, \Lambda} \delta_{64}^{15}-\Gamma_{\alpha \sigma \mu \delta}^{\mathrm{s}, \Lambda} \delta_{65}^{14}\right)\right\} .
\end{aligned}
$$

Of the eight products that appear, we again pick out those that appear with a $\delta_{14}^{23}$, where we use that

$$
\begin{array}{ll}
\sum_{\sigma_{5} \sigma_{6}} \delta_{53}^{16} \delta_{64}^{25}=\delta_{14}^{23}, & \sum_{\sigma_{5} \sigma_{6}} \delta_{56}^{23} \delta_{65}^{14}=2 \delta_{14}^{23}, \\
\sum_{\sigma_{5} \sigma_{6}} \delta_{53}^{26} \delta_{65}^{14}=\delta_{14}^{23}, & \sum_{\sigma_{5} \sigma_{6}} \delta_{56}^{23} \delta_{64}^{15}=\delta_{14}^{23},
\end{array}
$$

so that we arrive at

$$
\begin{aligned}
& -\frac{1}{2 \pi} \sum_{\mu \nu \rho \sigma} \Pi_{\mu \nu \rho \sigma}^{\mathrm{ph}, \mathrm{s}, \Lambda}\left(2 \Gamma_{\beta \nu \rho \gamma}^{\mathrm{s}, \Lambda} \Gamma_{\alpha \sigma \mu \delta}^{\mathrm{s}, \Lambda}+\Gamma_{\alpha \nu \gamma \rho}^{\mathrm{s}, \Lambda} \Gamma_{\beta \sigma \delta \mu}^{\mathrm{s}, \Lambda}\right. \\
& \left.-\Gamma_{\beta \nu \rho \gamma}^{\mathrm{s}, \Lambda} \Gamma_{\alpha \sigma \delta \mu}^{\mathrm{s}, \Lambda}-\Gamma_{\beta \nu \gamma \rho}^{\mathrm{s}, \Lambda} \Gamma_{\alpha \sigma \mu \delta}^{\mathrm{s}, \Lambda}\right) \delta_{14}^{23} .
\end{aligned}
$$


Adding Eqs. (50) and (52), the flow equation for $\Gamma^{\mathrm{s}, \Lambda}$ now reads

$$
\begin{aligned}
\frac{d}{d \Lambda} \Gamma_{\alpha \beta \delta \gamma}^{\mathrm{s}, \Lambda}= & -\frac{1}{2 \pi} \sum_{\mu \nu \rho \sigma}\left\{\Pi_{\mu \nu \sigma \rho}^{\mathrm{c}, \mathrm{s}, \Lambda}\left(\Gamma_{\nu \rho \gamma \delta}^{\mathrm{s}, \Lambda} \Gamma_{\alpha \beta \sigma \mu}^{\mathrm{s}, \Lambda}+\Gamma_{\nu \rho \delta \gamma}^{\mathrm{s}, \Lambda} \Gamma_{\alpha \beta \mu \sigma}^{\mathrm{s}, \Lambda}\right)\right. \\
& +\Pi_{\mu \nu \rho \sigma}^{\mathrm{ph}, \mathrm{s}, \Lambda}\left(2 \Gamma_{\beta \nu \rho \gamma}^{\mathrm{s}, \Lambda} \Gamma_{\alpha \sigma \mu \delta}^{\mathrm{s}, \Lambda}+\Gamma_{\alpha \nu \gamma \rho}^{\mathrm{s}, \Lambda} \Gamma_{\beta \sigma \delta \mu}^{\mathrm{s}, \Lambda}\right. \\
& \left.\left.-\Gamma_{\beta \nu \rho \gamma}^{\mathrm{s}, \Lambda} \Gamma_{\alpha \sigma \delta \mu}^{\mathrm{s}, \Lambda}-\Gamma_{\beta \nu \gamma \rho}^{\mathrm{s}, \Lambda} \Gamma_{\alpha \sigma \mu \delta}^{\mathrm{s}, \Lambda}\right)\right\}
\end{aligned}
$$

\section{Finite temperature}

For completeness, we also derive the form of the flow equations at finite temperature. Thereby, the effect of Fermisurface smearing will be accounted for. Since the vertex function and the self-energy continue to be energy-independent, dynamical effects that describe fluctuations of thermal energies and entropy are not accounted for in this approach. Hence dephasing related phenomena like the many-body localization are expected to escape this treatment.

For the finite-temperature extension, we utilize the cutoff suggested in Ref. [73], hence we replace $\Theta(|\omega|-\Lambda)$ by $\chi^{\Lambda}\left(\omega_{n}\right)$, which is given by

$$
\chi^{\Lambda}\left(\omega_{n}\right)=\left\{\begin{array}{lll}
0, & \left|\omega_{n}\right| & \leqslant \Lambda-\pi T, \\
\frac{1}{2}+\frac{\left|\omega_{n}\right|-\Lambda}{2 \pi T} & \Lambda-\pi T \leqslant\left|\omega_{n}\right| \leqslant \Lambda+\pi T, \\
1, & \Lambda+\pi T \leqslant\left|\omega_{n}\right|,
\end{array}\right.
$$

and its derivative with respect to $\Lambda$ is then given by

$$
-\left(\partial_{\Lambda} \chi^{\Lambda}\left(\omega_{n}\right)\right)= \begin{cases}\frac{1}{2 \pi T} & \Lambda-\pi T \leqslant\left|\omega_{n}\right| \leqslant \Lambda+\pi T, \\ 0 & \text { otherwise. }\end{cases}
$$

We note that $\chi^{\Lambda}\left(\omega_{n}\right) \rightarrow \Theta(|\omega|-\Lambda)$ as $T \rightarrow 0$. The full Green's function is now given by

$$
\mathcal{G}^{\Lambda}\left(\omega_{n}\right)=\frac{\chi^{\Lambda}\left(\omega_{n}\right)}{\mathrm{i} \omega_{n}-H_{0}+\mu_{\text {chem }}-\chi^{\Lambda}\left(\omega_{n}\right) \Sigma^{\Lambda}\left(\omega_{n}\right)},
$$

whereas the single-scale propagator, Eq. (7), reads

$$
\begin{aligned}
\mathcal{S}^{\Lambda}\left(\omega_{n}\right)= & \frac{\partial_{\Lambda} \chi^{\Lambda}\left(\omega_{n}\right)}{\mathrm{i} \omega_{n}-H_{0}+\mu_{\text {chem }}-\chi^{\Lambda}\left(\omega_{n}\right) \Sigma^{\Lambda}\left(\omega_{n}\right)} \\
& \times\left(\mathrm{i} \omega_{n}-H_{0}+\mu_{\text {chem }}\right) \\
& \times \frac{1}{\mathrm{i} \omega_{n}-H_{0}+\mu_{\text {chem }}-\chi^{\Lambda}\left(\omega_{n}\right) \Sigma^{\Lambda}\left(\omega_{n}\right)} .
\end{aligned}
$$

With this form of a cutoff function, the Matsubara sums may be evaluated analytically. Since Matsubara frequencies have a distance of $2 \pi T$ from each other, the derivative of the cutoff is only nonzero for a two Matsubara frequencies, whose magnitude are that closest to the parameter $\Lambda$. Any sum with a single derivative of $\chi^{\Lambda}$ may hence be evaluated as

$$
T \sum_{n}-\left(\partial_{\Lambda} \chi^{\Lambda}\left(\omega_{n}\right)\right) f\left(\omega_{n}\right)=\frac{1}{2 \pi} \sum_{\left|\omega_{n}\right| \approx \Lambda} f\left(\omega_{n}\right) .
$$

This structure is very similar to the situation at $T=0$, where we have

$$
\frac{1}{2 \pi} \int d \omega \delta(|\omega|-\Lambda) f(\omega)=\frac{1}{2 \pi} \sum_{|\omega|=\Lambda} f(\omega) .
$$

Again we adopt the static limit and define $P^{T, \Lambda}\left(\omega_{n}\right)$ as

$$
P^{T, \Lambda}\left(\omega_{n}\right):=\frac{1}{\mathrm{i} \omega_{n}-H_{0}+\mu_{\mathrm{chem}}-\chi^{\Lambda}\left(\omega_{n}\right) \Sigma^{\Lambda}},
$$

and $P^{\prime T, \Lambda}, P^{\prime \prime} T, \Lambda$ as

$$
\begin{aligned}
& P^{\prime T, \Lambda}\left(\omega_{n}\right):=P^{T, \Lambda}\left(\omega_{n}\right)\left(\mathrm{i} \omega_{n}-H_{0}+\mu_{\mathrm{chem}}\right) P^{T, \Lambda}\left(\omega_{n}\right), \\
& P^{\prime \prime T, \Lambda}\left(\omega_{n}\right):=P^{T, \Lambda}\left(\omega_{n}\right) \chi^{\Lambda}\left(\omega_{n}\right),
\end{aligned}
$$

the flow equation for the self-energy now reads

$$
\frac{d}{d \Lambda} \Sigma_{\alpha \beta}^{\Lambda}=-\frac{1}{2 \pi} \sum_{\left|\omega_{n}\right| \approx \Lambda} P_{\mu \nu}^{\prime T, \Lambda}\left(\omega_{n}\right) \Gamma_{\alpha \nu \beta \mu}^{\Lambda} .
$$

Setting all external frequencies to zero and dropping the frequency dependence of the vertex, its flow equation is now given by

$$
\begin{aligned}
\frac{d}{d \Lambda} \Gamma_{\alpha \beta \gamma \delta}^{\Lambda}= & -\frac{1}{2 \pi} \sum_{\left|\omega_{n}\right| \approx \Lambda} \sum_{\mu \nu \rho \sigma}\left\{P_{\mu \nu}^{\prime \prime T, \Lambda}\left(\omega_{n}\right) P_{\rho \sigma}^{\prime T, \Lambda}\left(-\omega_{n}\right)\right. \\
& \times \Gamma_{\alpha \beta \sigma \mu}^{\Lambda} \Gamma_{\nu \rho \gamma \delta}^{\Lambda}+P_{\mu \nu}^{\prime \prime T, \Lambda}\left(\omega_{n}\right) P_{\rho \sigma}^{\prime T, \Lambda}\left(\omega_{n}\right) \\
& \times\left[\Gamma_{\beta \nu \gamma \rho}^{\Lambda} \Gamma_{\alpha \mu \delta \sigma}^{\Lambda}-\Gamma_{\alpha \mu \gamma \sigma}^{\Lambda} \Gamma_{\beta \nu \delta \rho}^{\Lambda}\right. \\
& \left.\left.+\Gamma_{\beta \mu \gamma \sigma}^{\Lambda} \Gamma_{\alpha \nu \delta \rho}^{\Lambda}-\Gamma_{\alpha \nu \gamma \rho}^{\Lambda} \Gamma_{\beta \mu \delta \sigma}^{\Lambda}\right]\right\}
\end{aligned}
$$

We note that $\chi^{\Lambda}\left(\omega_{n}\right) \rightarrow \frac{1}{2}$ for $\omega_{n} \rightarrow \Lambda$, so if taking the limit $T \rightarrow 0$ (and applying the symmetries of the vertex) one recovers Eq. (32).

\section{E. Observables and correlators}

\section{Single-particle observables}

Single-particle observables may be expressed by the Green's function, which is given by

$$
\mathcal{G}(\mathrm{i} \omega)=\frac{1}{\mathrm{i} \omega-H_{0}+\mu-\Sigma} \mathrm{e}^{\mathrm{i} \omega 0^{+}} .
$$

The convergence factor $\mathrm{e}^{\mathrm{i} \omega 0^{+}}$is explicitly required here. In the following, we will summarize (trivial) statements that follow from employing the static limit. For example, the density matrix for the occupancy of single-particle states, $\rho_{i j}$, is given by

$$
\rho_{i j}=\sum_{\alpha \beta} V_{i \alpha}^{\mathrm{rn}}\left[\frac{1}{2 \pi} \int_{-\infty}^{\infty} d \omega \mathcal{G}_{\alpha \beta}(\mathrm{i} \omega) \mathrm{e}^{\mathrm{i} \omega 0^{+}}\right] V_{\beta j}^{\mathrm{rn},-1},
$$

where

$$
V_{i \alpha}^{\mathrm{rn}}=\langle\alpha \mid i\rangle
$$

and $|i\rangle$ is one out of $N$ basis-vectors spanning the singleparticle Hilbert space $\mathcal{H}$.

The frequency integral may be calculated analytically by going into the basis where $\mathcal{G}$ is diagonal, i.e., the eigenbasis of $H_{0}+\Sigma$. We will denote indices in that basis by a tilde, e.g. $\tilde{\mu}$ and the eigenvalues of $H_{0}+\Sigma$ with $\tilde{\epsilon}_{\tilde{\mu}}$. (As $\Sigma$ is Hermitian in the static limit, $\tilde{\epsilon}_{\tilde{\mu}}$ are real.) The basis transform from that 
basis into the basis chosen for observables will be denoted by $V_{i \tilde{\mu}}^{\mathrm{ri}}$. The integral may now be performed analytically, closing the integration loop around the left half-plane,

$$
\begin{aligned}
\rho_{i j} & =\sum_{\tilde{\mu}} V_{i \tilde{\mu}}^{\mathrm{ri}}\left[\frac{1}{2 \pi} \int_{-\infty}^{\infty} d \omega \frac{\mathrm{e}^{\mathrm{i} \omega 0^{+}}}{\mathrm{i} \omega-\tilde{\epsilon}_{\tilde{\mu}}+\mu_{\mathrm{chem}}}\right] V_{\tilde{\mu} j}^{\mathrm{ri},-1} \\
& =\sum_{\tilde{\mu}}^{\mathrm{occ} .} V_{i \tilde{\mu}}^{\mathrm{ri}} V_{\tilde{\mu} j}^{\mathrm{ri},-1}
\end{aligned}
$$

where the summation is now only performed over states below the chemical potential (occupied states).

In order to obtain the result at finite temperature, $T>0$, we must replace the integral by a Matsubara sum, performing the inverse of Eq. (16). The sum may be performed analytically, using the well-known relation

$$
T \sum_{\omega_{n}} \frac{1}{\mathrm{i} \omega_{n}-\xi}=n_{\mathrm{F}}(\xi),
$$

where $n_{\mathrm{F}}$ is the Fermi function. We now obtain

$$
\begin{aligned}
\rho_{i j} & =\sum_{\tilde{\mu}} V_{i \tilde{\mu}}^{\mathrm{ri}}\left[T \sum_{\omega_{n}} \frac{1}{\mathrm{i} \omega_{n}-\tilde{\epsilon}_{\tilde{\mu}}+\mu_{\mathrm{chem}}}\right] V_{\tilde{\mu} j}^{\mathrm{ri},-1} \\
& =\sum_{\tilde{\mu}} V_{i \tilde{\mu}}^{\mathrm{ri}} n_{\mathrm{F}}\left(\tilde{\epsilon}_{\tilde{\mu}}-\mu_{\mathrm{chem}}\right) V_{\tilde{\mu} j}^{\mathrm{ri},-1},
\end{aligned}
$$

which reproduces Eq. (67) for $T \rightarrow 0$.

Another single-particle quantity of interest is the (normalized) density of states (DOS), which may be calculated from the imaginary part of the retarded Green's function after Wick rotation. As we work in the static limit for the self-energy, the Wick rotation is trivial and yields the following expression for the density of states at $T=0$,

$$
\rho(\epsilon)=-\frac{1}{2 \pi N} \Im \sum_{\tilde{\mu}} \frac{1}{\epsilon-\tilde{\epsilon}_{\tilde{\mu}}+\mu_{\mathrm{chem}}+\mathrm{i} 0} .
$$

Finally, in systems with spin rotational invariance the single-particle Green's function is diagonal in spin space and the previously discussed quantities simply acquire a factor of 2 .

\section{Correlator of occupancy numbers $(T=0)$}

Two-particle observables may be rewritten in terms of single- and two-particle Green's functions. In the case of spinless fermions, the correlator of occupancy numbers, $\mathcal{C}_{i j}^{\mathrm{dd}}$, may be rewritten as

$$
\begin{aligned}
\mathcal{C}_{i j}^{\mathrm{dd}} & =\left\langle\hat{\mathrm{n}}_{i} \hat{\mathrm{n}}_{j}\right\rangle=\left\langle\hat{\mathrm{c}}_{i}^{\dagger} \hat{\mathrm{c}}_{i} \hat{\mathrm{c}}_{j}^{\dagger} \hat{\mathrm{c}}_{j}\right\rangle=\left\langle\hat{\mathrm{c}}_{j}^{\dagger} \hat{\mathrm{c}}_{i}^{\dagger} \hat{\mathrm{c}}_{i} \hat{\mathrm{c}}_{j}\right\rangle+\left\langle\hat{\mathrm{c}}_{i}^{\dagger} \hat{\mathrm{c}}_{i}\right\rangle \delta_{i j} \\
& =\mathcal{C}_{i j}^{\mathrm{dd},(2)}+\left\langle\hat{\mathrm{n}}_{i}\right\rangle\left\langle\hat{\mathrm{n}}_{j}\right\rangle-\left\langle\hat{\mathrm{c}}_{i}^{\dagger} \hat{\mathrm{c}}_{j}\right\rangle\left\langle\hat{\mathrm{c}}_{j}^{\dagger} \hat{\mathrm{c}}_{i}\right\rangle+\left\langle\hat{\mathrm{n}}_{i}\right\rangle \delta_{i j},
\end{aligned}
$$

where $\mathcal{C}_{i j}^{\mathrm{dd},(2)}$ is the part of the correlation function arising from the connected two-particle Green's function and thus the vertex. In the case of spinful fermions, the correlator includes a sum over the spin degrees of freedom,

$$
\mathcal{C}_{i j}^{\mathrm{dd}}=\sum_{\sigma \sigma^{\prime}}\left\langle\hat{\mathrm{n}}_{i \sigma} \hat{\mathrm{n}}_{j \sigma^{\prime}}\right\rangle
$$

For systems that obey the full SU(2) symmetry, it reads

$$
\mathcal{C}_{i j}^{\mathrm{dd}}=\mathcal{C}_{i j}^{\mathrm{dd},(2)}+4\left\langle\hat{\mathrm{n}}_{i \sigma}\right\rangle\left\langle\hat{\mathrm{n}}_{j \sigma}\right\rangle-2\left\langle\hat{\mathrm{c}}_{i \sigma}^{\dagger} \hat{\mathrm{c}}_{j \sigma}\right\rangle\left\langle\hat{\mathrm{c}}_{j \sigma}^{\dagger} \hat{\mathrm{c}}_{i \sigma}\right\rangle+2\left\langle\hat{\mathrm{n}}_{i \sigma}\right\rangle \delta_{i j},
$$

where $\sigma$ is an arbitrary spin index that is not summed over, as the single-particle quantities are proportional to $\delta_{\sigma \sigma^{\prime}}$.

We will first derive the expression for $\mathcal{C}_{i j}^{\mathrm{dd},(2)}$ for the spinless case at $T=0$. Since we are looking at static quantities, but our formalism is derived in Matsubara frequency space, we must perform a Fourier transform,

$$
\begin{aligned}
\mathcal{C}_{i j}^{\mathrm{dd},(2)}= & \int \frac{d \omega_{1}}{2 \pi} \int \frac{d \omega_{2}}{2 \pi} \int \frac{d \omega_{3}}{2 \pi} \int \frac{d \omega_{4}}{2 \pi} \\
& \times \mathcal{G}_{i j i j}^{(2, c)}\left(\mathrm{i} \omega_{1}, \mathrm{i} \omega_{2}, \mathrm{i} \omega_{3}, \mathrm{i} \omega_{4}\right),
\end{aligned}
$$

where $\mathcal{G}^{(2, c)}$ is the two-particle connected Green's function. Using the well-known relation between the two-particle connected Green's function and the vertex,

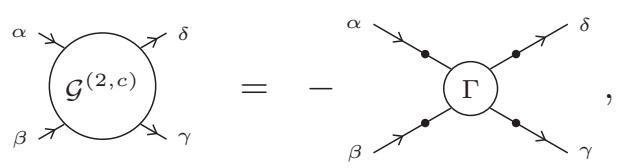

we arrive at

$$
\begin{aligned}
\mathcal{G}_{i j i j}^{(2, c)} & \left(\mathrm{i} \omega_{1}, \mathrm{i} \omega_{2}, \mathrm{i} \omega_{3}, \mathrm{i} \omega_{4}\right) \\
= & -2 \pi \sum_{\alpha \beta \gamma \delta} \sum_{\alpha^{\prime} \beta^{\prime} \gamma^{\prime} \delta^{\prime}} V_{i \alpha^{\prime}}^{\mathrm{rn}} V_{j \beta^{\prime}}^{\mathrm{rn}} \mathcal{G}_{\alpha^{\prime} \alpha}\left(\mathrm{i} \omega_{1}\right) \mathcal{G}_{\beta^{\prime} \beta}\left(\mathrm{i} \omega_{2}\right) \\
& \times \Gamma_{\alpha \beta \gamma \delta} \delta\left(\mathrm{i} \omega_{1}+\mathrm{i} \omega_{2}-\mathrm{i} \omega_{3}-\mathrm{i} \omega_{4}\right) \\
& \times \mathcal{G}_{\gamma \gamma^{\prime}}\left(\mathrm{i} \omega_{3}\right) \mathcal{G}_{\delta \delta^{\prime}}\left(\mathrm{i} \omega_{4}\right) V_{\gamma^{\prime} i}^{\mathrm{rn},-1} V_{\delta^{\prime} j}^{\mathrm{rn},-1} .
\end{aligned}
$$

In order to solve the frequency integral analytically, we again transform into the eigenbasis of $H_{0}+\Sigma$. Equation (76) now reads

$$
\begin{aligned}
& \mathcal{G}_{i j i j}^{(2, c)}\left(\mathrm{i} \omega_{1}, \mathrm{i} \omega_{2}, \mathrm{i} \omega_{3}, \mathrm{i} \omega_{4}\right) \\
&=-2 \pi \sum_{\alpha \beta \gamma \delta} \sum_{\tilde{\mu} \tilde{\nu} \tilde{\rho} \tilde{\sigma}} V_{i \tilde{\mu}}^{\mathrm{ri}} V_{j \tilde{\nu}}^{\mathrm{ri}} \mathcal{G}_{\tilde{\mu} \tilde{\mu}}\left(\mathrm{i} \omega_{1}\right) \mathcal{G}_{\tilde{\nu} \tilde{\nu}}\left(\mathrm{i} \omega_{2}\right) V_{\tilde{\mu} \alpha}^{\mathrm{in}} V_{\tilde{\nu} \beta}^{\mathrm{in}} \\
& \quad \times \Gamma_{\alpha \beta \gamma \delta} \delta\left(\mathrm{i} \omega_{1}+\mathrm{i} \omega_{2}-\mathrm{i} \omega_{3}-\mathrm{i} \omega_{4}\right) \\
& \quad \times V_{\gamma \tilde{\rho}}^{\mathrm{in},-1} V_{\delta \tilde{\sigma}}^{\mathrm{in},-1} \mathcal{G}_{\tilde{\rho} \tilde{\rho}}\left(\mathrm{i} \omega_{3}\right) \mathcal{G}_{\tilde{\sigma} \tilde{\sigma}}\left(\mathrm{i} \omega_{4}\right) V_{\tilde{\rho} i}^{\mathrm{ri},-1} V_{\tilde{\sigma} j}^{\mathrm{ri},-1} .
\end{aligned}
$$

For any given $\tilde{\mu}, \tilde{v}, \tilde{\rho}, \tilde{\sigma}$, we have for the frequency-dependent part

$$
\begin{aligned}
2 \pi \int & \frac{d \omega_{1}}{2 \pi} \int \frac{d \omega_{2}}{2 \pi} \int \frac{d \omega_{3}}{2 \pi} \int \frac{d \omega_{4}}{2 \pi} \mathcal{G}_{\tilde{\mu} \tilde{\mu}}\left(\mathrm{i} \omega_{1}\right) \mathcal{G}_{\tilde{\nu} \tilde{\nu}}\left(\mathrm{i} \omega_{2}\right) \\
& \times \mathcal{G}_{\tilde{\rho} \tilde{\rho}}\left(\mathrm{i} \omega_{3}\right) \mathcal{G}_{\tilde{\sigma} \tilde{\sigma}}\left(\mathrm{i} \omega_{4}\right) \delta\left(\mathrm{i} \omega_{1}+\mathrm{i} \omega_{2}-\mathrm{i} \omega_{3}-\mathrm{i} \omega_{4}\right) \\
= & \int \frac{d \omega_{1}}{2 \pi} \int \frac{d \omega_{2}}{2 \pi} \int \frac{d \omega_{3}}{2 \pi} \mathcal{G}_{\tilde{\mu} \tilde{\mu}}\left(\mathrm{i} \omega_{1}\right) \mathcal{G}_{\tilde{\nu} \tilde{v}}\left(\mathrm{i} \omega_{2}\right) \\
& \times \mathcal{G}_{\tilde{\rho} \tilde{\rho}}\left(\mathrm{i} \omega_{3}\right) \mathcal{G}_{\tilde{\sigma} \tilde{\sigma}}\left(\mathrm{i}\left(\omega_{1}+\omega_{2}-\omega_{3}\right)\right) .
\end{aligned}
$$

Using the convention that $\tilde{\epsilon}_{\tilde{\mu}}$ is the $\tilde{\mu}$ th eigenvalue of $H_{0}+\Sigma$, we may now write

$$
\mathcal{G}_{\tilde{\mu} \tilde{\mu}}\left(\mathrm{i} \omega_{1}\right)=\frac{1}{\mathrm{i} \omega_{1}-\tilde{\epsilon}_{\tilde{\mu}}+\mu_{\mathrm{chem}}}=: \frac{1}{\mathrm{i} \omega_{1}-\tilde{\xi}_{\tilde{\mu}}} .
$$


All occurring integrals are of similar form and may be solved by simply closing the integration loop around the left complex half-plane,

$$
\int \frac{d \omega}{2 \pi} \frac{1}{\mathrm{i} \omega-z} \frac{1}{\mathrm{i} \omega-\xi}=\frac{g(z, \xi)}{z-\xi} .
$$

The exact result of the integral will depend on the position of each of the poles $\{z, \xi\}$ relative to the integration loop. If they are either both inside or both outside, the integral gives zero (either the residues cancel or there are no poles inside the loop), there is only a contribution if there is just a single pole inside the loop. The residue is always $\pm(z-\xi)^{-1}$. Therefore we define $g(z, \xi)$ to keep track of the correct sign. It may be represented as

$$
\begin{aligned}
g(z, \xi) & =-g(\xi, z) \\
& =\Theta_{\Re}(-z) \Theta_{\Re}(\xi)-\Theta_{\Re}(z) \Theta_{\Re}(-\xi),
\end{aligned}
$$

where $\Theta_{\Re}(z)$ is the Heaviside step function of the real part of $z$.

Performing the first integral over $\omega_{1}$, we have

$$
\begin{aligned}
& \int \frac{d \omega_{1}}{2 \pi} \frac{1}{\mathrm{i} \omega_{1}-\tilde{\xi}_{\tilde{\mu}}} \frac{1}{\mathrm{i} \omega_{1}-\left(\tilde{\xi}_{\tilde{\sigma}}-\mathrm{i} \omega_{2}+\mathrm{i} \omega_{3}\right)} \\
& =\frac{g\left(\tilde{\xi}_{\tilde{\mu}}, \tilde{\xi}_{\tilde{\sigma}}+\mathrm{i}\left(\omega_{3}-\omega_{2}\right)\right)}{\tilde{\xi}_{\tilde{\mu}}-\tilde{\xi}_{\tilde{\sigma}}+\mathrm{i} \omega_{2}-\mathrm{i} \omega_{3}}
\end{aligned}
$$

The expression $g\left(\tilde{\xi}_{\tilde{\mu}}, \tilde{\xi}_{\tilde{\sigma}}+\mathrm{i}\left(\omega_{3}-\omega_{2}\right)\right)$ may be simplified further, since for real $\omega_{2,3}$, it is equal to $g\left(\tilde{\xi}_{\tilde{\mu}}, \tilde{\xi}_{\tilde{\sigma}}\right) .^{4}$ Applying this result sequentially, the integral in Eq. (78) has the result

$$
\frac{g\left(\tilde{\xi}_{\tilde{\mu}}, \tilde{\xi}_{\tilde{\sigma}}\right) g\left(\tilde{\xi}_{\tilde{\sigma}}-\tilde{\xi}_{\tilde{\mu}}, \tilde{\xi}_{\tilde{v}}\right) g\left(\tilde{\xi}_{\tilde{\mu}}+\tilde{\xi}_{\tilde{v}}-\tilde{\xi}_{\tilde{\sigma}}, \tilde{\xi}_{\tilde{\rho}}\right)}{\tilde{\xi}_{\tilde{\mu}}+\tilde{\xi}_{\tilde{v}}-\tilde{\xi}_{\tilde{\rho}}-\tilde{\xi}_{\tilde{\sigma}}} .
$$

Further simplification is possible: if $\Re \tilde{\xi}_{\tilde{\mu}}>0$, then $\Re \tilde{\xi}_{\tilde{\sigma}}$ must be less than zero, or the contribution vanishes. In that case, it follows that $\Re\left(\tilde{\xi}_{\tilde{\sigma}}-\tilde{\xi}_{\tilde{\mu}}\right)<0$, and we may deduce in the same way that $\mathfrak{R} \tilde{\xi}_{\tilde{v}}$ should be greater than zero. Finally, $\Re\left(\tilde{\xi}_{\tilde{\mu}}+\tilde{\xi}_{\tilde{v}}-\tilde{\xi}_{\tilde{\sigma}}\right)>0$ leads to the conclusion that $\Re \tilde{\xi}_{\tilde{\rho}}<0$. On the other hand, if $\Re \tilde{\xi}_{\tilde{\mu}}<0$, the analogous argument can be made with flipped inequalities. The only nonzero contributions arise from combinations where the real parts of $\tilde{\xi}_{\tilde{\mu}}$ and $\tilde{\xi}_{\tilde{v}}$ have the same sign, but have the opposite sign to both $\tilde{\xi}_{\tilde{\rho}}$ and $\tilde{\xi}_{\tilde{\sigma}}$. Using this result, Eq. (74) now reads

$$
\begin{aligned}
\mathcal{C}_{i j}^{\mathrm{dd},(2)}= & \sum_{\alpha \beta \gamma \delta}\left[\sum_{\substack{\tilde{\mu}, \tilde{\nu} \in \mathcal{H}_{e} \\
\tilde{\rho}, \tilde{\sigma} \in \mathcal{H}_{h}}}-\sum_{\substack{\tilde{\mu}, \tilde{\nu} \in \mathcal{H}_{h} \\
\tilde{\rho}, \tilde{\sigma} \in \mathcal{H}_{e}}}\right] \\
& \times V_{i \tilde{\mu}}^{\mathrm{ri}} V_{j \tilde{\nu}}^{\mathrm{ri}} \frac{1}{\tilde{\epsilon}_{\tilde{\mu}}+\tilde{\epsilon}_{\tilde{\nu}}-\tilde{\epsilon}_{\tilde{\rho}}-\tilde{\epsilon}_{\tilde{\sigma}}} V_{\tilde{\rho} i}^{\mathrm{ri},-1} V_{\tilde{\sigma} j}^{\mathrm{ri},-1} \\
& \times V_{\tilde{\mu} \alpha}^{\mathrm{in}} V_{\tilde{\nu} \beta}^{\mathrm{in}} \Gamma_{\alpha \beta \gamma \delta} V_{\gamma \tilde{\rho}}^{\mathrm{in},-1} V_{\delta \tilde{\sigma}}^{\mathrm{in},-1},
\end{aligned}
$$

where $\mathcal{H}_{e}$ is the subspace where $\tilde{\epsilon}-\mu_{\text {chem }}<0$ ("electrons") and $\mathcal{H}_{h}$ the subspace where $\tilde{\epsilon}+\mu_{\text {chem }}>0$ ("holes").

\footnotetext{
${ }^{4}$ Note that while closing the integrals over $\omega_{2,3}$, those frequencies may obtain an imaginary part, but since semicircle contour parts have a vanishing contribution to the integral itself, this may be ignored.
}

\section{Correlator of occupancy numbers $(T>0)$}

At finite temperatures $T>0$, the result is very similar. To derive it, we need to replace the integrals in Eq. (78) by Matsubara sums according to the inverse of Eqs. (16) and (17),

$$
\begin{aligned}
T & \sum_{\omega_{n}} T \sum_{\omega_{m}} T \sum_{\omega_{n^{\prime}}} \mathcal{G}_{\tilde{\mu} \tilde{\mu}}\left(\mathrm{i} \omega_{n}\right) \mathcal{G}_{\tilde{\nu} \tilde{\nu}}\left(\mathrm{i} \omega_{m}\right) \\
& \times \mathcal{G}_{\tilde{\rho} \tilde{\rho}}\left(\mathrm{i} \omega_{n^{\prime}}\right) \mathcal{G}_{\tilde{\sigma} \tilde{\sigma}}\left(\mathrm{i}\left(\omega_{n}+\omega_{m}-\omega_{n^{\prime}}\right)\right) .
\end{aligned}
$$

Inserting Eq. (79) into this expression, we may now perform the Matsubara sums analytically, which are of the form

$$
T \sum_{\omega_{n}} \frac{1}{\mathrm{i} \omega_{n}-z} \frac{1}{\mathrm{i} \omega_{n}-\xi}=\frac{n_{\mathrm{F}}(z)-n_{\mathrm{F}}(\xi)}{z-\xi},
$$

where $n_{\mathrm{F}}$ is the Fermi function. We note that due to its periodicity we have $n_{\mathrm{F}}\left(\tilde{\xi} \pm \mathrm{i} \omega_{n^{\prime}}\right)=n_{\mathrm{F}}(\tilde{\xi})$ if $\omega_{n^{\prime}}$ is a Matsubara frequency, so we may simplify the numerator again. Equation (85) is thus equal to

$$
\begin{aligned}
& {\left[n_{\mathrm{F}}\left(\tilde{\xi}_{\tilde{\mu}}\right)-n_{\mathrm{F}}\left(\tilde{\xi}_{\tilde{\sigma}}\right)\right]\left[n_{\mathrm{F}}\left(\tilde{\xi}_{\tilde{\sigma}}-\tilde{\xi}_{\tilde{\mu}}\right)-n_{\mathrm{F}}\left(\tilde{\xi}_{\tilde{v}}\right)\right]} \\
& \times \frac{\left[n_{\mathrm{F}}\left(\tilde{\xi}_{\tilde{\mu}}+\tilde{\xi}_{\tilde{v}}-\tilde{\xi}_{\tilde{\sigma}}\right)-n_{\mathrm{F}}\left(\tilde{\xi}_{\tilde{\rho}}\right)\right]}{\tilde{\xi}_{\tilde{\mu}}+\tilde{\xi}_{\tilde{v}}-\tilde{\xi}_{\tilde{\rho}}-\tilde{\xi}_{\tilde{\sigma}}} .
\end{aligned}
$$

Therefore, we have

$$
\begin{aligned}
\mathcal{C}_{i j}^{\mathrm{dd},(2)}= & \sum_{\alpha \beta \gamma \delta} \sum_{\tilde{\mu} \tilde{\nu} \tilde{\rho} \tilde{\sigma}} V_{i \tilde{\mu}}^{\mathrm{ri}} V_{j \tilde{v}}^{\mathrm{ri}} \frac{1}{\tilde{\epsilon}_{\tilde{\mu}}+\tilde{\epsilon}_{\tilde{\nu}}-\tilde{\epsilon}_{\tilde{\rho}}-\tilde{\epsilon}_{\tilde{\sigma}}} \\
& \times\left[n_{\mathrm{F}}\left(\tilde{\xi}_{\tilde{\sigma}}\right)-n_{\mathrm{F}}\left(\tilde{\xi}_{\tilde{\mu}}\right)\right]\left[n_{\mathrm{F}}\left(\tilde{\xi}_{\tilde{\sigma}}-\tilde{\xi}_{\tilde{\mu}}\right)-n_{\mathrm{F}}\left(\tilde{\xi}_{\tilde{v}}\right)\right] \\
& \times\left[n_{\mathrm{F}}\left(\tilde{\xi}_{\tilde{\mu}}+\tilde{\xi}_{\tilde{\nu}}-\tilde{\xi}_{\tilde{\sigma}}\right)-n_{\mathrm{F}}\left(\tilde{\xi}_{\tilde{\rho}}\right)\right] V_{\tilde{\rho} i}^{\mathrm{ri},-1} V_{\tilde{\sigma} j}^{\mathrm{ri},-1} \\
& \times V_{\tilde{\mu} \alpha}^{\mathrm{in}} V_{\tilde{\nu} \beta}^{\mathrm{in}} \Gamma_{\alpha \beta \gamma \delta} V_{\gamma \tilde{\rho}}^{\mathrm{in},-1} V_{\delta \tilde{\sigma}}^{\mathrm{in},-1} .
\end{aligned}
$$

For orbitals far away from the Fermi energy, $|\tilde{\xi}| \gg T$, this expression goes over into the expression for $T=0$ and we arrive at Eq. (84) again.

\section{Correlator of occupancy numbers (systems with spin)}

In systems with spin we must also sum over two spin indices when calculating $\mathcal{C}_{i j}^{\mathrm{dd},(2)}$. We replace all orbital indices in Eq. (88) by pairs of orbital and spin indices, $\alpha \rightarrow(\alpha, \sigma)$. For systems with $\mathrm{SU}(2)$ symmetry all single-particle quantities are diagonal in spin space, so after performing sums over all the relevant Kronecker $\delta$ s, we have

$$
\begin{aligned}
\mathcal{C}_{i j}^{\mathrm{dd},(2)}= & \sum_{\sigma \sigma^{\prime}} \sum_{\alpha \beta \gamma \delta} \sum_{\tilde{\mu} \tilde{\nu} \tilde{\rho} \tilde{\sigma}} V_{i \tilde{\mu}}^{\mathrm{ri}} V_{j \tilde{\nu}}^{\mathrm{ri}} \frac{1}{\tilde{\epsilon}_{\tilde{\mu}}+\tilde{\epsilon}_{\tilde{\nu}}-\tilde{\epsilon}_{\tilde{\rho}}-\tilde{\epsilon}_{\tilde{\sigma}}} \\
& \times\left[n_{\mathrm{F}}\left(\tilde{\xi}_{\tilde{\sigma}}\right)-n_{\mathrm{F}}\left(\tilde{\xi}_{\tilde{\mu}}\right)\right]\left[n_{\mathrm{F}}\left(\tilde{\xi}_{\tilde{\sigma}}-\tilde{\xi}_{\tilde{\mu}}\right)-n_{\mathrm{F}}\left(\tilde{\xi}_{\tilde{v}}\right)\right] \\
& \times\left[n_{\mathrm{F}}\left(\tilde{\xi}_{\tilde{\mu}}+\tilde{\xi}_{\tilde{v}}-\tilde{\xi}_{\tilde{\sigma}}\right)-n_{\mathrm{F}}\left(\tilde{\xi}_{\tilde{\rho}}\right)\right] V_{\tilde{\rho} i}^{\mathrm{ri},-1} V_{\tilde{\sigma} j}^{\mathrm{ri},-1} \\
& \times V_{\tilde{\mu} \alpha}^{\mathrm{in}} V_{\tilde{\nu} \beta}^{\mathrm{in}} \Gamma_{(\alpha, \sigma)\left(\beta, \sigma^{\prime}\right)(\gamma, \sigma)\left(\delta, \sigma^{\prime}\right)} V_{\gamma \tilde{\rho}}^{\mathrm{in},-1} V_{\delta \tilde{\sigma}}^{\mathrm{in},-1} .
\end{aligned}
$$

Inserting Eq. (44), we may perform the summation over the remaining spin indices and arrive at

$$
\begin{aligned}
\mathcal{C}_{i j}^{\mathrm{dd},(2)}= & \sum_{\alpha \beta \gamma \delta} \sum_{\tilde{\mu} \tilde{\nu} \tilde{\rho} \tilde{\sigma}} V_{i \tilde{\mu}}^{\mathrm{ri}} V_{j \tilde{v}}^{\mathrm{ri}} \frac{1}{\tilde{\epsilon}_{\tilde{\mu}}+\tilde{\epsilon}_{\tilde{\nu}}-\tilde{\epsilon}_{\tilde{\rho}}-\tilde{\epsilon}_{\tilde{\sigma}}} \\
& \times\left[n_{\mathrm{F}}\left(\tilde{\xi}_{\tilde{\sigma}}\right)-n_{\mathrm{F}}\left(\tilde{\xi}_{\tilde{\mu}}\right)\right]\left[n_{\mathrm{F}}\left(\tilde{\xi}_{\tilde{\sigma}}-\tilde{\xi}_{\tilde{\mu}}\right)-n_{\mathrm{F}}\left(\tilde{\xi}_{\tilde{v}}\right)\right]
\end{aligned}
$$




$$
\begin{aligned}
& \times\left[n_{\mathrm{F}}\left(\tilde{\xi}_{\tilde{\mu}}+\tilde{\xi}_{\tilde{\nu}}-\tilde{\xi}_{\tilde{\sigma}}\right)-n_{\mathrm{F}}\left(\tilde{\xi}_{\tilde{\rho}}\right)\right] V_{\tilde{\rho} i}^{\mathrm{ri},-1} V_{\tilde{\sigma} j}^{\mathrm{ri},-1} \\
& \times V_{\tilde{\mu} \alpha}^{\mathrm{in}} V_{\tilde{\nu} \beta}^{\mathrm{in}}\left[2 \Gamma_{\alpha \beta \gamma \delta}^{\mathrm{s}}-4 \Gamma_{\alpha \beta \delta \gamma}^{\mathrm{s}}\right] V_{\gamma \tilde{\rho}}^{\mathrm{in},-1} V_{\delta \tilde{\sigma}}^{\mathrm{in},-1} .
\end{aligned}
$$

At $T=0$, the result is analogously given by

$$
\begin{aligned}
\mathcal{C}_{i j}^{\mathrm{dd},(2)}= & \sum_{\alpha \beta \gamma \delta}\left[\sum_{\substack{\tilde{\mu}, \tilde{\nu} \in \mathcal{H}_{e} \\
\tilde{\rho}, \tilde{\sigma} \in \mathcal{H}_{h}}}-\sum_{\substack{\tilde{\mu}, \tilde{\nu} \in \mathcal{H}_{h} \\
\tilde{\rho}, \tilde{\sigma} \in \mathcal{H}_{e}}}\right] \\
& \times V_{i \tilde{\mu}}^{\mathrm{ri}} V_{j \tilde{\nu}}^{\mathrm{ri}} \frac{1}{\tilde{\epsilon}_{\tilde{\mu}}+\tilde{\epsilon}_{\tilde{\nu}}-\tilde{\epsilon}_{\tilde{\rho}}-\tilde{\epsilon}_{\tilde{\sigma}}} V_{\tilde{\rho} i}^{\mathrm{ri},-1} V_{\tilde{\sigma} j}^{\mathrm{ri},-1} \\
& \times V_{\tilde{\mu} \alpha}^{\mathrm{in}} V_{\tilde{\nu} \beta}^{\mathrm{in}}\left[2 \Gamma_{\alpha \beta \gamma \delta}^{\mathrm{s}}-4 \Gamma_{\alpha \beta \delta \gamma}^{\mathrm{s}}\right] V_{\gamma \tilde{\rho}}^{\mathrm{in},-1} V_{\delta \tilde{\sigma}}^{\mathrm{in},-1} .
\end{aligned}
$$

\section{Spin-spin correlator}

In contrast to the expectation value of $\mathbf{S}_{i}$, the expectation value of $\mathbf{S}_{i} \cdot \mathbf{S}_{j}$ does not automatically vanish in systems with $\mathrm{SU}(2)$ symmetry. Using

$$
\hat{\mathbf{S}}_{i}=\sum_{\sigma \sigma^{\prime}} \hat{\mathrm{c}}_{i \sigma}^{\dagger} \vec{\tau}_{\sigma \sigma^{\prime}} \hat{\mathbf{c}}_{i \sigma^{\prime}},
$$

where $\vec{\tau}$ are the Pauli matrices and the identity

$$
\sum_{k=0}^{3} \tau_{\sigma \sigma^{\prime}}^{k} \tau_{\bar{\sigma} \bar{\sigma}^{\prime}}^{k}=2 \delta_{\sigma \bar{\sigma}^{\prime}} \delta_{\sigma^{\prime} \bar{\sigma}},
$$

we may write

$$
\begin{aligned}
\mathcal{C}_{i j}^{\mathrm{ss}}:= & \left\langle\mathbf{S}_{i} \cdot \mathbf{S}_{j}\right\rangle \\
= & \sum_{k} \sum_{\sigma \sigma^{\prime}} \sum_{\bar{\sigma} \bar{\sigma}^{\prime}} \tau_{\sigma \sigma^{\prime}}^{k} \tau_{\bar{\sigma} \bar{\sigma}^{\prime}}^{k}\left\langle\hat{\mathrm{c}}_{i \sigma}^{\dagger} \hat{\mathrm{c}}_{i \sigma^{\prime}} \hat{\mathrm{c}}_{j \bar{\sigma}}^{\dagger} \hat{\mathrm{c}}_{j \bar{\sigma}^{\prime}}\right\rangle \\
= & 2 \sum_{\sigma \sigma^{\prime}}\left\langle\hat{\mathrm{c}}_{i \sigma}^{\dagger} \hat{\mathrm{c}}_{i \sigma^{\prime}} \hat{\mathrm{c}}_{j \sigma^{\prime}}^{\dagger} \hat{\mathrm{c}}_{j \sigma}\right\rangle-\left\langle\hat{\mathrm{n}}_{i} \hat{\mathrm{n}}_{j}\right\rangle \\
= & 2 \sum_{\sigma \sigma^{\prime}}\left\langle\hat{\mathrm{c}}_{i \sigma}^{\dagger} \hat{\mathrm{c}}_{j \sigma^{\prime}}^{\dagger} \hat{\mathrm{c}}_{j \sigma} \hat{\mathrm{c}}_{i \sigma^{\prime}}\right\rangle-\left\langle\hat{\mathrm{n}}_{i} \hat{\mathrm{n}}_{j}\right\rangle-4 \delta_{i j}\left\langle\hat{\mathrm{n}}_{i}\right\rangle \\
= & \mathcal{C}_{i j}^{\mathrm{ss},(2)}-\left\langle\hat{\mathrm{n}}_{i} \hat{\mathrm{n}}_{j}\right\rangle-4 \delta_{i j}\left\langle\hat{\mathrm{n}}_{i}\right\rangle \\
& +2 \sum_{\sigma \sigma^{\prime}}\left[\left\langle\hat{\mathrm{c}}_{j \sigma^{\prime}}^{\dagger} \hat{\mathrm{c}}_{j \sigma}\right\rangle\left\langle\hat{\mathrm{c}}_{i \sigma}^{\dagger} \hat{\mathrm{c}}_{i \sigma^{\prime}}\right\rangle-\left\langle\hat{\mathrm{c}}_{i \sigma}^{\dagger} \hat{\mathrm{c}}_{j \sigma}\right\rangle\left\langle\hat{\mathrm{c}}_{j \sigma^{\prime}}^{\dagger} \hat{\mathrm{c}}_{i \sigma^{\prime}}\right\rangle\right] \\
= & \mathcal{C}_{i j}^{\mathrm{ss},(2)}+4\left\langle\hat{\mathrm{n}}_{i \sigma}\right\rangle\left\langle\hat{\mathrm{n}}_{j \sigma}\right\rangle-8\left\langle\hat{\mathrm{c}}_{i \sigma}^{\dagger} \hat{\mathrm{c}}_{j \sigma}\right\rangle\left\langle\hat{\mathrm{c}}_{j \sigma}^{\dagger} \hat{\mathrm{c}}_{i \sigma}\right\rangle \\
& -\left\langle\hat{\mathrm{n}}_{i} \hat{\mathrm{n}}_{j}\right\rangle-4 \delta_{i j}\left\langle\hat{\mathrm{n}}_{i}\right\rangle .
\end{aligned}
$$

Inserting Eq. (73), several terms cancel and we arrive at

$$
\mathcal{C}_{i j}^{\mathrm{ss}}=\mathcal{C}_{i j}^{\mathrm{ss},(2)}-\mathcal{C}_{i j}^{\mathrm{dd},(2)}-6\left(\left\langle\hat{\mathrm{c}}_{i \sigma}^{\dagger} \hat{\mathrm{c}}_{j \sigma}\right\rangle\left\langle\hat{\mathrm{c}}_{j \sigma}^{\dagger} \hat{\mathrm{c}}_{i \sigma}\right\rangle+\delta_{i j}\left\langle\hat{\mathrm{n}}_{i}\right\rangle\right) .
$$

The expression for $\mathcal{C}_{i j}^{\text {ss, (2) }}$ may be derived in the same manner as the expression for $\mathcal{C}_{i j}^{\mathrm{dd},(2)}$. At finite temperatures, it reads

$$
\begin{aligned}
\mathcal{C}_{i j}^{\mathrm{ss},(2)}= & 2 \sum_{\alpha \beta \gamma \delta} \sum_{\tilde{\mu} \tilde{\nu} \tilde{\rho} \tilde{\sigma}} V_{i \tilde{\mu}}^{\mathrm{ri}} V_{j \tilde{v}}^{\mathrm{ri}} \frac{1}{\tilde{\epsilon}_{\tilde{\mu}}+\tilde{\epsilon}_{\tilde{v}}-\tilde{\epsilon}_{\tilde{\rho}}-\tilde{\epsilon}_{\tilde{\sigma}}} \\
& \times\left[n_{\mathrm{F}}\left(\tilde{\xi}_{\tilde{\sigma}}\right)-n_{\mathrm{F}}\left(\tilde{\xi}_{\tilde{\mu}}\right)\right]\left[n_{\mathrm{F}}\left(\tilde{\xi}_{\tilde{\sigma}}-\tilde{\xi}_{\tilde{\mu}}\right)-n_{\mathrm{F}}\left(\tilde{\xi}_{\tilde{v}}\right)\right] \\
& \times\left[n_{\mathrm{F}}\left(\tilde{\xi}_{\tilde{\mu}}+\tilde{\xi}_{\tilde{v}}-\tilde{\xi}_{\tilde{\sigma}}\right)-n_{\mathrm{F}}\left(\tilde{\xi}_{\tilde{\rho}}\right)\right] V_{\tilde{\rho} i}^{\mathrm{ri},-1} V_{\tilde{\sigma} j}^{\mathrm{ri},-1} \\
& \times V_{\tilde{\mu} \alpha}^{\mathrm{in}} V_{\tilde{\nu} \beta}^{\mathrm{in}}\left[4 \Gamma_{\alpha \beta \gamma \delta}^{\mathrm{s}}-2 \Gamma_{\alpha \beta \delta \gamma}^{\mathrm{s}}\right] V_{\gamma \tilde{\rho}}^{\mathrm{in},-1} V_{\delta \tilde{\sigma}}^{\mathrm{in},-1} .
\end{aligned}
$$

As one is often interested in both the occupation number and spin correlators, we note that the expression for the difference between $\mathcal{C}_{i j}^{\text {ss,(2) }}$ and $\mathcal{C}_{i j}^{\mathrm{dd},(2)}$ simplifies slightly,

$$
\begin{aligned}
\mathcal{C}_{i j}^{\text {ss, },(2)}= & \mathcal{C}_{i j}^{\mathrm{ss},(2)}-\mathcal{C}_{i j}^{\mathrm{dd},(2)} \\
= & 6 \sum_{\alpha \beta \gamma \delta} \sum_{\tilde{\mu} \tilde{\nu} \tilde{\rho} \tilde{\sigma}} V_{i \tilde{\mu}}^{\mathrm{ri}} V_{j \tilde{v}}^{\mathrm{ri}} \frac{1}{\tilde{\epsilon}_{\tilde{\mu}}+\tilde{\epsilon}_{\tilde{\nu}}-\tilde{\epsilon}_{\tilde{\rho}}-\tilde{\epsilon}_{\tilde{\sigma}}} \\
& \times\left[n_{\mathrm{F}}\left(\tilde{\xi}_{\tilde{\sigma}}\right)-n_{\mathrm{F}}\left(\tilde{\xi}_{\tilde{\mu}}\right)\right]\left[n_{\mathrm{F}}\left(\tilde{\xi}_{\tilde{\sigma}}-\tilde{\xi}_{\tilde{\mu}}\right)-n_{\mathrm{F}}\left(\tilde{\xi}_{\tilde{\nu}}\right)\right] \\
& \times\left[n_{\mathrm{F}}\left(\tilde{\xi}_{\tilde{\mu}}+\tilde{\xi}_{\tilde{\nu}}-\tilde{\xi}_{\tilde{\sigma}}\right)-n_{\mathrm{F}}\left(\tilde{\xi}_{\tilde{\rho}}\right)\right] V_{\tilde{\rho} i}^{\mathrm{ri},-1} V_{\tilde{\sigma} j}^{\mathrm{ri},-1} \\
& \times V_{\tilde{\mu} \alpha}^{\mathrm{in}} V_{\tilde{\nu} \beta}^{\mathrm{in}} \Gamma_{\alpha \beta \gamma \delta}^{\mathrm{s}} V_{\gamma \tilde{\rho}}^{\mathrm{in},-1} V_{\delta \tilde{\sigma}}^{\mathrm{in},-1} .
\end{aligned}
$$

At $T=0$, the expression reads

$$
\begin{aligned}
\mathcal{C}_{i j}^{\prime s s,(2)}= & 6 \sum_{\alpha \beta \gamma \delta}\left[\sum_{\substack{\tilde{\mu}, \tilde{\nu} \in \mathcal{H}_{e} \\
\tilde{\rho}, \tilde{\sigma} \in \mathcal{H}_{h}}}-\sum_{\substack{\tilde{\mu}, \tilde{\nu} \in \mathcal{H}_{h} \\
\tilde{\rho}, \tilde{\sigma} \in \mathcal{H}_{e}}}\right] \\
& \times V_{i \tilde{\mu}}^{\mathrm{ri}} V_{j \tilde{\nu}}^{\mathrm{ri}} \frac{1}{\tilde{\epsilon}_{\tilde{\mu}}+\tilde{\epsilon}_{\tilde{\nu}}-\tilde{\epsilon}_{\tilde{\rho}}-\tilde{\epsilon}_{\tilde{\sigma}}} V_{\tilde{\rho} i}^{\mathrm{ri},-1} V_{\tilde{\sigma} j}^{\mathrm{ri},-1} \\
& \times V_{\tilde{\mu} \alpha}^{\mathrm{in}} V_{\tilde{\nu} \beta}^{\mathrm{in}} \Gamma_{\alpha \beta \gamma \delta}^{\mathrm{s}} V_{\gamma \tilde{\rho}}^{\mathrm{in},-1} V_{\delta \tilde{\sigma}}^{\mathrm{in},-1} .
\end{aligned}
$$

\section{F. Reducting the Hilbert space size: active-space approximation (ASA)}

The flow equations for the self-energy and the vertex, even in their simplest form Eqs. (31) and (32), are still computationally challenging. In translationally invariant systems, simplifications arise, because the vertex only depends on three momenta, the fourth given by momentum conservation. Moreover, one only tracks momenta near the Fermi surface. The Brillouin zone is divided into patches each containing a single tracked momentum and the interaction vertex $\Gamma$ is only calculated at these momenta. Whenever it needs to be evaluated for other momenta, the other momentum is replaced by the tracked one located within the same patch (coarse graining) [13]. In the absence of periodicity, this kind of patching is not possible, since there is no well-defined concept of a Fermi surface.

For systems without translational symmetries, we here propose an approach alternative to Fermi-surface patching for reducing the number of explicit degrees of freedom. Similar to the patching scheme, we define an "active space" $\mathcal{H}_{M}$ of (effective) single-particle states near the chemical potential that are kept. In our case $\mathcal{H}_{M}$ simply contains the $M$ orbitals closest to the chemical potential, $\mu_{\text {chem }}$, (e.g. half above and half below); see Fig. 2 for details. We will refer to this approach in a loose manner of speaking as "active-space approximation" (ASA).

Within ASA external indices of the flow equation for the vertex, Eq. (32), only refer to a reduced number of states. In summations over the full single-particle Hilbert space, we adopt the approximation scheme

$$
\Gamma_{\alpha \beta \gamma \delta}^{\Lambda} \rightarrow \begin{cases}\Gamma_{\alpha \beta \gamma \delta}^{\Lambda} & \{\alpha, \beta, \gamma, \delta\} \subseteq \mathcal{H}_{M} \\ U_{\alpha \beta \gamma \delta} & \text { otherwise }\end{cases}
$$




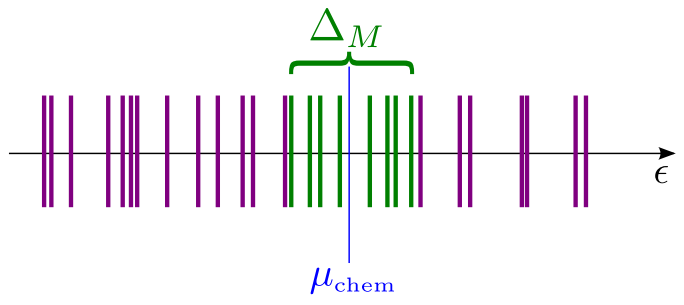

FIG. 2. Active space: selection of $M$ orbitals (green) around the chemical potential, $\mu_{\text {chem }}$, for which the vertex will be renormalized in the active space approximation (ASA). The self-energy will still be renormalized for all $N$ orbitals, including the remaining (purple) ones.

To simplify the notation, in the following, we label states from the active space $\mathcal{H}_{M}$ with barred indices, e.g., $\bar{\alpha}$, whereas states from the full set of orbitals are denoted without bars, e.g., $\alpha$.

We comment on the choice for $M$ at a given system size. As long as mostly the states close to the Fermi energy are important for screening (as is also assumed in applications of the FRG for translationally invariant systems), we can argue that the number of states necessarily kept in $\mathcal{H}_{M}, M$, should grow sublinearly with the total number of orbitals, $N$. We remind ourselves that in a translationally invariant system, the Fermi surface has dimensionality $(d-1)$ within the $d$-dimensional Brillouin zone. Since the number of states in the Brillouin zone grows as $L^{d}$, but the number of states on a surface within that space grows as $L^{d-1}$, we suggest the number of states required should be proportional to $L^{d-1}$, which can be rewritten as $L^{d-1}=\left(L^{d}\right)^{(d-1) / d}=N^{1-1 / d}$. To the extent that $M$ scales, the same also for generic systems, we have $M \sim N^{1-1 / d}$, implying $M \sim N^{1 / 2}$ in 2D. In Sec. IV, we will establish the efficacy of the ASA and also revisit the system size scaling.

\section{G. Runaway flow}

At present, one of the main applications of $k \mathrm{FRG}$ is the study of phase diagrams, because an unbiased view of competing instabilities of the system is provided. In parameter regimes where the system shows a phase transition, the instabilities pertaining to the new phase lead to "runaway flow": at a critical scale, $\Lambda_{c}$, the integration of the RG equations exhibits matrix elements of the interaction vertex that diverge. The physical nature of the instability reveals itself in what matrix element actually shows the strongest divergence. This property of the FRG has been used very successfully to study the phase diagram of a multitude of systems, for an overview see Ref. [5]. With $\epsilon$ FRG one needs to keep in mind that the eigenstate representation is not based on plane waves. Therefore the physics of individual vertex elements may not be as transparent as it is in the clean case. Hence it can be helpful to calculate two-particle correlators at $\Lambda_{c}$ to support interpretations of the precise nature of the instability.

We mention that cases exist in which competing order parameters influence each other (such as antiferromagnetism and $d$-wave superconductivity). Strategies how to deal with this situation have been developed within $k$ FRG. Ideally, one should continue the flow to $\Lambda \rightarrow 0$ to obtain information about the true phase diagram of the system. This may be done in principle, e.g., by introducing an infinitesimal symmetry-breaking term that grows under the RG flow, as has been done for superconductivity [74]. Alternatively, one may calculate the flow for the combined Bose-Fermi system, where fermions were decoupled via a Hubbard-Stratonovich transformation [75].

\section{IMPLEMENTATION}

We implement the $\mathrm{FRG}$ procedure in $\mathrm{C}++$, using the Eigen linear algebra library [76] for matrix products and the HDF5 file format [77] for storage. We employ the OPENMP 3.1 standard [78] for parallelization.

\section{A. Computational details}

The computational complexity of the self-energy flow, Eqs. (31), (46), and (62), is given by $\mathcal{O}\left(N^{4}\right)$ - two loops for each of the outer indices, two loops for the contraction with the nondiagonal single-scale propagator. At first glance, the flow of the vertex, e.g., Eq. (32) appears to have a complexity of $\mathcal{O}\left(N^{8}\right)$. However, one may define intermediate products, $I^{\mathrm{c}, \pm}$ and $I^{\mathrm{ph}, \pm}$

$$
\begin{aligned}
& I_{\mu \rho \bar{\gamma} \bar{\delta}}^{\mathrm{c},+}=\sum_{\nu} P_{\mu \nu}^{\Lambda, s}(\Lambda) \Gamma_{\nu \rho \bar{\gamma} \bar{\delta}}^{\Lambda}, \\
& I_{\bar{\alpha} \bar{\beta} \rho \mu}^{\mathrm{c},-}=\sum_{\sigma} P_{\rho \sigma}^{\Lambda, s}(-\Lambda) \Gamma_{\bar{\alpha} \bar{\beta} \sigma \mu}^{\Lambda}, \\
& I_{\bar{\alpha} \nu \bar{\gamma} \sigma}^{\mathrm{ph}, \pm}=\sum_{\rho} \Gamma_{\bar{\alpha} \nu \bar{\gamma} \rho}^{\Lambda} P_{\rho \sigma}^{\Lambda, s}( \pm \Lambda),
\end{aligned}
$$

where each of these partial diagrams has a complexity of $\mathcal{O}\left(N^{5}\right)$. The flow equation for the vertex now reads

$$
\begin{aligned}
\frac{d}{d \Lambda} \Gamma_{\bar{\alpha} \bar{\beta} \bar{\gamma} \bar{\delta}}^{\Lambda}= & -\frac{1}{2 \pi} \sum_{\mu \rho}\left\{I_{\mu \rho \bar{\gamma} \bar{\delta}}^{\mathrm{c},+} I_{\bar{\alpha} \bar{\beta} \rho \mu}^{\mathrm{c},-}+I_{\bar{\alpha} \mu \bar{\gamma} \rho}^{\mathrm{ph},+} I_{\bar{\beta} \rho \bar{\delta} \mu}^{\mathrm{ph},+}\right. \\
& \left.+I_{\bar{\alpha} \mu \bar{\gamma} \rho}^{\mathrm{ph},-} I_{\bar{\beta} \rho \bar{\delta} \mu}^{\mathrm{ph},-}-I_{\bar{\beta} \mu \bar{\gamma} \rho}^{\mathrm{ph},+} I_{\bar{\alpha} \rho \bar{\delta} \mu}^{\mathrm{ph},+}-I_{\bar{\beta} \mu \bar{\gamma} \rho}^{\mathrm{ph},-} I_{\bar{\alpha} \rho \bar{\delta} \mu}^{\mathrm{ph},-}\right\},
\end{aligned}
$$

with a computational complexity of $\mathcal{O}\left(N^{6}\right)$. In the case of $M<$ $N$, using the replacement in Eq. (99), this reduces to $\mathcal{O}\left(N^{3} N^{3}\right)$ for the calculation of the intermediates and to $\mathcal{O}\left(N^{2} M^{4}\right)$ for the trace. Repeating our argument from Sec. IIF that $M \propto \sqrt{N}$, we expect a scaling of $\mathcal{O}\left(N^{4}\right)$ for two-dimensional systems.

\section{Efficient trace evaluation}

In order to evaluate the temporary products for the flow of the vertex, Eqs. (100)-(102), it is advantageous to rewrite the expression in terms of a matrix product, e.g.,

$$
I_{\mu,(\rho \bar{\gamma} \bar{\delta})}^{\mathrm{c},+}=\sum_{\nu} P_{\mu \nu}^{\Lambda, s}(\Lambda) \Gamma_{\nu,(\rho \bar{\gamma} \bar{\delta})}^{\Lambda},
$$

where we interpret $(\rho \bar{\gamma} \bar{\delta})$ as a single index, because modern generic matrix-matrix multiplication (GEMM) kernels are highly optimized and perform far better than a simple sum. For the cases where we calculate the renormalization of the vertex for all states, this is trivial. Note that for some equations one needs to retain a copy of the vertex with transposed indices to be able to do this. Since our implementation is typically not 


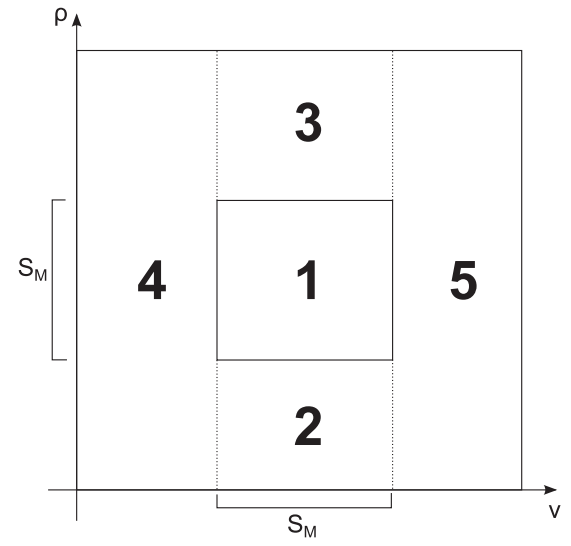

FIG. 3. The subdivision of the GEMM kernel for the intermediate product $I^{\mathrm{c},+}$ in the $v$ and $\rho$ indices. The regions one to five in the diagram correspond to the terms of Eqs. (105)-(109), respectively.

constrained by the available memory but rather the available processing power, this tradeoff is advantageous. It is trickier to approximate the vertex according to Eq. (99). Instead of rewriting the entire expression in terms of a GEMM kernel, we need to perform the loop on the external indices explicitly. We may then split the resulting matrix product into five parts. Taking for example Eq. (100) and using that $\mathcal{H}_{M}$ is the subset of states for which the vertex is renormalized, we have

$$
\begin{aligned}
I_{\mu \rho \bar{\gamma} \bar{\delta}}^{\mathrm{c},+}= & \sum_{\bar{\nu} \in \mathcal{H}_{M}} P_{\mu \bar{\nu}}^{\Lambda, s}(\Lambda) \Gamma_{\bar{\nu} \rho \bar{\gamma} \bar{\delta}}^{\Lambda}\left[\rho \in \mathcal{H}_{M}\right] \\
& +\sum_{\bar{\nu} \in \mathcal{H}_{M}} P_{\mu \bar{\nu}}^{\Lambda, s}(\Lambda) U_{\bar{\nu} \rho \bar{\gamma} \bar{\delta}}\left[\rho<\min \left(\mathcal{H}_{M}\right)\right] \\
& +\sum_{\bar{\nu} \in \mathcal{H}_{M}} P_{\mu \bar{\nu}}^{\Lambda, s}(\Lambda) U_{\bar{\nu} \rho \bar{\gamma} \bar{\delta}}\left[\rho>\max \left(\mathcal{H}_{M}\right)\right] \\
& +\sum_{\nu<\min \left(\mathcal{H}_{M}\right)} P_{\mu \nu}^{\Lambda, s}(\Lambda) U_{\nu \rho \bar{\gamma} \bar{\delta}} \\
& +\sum_{\nu>\max \left(\mathcal{H}_{M}\right)} P_{\mu \nu}^{\Lambda, s}(\Lambda) U_{\nu \rho \bar{\gamma} \bar{s} .}
\end{aligned}
$$

We assume here that the noninteracting states are ordered in energy. The five subexpressions may then be written in terms of GEMM kernels with rectangular blocks of the matrices $P^{\Lambda, s}$ and $U_{\cdot \cdot \bar{\gamma} \bar{\delta}}$. Figure 3 shows the division into these terms in the plane of $v$ and $\rho$ indices. There are no standard kernels for trace evaluation, e.g., Eq. (103), hence we implement that directly in terms of a loop.

\section{Parametrization of the flow equations}

We use an exponential parametrization for the flow equations, Eqs. (31) and (103),

$$
\Lambda=\Lambda_{0} \mathrm{e}^{-l \Delta s}, \quad l \in \mathbb{N},
$$

where $\Lambda_{0}$ is the initial $\Lambda$ at which the flow starts and $l$ is our discretizing iteration number. This parametrization has the advantage that it captures the physics close to the Fermi energy well, as the integration mesh gets denser, while still being relatively fast in reaching that point. Both flow equations are of the form

$$
\frac{d}{d \Lambda} A(\Lambda)=-\frac{1}{2 \pi} B(\Lambda),
$$

allowing for a trivial discretization,

$$
A(\Lambda(l+1))=A(\Lambda(l))+\frac{\Lambda(l) \Delta s}{2 \pi} B(\Lambda(l)),
$$

assuming that $\Delta s$ is sufficiently small. In the following calculations, we have chosen the parameters $\Delta s=0.02$ and $\Lambda_{0}=40$. Unless we encounter a divergence in the flow, we stop as soon as $\Lambda<10^{-4}$ (giving a total of $l_{\max }=645$ iterations).

\section{B. Chemical potential}

We would like to keep the number of particles fixed to study a finite system and its instabilities at a given filling fraction rather than at a given chemical potential. Since our $\Lambda$ flow modifies the real part of the self-energy, we need to constantly adjust the chemical potential during the renormalization procedure. ${ }^{5}$

At $T=0$, we diagonalize the matrix $H_{0}+\Sigma^{\Lambda}$ to obtain the updated quasiparticle energies for a given $\Lambda$ (including the initial $\Lambda_{0}$, since $\Sigma^{\Lambda_{0}} \neq 0$ ). We choose our chemical potential to be

$$
\mu_{\mathrm{chem}}^{\Lambda}=\frac{1}{2}\left(\tilde{\epsilon}_{N_{\mathrm{e}}+1}^{\Lambda}+\tilde{\epsilon}_{N_{\mathrm{e}}}^{\Lambda}\right),
$$

where $\tilde{\epsilon}_{N_{\mathrm{e}}}^{\Lambda}$ is the energy of the highest-occupied quasiparticle state and $\tilde{\epsilon}_{N_{\mathrm{e}}}^{\Lambda}$ the energy of the lowest unoccupied quasiparticle state.

At $T>0$ the value of $\mu_{\mathrm{chem}}^{\Lambda}$ follows as usual from the solution to the equation

$$
N_{\mathrm{e}}=\sum_{\tilde{\epsilon}_{\tilde{\alpha}}^{\Lambda}<\mu_{\text {chem }}^{\Lambda}} n_{\mathrm{F}}\left(\tilde{\epsilon}_{\tilde{\alpha}}-\mu_{\text {chem }}^{\Lambda}\right),
$$

where $N_{\mathrm{e}}$ is the number of electrons and $\tilde{\epsilon}_{\tilde{\alpha}}^{\Lambda}$ are the quasiparticle energies for a given $\Lambda$, i.e., the eigenvalues of $H_{0}+\Sigma^{\Lambda}$.

\section{Correlators}

Starting from Eq. (84), we first transform the vertex into the $\Lambda$-dependent quasiparticle basis,

$$
\tilde{\Gamma}_{\tilde{\mu} \tilde{\nu} \tilde{\rho} \tilde{\sigma}}^{\Lambda}=\sum_{\alpha \beta \gamma \delta} V_{\tilde{\mu} \alpha}^{\mathrm{in}} V_{\tilde{\nu} \beta}^{\mathrm{in}} \Gamma_{\alpha \beta \gamma \delta}^{\Lambda} V_{\gamma \tilde{\rho}}^{\mathrm{in},-1} V_{\delta \tilde{\sigma}}^{\mathrm{in},-1} .
$$

We exploit fast matrix multiplication routines to perform these basis transforms. As these routines require us to group either the three left- or rightmost indices together, we first transform the vertex in $\alpha$ and $\delta$, then transpose it to have $\beta$ as the first

\footnotetext{
${ }^{5}$ The adjustment $\mu(\Lambda)$ gives rise to an additional derivative term in the definition of the single-scale propagator, Eqs. (7) and (25). We neglect the derivative term $d \mu / d \Lambda$ mainly for pragmatic reasons to keep the computational effort feasible. For small system sizes, we have checked the procedure against exact diagonalization. In future work, additional tests could be performed restarting with the chemical potential kept fixed at the value found in the first run.
} 
index and $\gamma$ as the last index, and apply the final pair of transformations, yielding the following sequence of steps:

$$
\begin{aligned}
& \Gamma_{\tilde{\mu} \beta \gamma \delta}^{\Lambda,(1)}=\sum_{\alpha} V_{\tilde{\mu} \alpha}^{\mathrm{in}} \Gamma_{\alpha \beta \gamma \gamma \delta}^{\Lambda}, \\
& \Gamma_{\tilde{\mu} \beta \gamma \tilde{\sigma}}^{\Lambda,(2)}=\sum_{\delta} \Gamma_{\alpha \beta \gamma \delta}^{\Lambda,(1)} V_{\delta \tilde{\sigma}}^{\mathrm{in},-1}, \\
& \Gamma_{\beta \tilde{\mu} \tilde{\sigma} \gamma}^{\Lambda,(3)}=\Gamma_{\tilde{\mu} \beta \gamma \tilde{\sigma}}^{\Lambda,(2)}, \\
& \Gamma_{\tilde{v} \tilde{\mu} \tilde{\sigma} \gamma}^{\Lambda,(4)}=\sum_{\tilde{v}} V_{\tilde{v} \beta}^{\mathrm{in}} \Gamma_{\beta \tilde{\mu} \tilde{\sigma} \gamma}^{\Lambda,(3)}, \\
& \tilde{\Gamma}_{\tilde{\mu} \tilde{\mathcal{v}} \tilde{\rho} \tilde{\sigma}}^{\Lambda}=\sum_{\tilde{\rho}} \Gamma_{\tilde{v} \tilde{\mu} \tilde{\sigma} \gamma}^{\Lambda,(4)} V_{\gamma \tilde{\rho}}^{\mathrm{in},-1} .
\end{aligned}
$$

We do not need to transpose the final result because of the symmetry of $\Gamma$. If our "active space" approximation (ASA) is used, Eq. (99), we employ rectangular submatrices of the $V^{\text {in }}$, since $\Gamma$ is only of size $\mathbb{C}^{M^{4}}$ but $\tilde{\Gamma}$ needs to be of size $\mathbb{C}^{N^{4}}$.

Within ASA a decomposition similar to the one used in the flow equations, Eqs. (105)-(109), is not useful here, as a single matrix multiplication already decomposes into five products. Instead, we transform the entire bare interaction $U$ in the full Hilbert space, and additionally transform $\Gamma-U$ in the activate space and add the results together in the end.

We then proceed to multiply the transformed vertex by the energy denominator of Eq. (84),

$$
\tilde{\Gamma}_{\tilde{\mu} \tilde{\nu} \tilde{\rho} \tilde{\sigma}}^{\Lambda, \text { div }}=\tilde{\Gamma}_{\tilde{\mu} \tilde{v} \tilde{\rho} \tilde{\sigma}}^{\Lambda} \frac{1}{\tilde{\epsilon}_{\tilde{\mu}}+\tilde{\epsilon}_{\tilde{v}}-\tilde{\epsilon}_{\tilde{\rho}}-\tilde{\epsilon}_{\tilde{\sigma}}} .
$$

Finally, we need to transform to the target basis and select the proper orbitals. At $T=0$, we have

$$
\begin{aligned}
& \mathcal{C}_{i \tilde{\nu} \tilde{\sigma}}^{\mathrm{dd}-\operatorname{pre},(2)}=\left[\sum_{\substack{\tilde{\mu} \in \mathcal{H}_{e} \\
\tilde{\rho} \in \mathcal{H}_{h}}}-\sum_{\substack{\tilde{\mu} \in \mathcal{H}_{h} \\
\tilde{\rho} \in \mathcal{H}_{e}}}\right] V_{i \tilde{\mu}}^{\mathrm{ri}} \tilde{\Gamma}_{\tilde{\mu} \tilde{\nu} \tilde{\rho} \tilde{\sigma}}^{\Lambda, \mathrm{div}} V_{\tilde{\rho} i}^{\mathrm{ri} i,-1}, \\
& \mathcal{C}_{i j}^{\mathrm{dd},(2)}=\left[\sum_{\substack{\tilde{\tilde{v}} \in \mathcal{H}_{e} \\
\tilde{\sigma} \in \mathcal{H}_{h}}}-\sum_{\substack{\tilde{\tilde{\tau}} \in \mathcal{H}_{h} \\
\tilde{\sigma} \in \mathcal{H}_{e}}}\right] V_{j \tilde{v}}^{\mathrm{ri}} \mathcal{C}_{i \tilde{\tilde{v}} \tilde{\sigma}}^{\mathrm{dd}-\text { pre,(2) }} V_{\tilde{\sigma} j}^{\mathrm{ri},-1} .
\end{aligned}
$$

Because we transform into the basis of the quasiparticles for a given $\Lambda$, the transformation matrices $V^{\text {in }}$ are $\Lambda$-dependent and the contribution from the bare interaction, $U$, cannot be calculated just once initially. This means that for each $\Lambda$ the density-density correlator incurs a cost of $\mathcal{O}\left(N^{5}\right)$. Equation (121) has a complexity of $\mathcal{O}\left(N^{4}\right)$ and Eq. (122) a complexity of $\mathcal{O}\left(N^{5}\right)$. This cannot be simplified further without additional approximations, making it the most expensive object to calculate.

Fortunately, the density-density-correlator is not actually required for the flow of the vertex or the self-energy. Therefore, unless we see a divergence in our flow in $\Lambda$, we calculate it only once at the very end of the flow. In case a divergence is seen, we perform a backtracking procedure: while we do not store the vertex for all iteration steps, we do keep it for the last $n_{\mathrm{bt}}$ iterations. Once we detect a divergence, we reset the system to the current iteration minus $n_{\mathrm{bt}}$ steps (typically
10) and calculate the density-density correlator at that iteration step and proceed to the next iteration again. This is performed for a total of $n_{\mathrm{dv}} \leqslant n_{\mathrm{bt}}$ iterations (typically 1 or 2 ), where we do not need to recalculate the flow but can just use the known self-energy and the vertex.

\section{VERIFICATION: TESTS ON THE SPINLESS HUBBARD MODEL}

In this section, we test our implementation applying it to disordered spinless Hubbard model. We compare results from $\epsilon$ FRG for the quasiparticle energies and the particle density to the exact diagonalization (ED) in 2D and to the density matrix renormalization group (DMRG) in 1D.

The corresponding Hamiltonian reads

$$
\hat{H}=-t \sum_{\langle i j\rangle} \hat{\mathrm{c}}_{i}^{\dagger} \hat{\mathrm{c}}_{j}+\sum_{i} \delta \epsilon_{i} \hat{\mathrm{n}}_{i}+U \sum_{\langle i j\rangle} \hat{\mathrm{n}}_{i} \hat{\mathrm{n}}_{j},
$$

where $t$ is the hopping parameter, $U$ the interaction strength and the $\delta \epsilon_{i}$ the on-site energies, which are chosen at random from a box distribution with width $W$ centered around $\epsilon=0 .{ }^{6}$ In all calculations we will be working at half-filling. All energies will be measured in units of $t$.

\section{A. $\boldsymbol{\epsilon}$ FRG versus ED for square lattices}

In this section, we test our implementation of the $\epsilon$ FRG equations. To this end, we work with small systems, so ED is feasible and there is no need to apply the ASA. Specifically, we consider the model Hamiltonian of Eq. (124) on a $4 \times 4$ square lattice with $N=16$ sites and periodic boundary conditions at half filling, $v=1 / 2$. The details of our ED-implementation are given in Appendix C.

a. Density. Figure 4 (left) displays the interaction induced shift of the particle density as it is obtained for a typical disorder realization at very weak interactions and disorder $U=0.01$ and $W=0.1$. To highlight the density response, we have divided the relative displacement by $U$. We obtain a checkerboard pattern that we interpret as a precursor to the system ordering in a charge-density wave (CDW). In the absence of disorder, there is a twofold degeneracy associated with the placement of the wave. The pattern is visible in our calculation due to the disorder which breaks this symmetry. As seen from Fig. 4 (right) the density response to very small values of $U$ is reproduced by the $\epsilon \mathrm{FRG}$ reasonably well with a typical error of about $30 \%$.

A comparison at stronger interaction and disorder is given in Fig. 5 where $U=0.1$ and where the disorder potential of the previous realization has been recycled, but multiplied with a factor of fifty corresponding to $W=5.0$.

b. Quasiparticle energies. We also compare the spectral properties, i.e., the quasiparticle energies, for both systems, see Fig. $6{ }^{7}$ The ordinate shows the energies of the corresponding

\footnotetext{
${ }^{6}$ In 2D, this model could be realized in terms of a strongly screened two-dimensional electron gas with a strong in-plane magnetic field. This would polarize all of the spins due to the Zeemann effect but have no orbital contribution.

7. To obtain the quasiparticle energies in the ED case, we calculate the spectral function utilizing the truncated Chebyshev expansion
} 

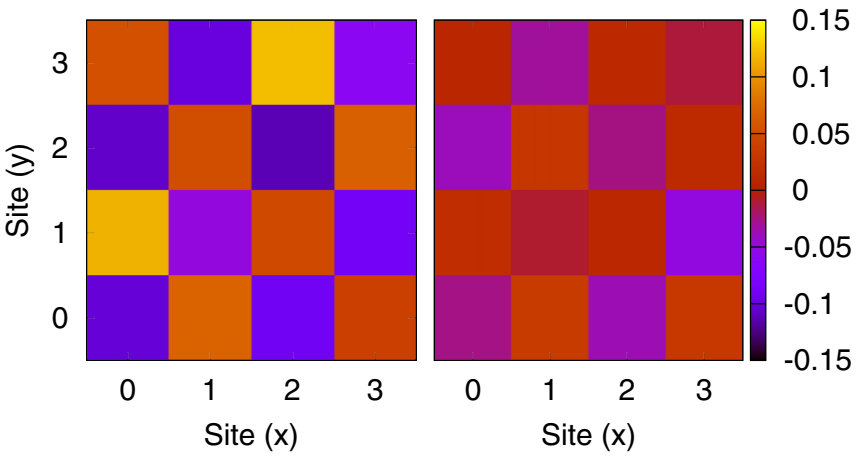

FIG. 4. Comparison of the particle density, $n(\mathbf{r})$, calculated from $\mathrm{ED}\left(n_{\mathrm{ED}}\right)$ and $\epsilon \mathrm{FRG}\left(n_{\epsilon \mathrm{FRG}}\right)$ for a single disorder realization at $U=0.01$ and $W=0.1$. (Left) Normalized relative deviation $\left(n_{\mathrm{ED}}-n_{0}\right) / n_{0} U$, where $n_{0}$ denotes the density for the same disorder realization at $U=0$. (Right) $\left(n_{\mathrm{ED}}-n_{\epsilon \mathrm{FRG}}\right) / n_{0} U$.

noninteracting system, i.e., of $\hat{H}_{0}$. At low disorder, $W=0.1$, the degeneracies of the clean system are only slightly lifted, hence the crosses in Fig. 6 appear in groups. The vertical spreading of these groups is seen to be larger than for the case with stronger disorder, $W=5$. We attribute the larger error for the near-degenerate situation to the fact that our formulation of the $\epsilon$ FRG assumes that $\hat{H}_{0}$ is nondegenerate and becomes singular, otherwise.

We observe that the normalized deviations between $\epsilon$ FRG and ED are approximately independent of the interaction strength $U$. For the occupied states below the chemical potential, $\mu_{\text {chem }} \approx 0$, the error depends very weakly on energy with a typical error smaller than 5\%. In contrast, the deviations keep growing for the unoccupied levels reaching values of $20 \%$ near the band edge.

\section{B. Active space approximation (ASA)}

As has been discussed in Sec. IIF, we will consider the renormalized vertex within an active space of $M<N$ states. In this section, we test the sensitivity of $n(\mathbf{r})$ and the spectral function to variation of $M$. To this end, we will use a $6 \times 6$ square lattice with periodic boundary conditions, so $N=36$. Each system is calculated twice, with the full $M=36$ and with $M=16$.

The real space density at $U=0.01$ for a specific disorder realization at $W=1$ is shown in Fig. 7. We see that there is a very good agreement between the density profiles of both methods, validating our approach at least for small system sizes and moderate interaction strengths.

We also compare the quasiparticle energies as obtained from $\epsilon$ FRG for both choices of $M=36$ and 16. Figure 8 shows the normalized difference of both spectra. As can be seen, the overall performance of ASA is acceptable with a

discussed in Appendix C, where we have kept $10^{5}$ Chebyshev moments. With an artificial broadening $\left(2 \times 10^{-3} t\right)$ to ensure the validity of the truncation of the expansion, the resulting density of states has been fitted against Lorentzians (with a maximum relative error of the position always below $10^{-6} t$ for each peak).
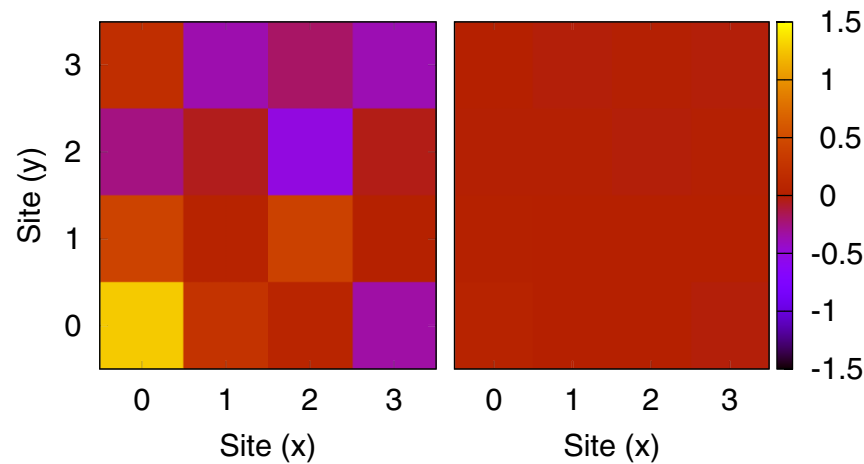

FIG. 5. Plot similar to Fig. 4 with $U=0.1$ and $W=5$.

relative error of about $0.5 \%$ for quasiparticle energies close to the Fermi level. Remarkably, the error does not exceed 1\% even for states outside of the active space.

\section{C. $\boldsymbol{\epsilon}$ FRG versus DMRG for chains}

As a second, independent line of testing we also compare the results from $\epsilon$ FRG with DMRG calculations. To this end we consider the same Hamiltonian (124) as before, but now the geometry represents a short chain of $L=16$ sites. In the $\epsilon$ FRG, we keep $N=16=M$. At a given, fixed disorder configuration with $W=0.2$, we compare the particle density for two different interaction strengths, $U=0.2$ and 1.5.

Figure 9 displays the response of the density when switching on $U$ as obtained with both methods. At smaller interaction values, $U=0.2$, the $\epsilon$ FRG reproduces the DMRG results quantitatively with errors in the percent-regime. When the interaction reaches values of the order of the bandwidth, $2 t$, larger deviations occur reaching values of up to $50 \%$. The systematic overshooting that is observed in the data, we tentatively attribute to a lack of screening related to the static approximation.

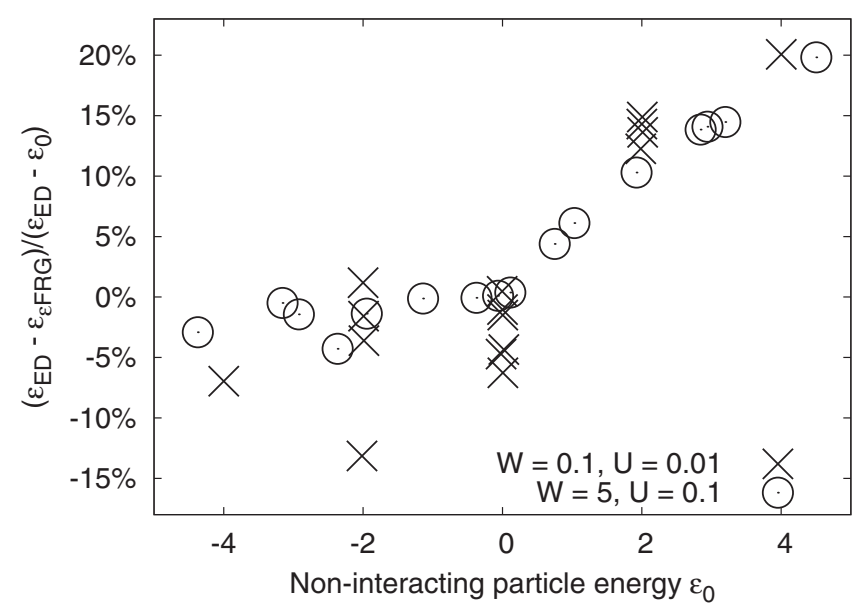

FIG. 6. Comparison of the quasiparticle energies obtained with $\epsilon$ FRG and ED, normalized by the interaction-induced shift, $\left(\epsilon_{\mathrm{ED}}-\epsilon_{\epsilon \mathrm{FRG}}\right) /\left(\epsilon_{\mathrm{ED}}-\epsilon_{0}\right)$, for the same systems as in Figs. 4 (crosses) and 5 (circles), respectively. 


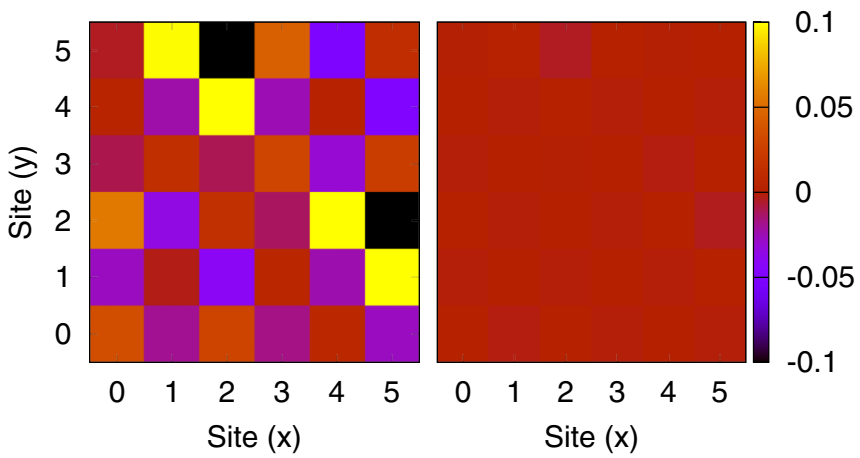

FIG. 7. Testing the ASA via density calculations at $N=36$ with $U=0.1$ for a given disorder realization at $W=1.0$. (Left) $\left(n_{\epsilon \mathrm{FRG}}-n_{0}\right) / U n_{0}$. (Right) $\left(n_{\epsilon \mathrm{FRG}}-n_{\mathrm{ASA}}\right) / U n_{0}$ where $M=16$ has been used in the ASA calculation.

\section{Detecting the CDW state with $\epsilon$ FRG and ASA}

As we pointed out in Sec. IIG, the FRG formalism signalizes the presence of an instability of the Fermi liquid via runaway flow of certain elements of the interaction vertex $\Gamma$. Therefore a matrix norm, e.g.,

$$
\left|\Gamma^{\Lambda}\right|=M^{-4} \sqrt{\sum_{\bar{\alpha} \bar{\beta} \bar{\gamma} \bar{\delta}}\left(\Gamma_{\bar{\alpha} \bar{\beta} \bar{\gamma} \bar{\delta}}^{\Lambda}\right)^{2}},
$$

is a reliable indicator of a nearby instability. Figure 10 shows how this norm flows under the action of the RG. It is seen to diverge, e.g., at $\Lambda_{\mathrm{c}} \approx 0.04$ for $U=0.1$.

Ideally, to pinpoint the nature of the instability, one would investigate which one of the matrix elements of $\Gamma$ diverges so as to predict the nature of the instability. Since we here expect a CDW, we omit this step and just check that this interpretation is indeed consistent with the $\epsilon$ FRG results. At first sight, one might suspect that it would be sufficient to this end calculating the particle density $n(\mathbf{r})$ and ensuring that it indeed exhibits the checkerboard pattern. However, this perspective is slightly misleading. In the presence of runaway

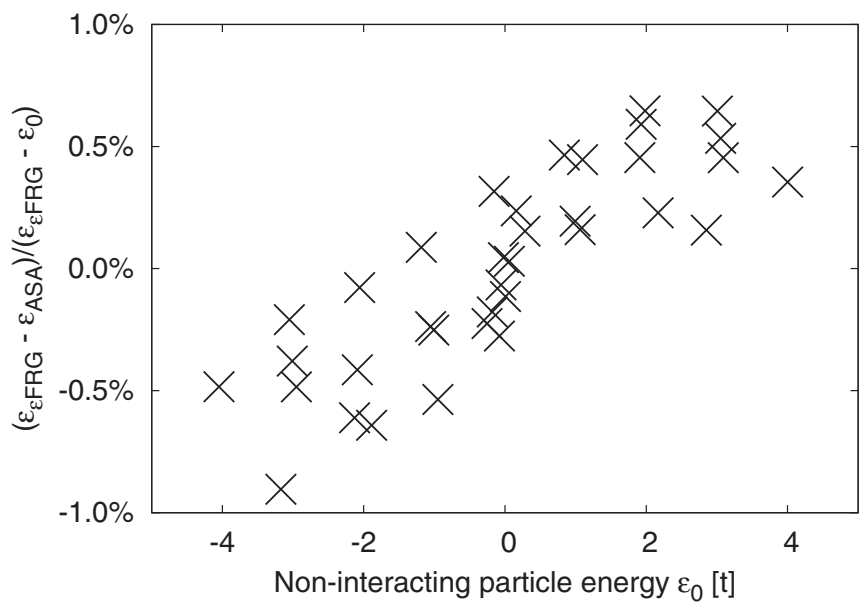

FIG. 8. Difference of the quasiparticle energies obtained with ASA $\left(\epsilon_{\mathrm{ASA}}, M=16\right)$ and without $\left(\epsilon_{\epsilon \mathrm{FRG}}, N=M=36\right)$ normalized by the interaction induced shift: $\left(\epsilon_{\epsilon \mathrm{FRG}}-\epsilon_{\mathrm{ASA}}\right) /\left(\epsilon_{\epsilon \mathrm{FRG}}-\epsilon_{0}\right)$. The same sample was used as in the previous Fig. 7.

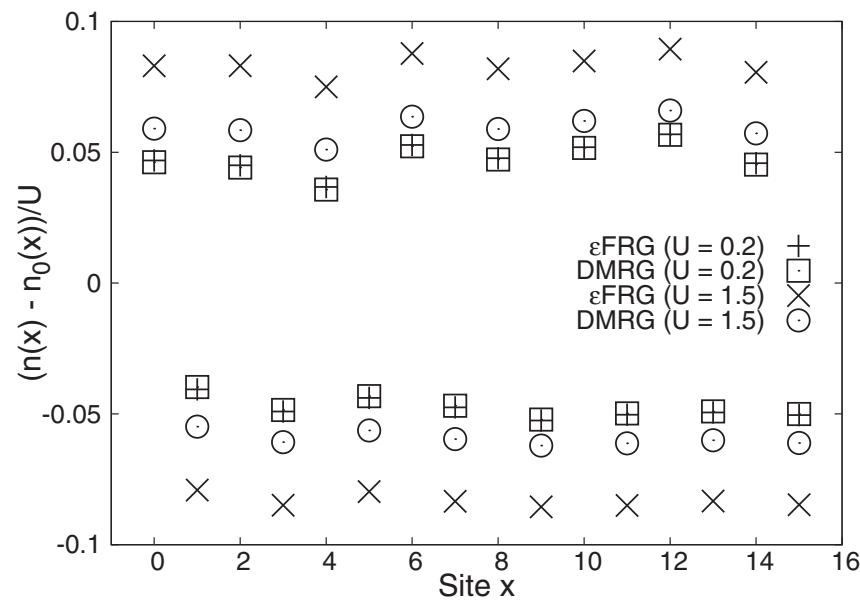

FIG. 9. Normalized interaction induced density response, $\left(n_{\mathrm{X}}-n_{0}\right) / U$, of a 16-site chain obtained with DMRG (empty squares an circles) and FRG (crosses) at $U=0.2$ and $1.5 ; n_{0}$ denotes the noninteracting density.

flow, we cannot evaluate the density at $\Lambda=0$, but only at $\Lambda \gtrsim \Lambda_{c}$ where the ground-state does not yet fully exhibit the broken symmetry. Therefore, instead of calculating $n(\mathbf{r})$, one rather evaluates the density correlator at $\Lambda=\Lambda_{c}$,

$$
\mathcal{D}(\mathbf{k})=N^{-1} \sum_{\mathbf{x x}^{\prime}} \mathrm{e}^{\mathrm{i} \mathbf{k}\left(\mathbf{x}-\mathbf{x}^{\prime}\right)} \mathcal{C}_{i=(x, y), j=\left(x^{\prime}, y^{\prime}\right)}^{\mathrm{dd},(2)},
$$

where $\mathcal{C}^{\mathrm{dd},(2)}$ may be calculated according to Eq. (84). The result for $\mathcal{D}$ is displayed in Fig. 11 (left column) for two different values of interactions and disorder. The peak at the correct ordering wave number $(\mathbf{Q}=\pi, \pi)$ of the density response is already clearly visibly foreshadowing the upcoming ordered phase.

To give further evidence of the correct prediction of charge ordering, we also calculate the real space density. Since due to runaway flow this cannot be done with $\epsilon \mathrm{FRG}$, we again employ the ED. As expected, the resulting densities-shown in Fig. 11 (right column) - exhibit the checkerboard pattern.

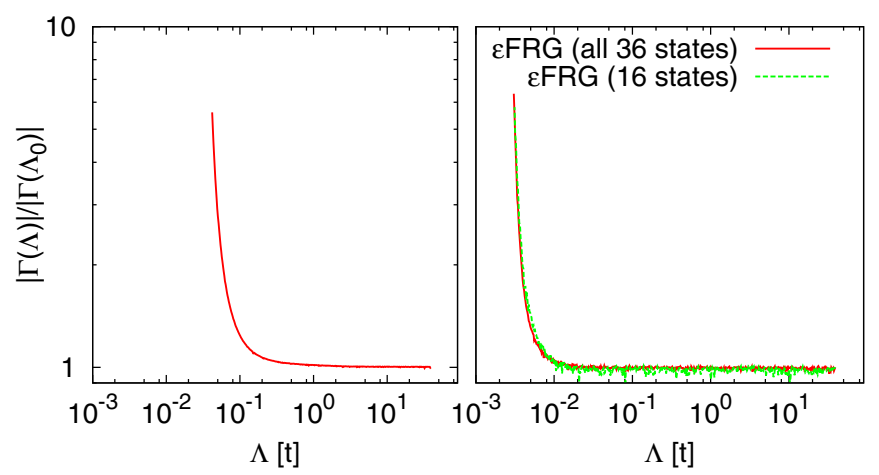

FIG. 10. RG flow of the norm of $\Gamma^{\Lambda}$ for $U=W=0.1$ on a lattice $4 \times 4$ (left) and $U=0.01, W=0.001$, and $N=36$ on a lattice $6 \times 6$ (right). The right panel shows in addition to full $\epsilon \mathrm{FRG}(M=36)$ also ASA data with $M=16$ demonstrating that the critical value $\Lambda_{c}$ is a very robust indicator of runaway flow. 

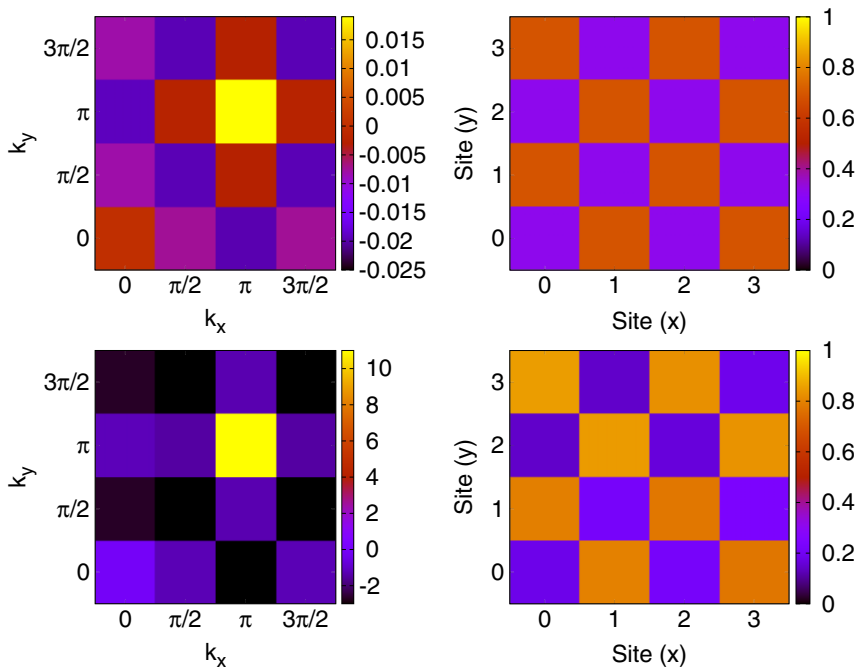

FIG. 11. (Left) The density-density correlator as defined in Eq. (126) calculated at $\Lambda_{\mathrm{c}}$ at $U=W=0.01$ (upper row, system in Fig. 10) and at $U=W=5$ (lower row). The peak indicates the CDW instability with wave vector $(\mathbf{Q}=\pi, \pi)$. (Right) Respective densities $n(\mathbf{r})$ from exact diagonalization (ED) exhibiting the corresponding pinned CDW.

We have already demonstrated that $\Lambda_{c}$ is properly reproduced within ASA. As a final step in this section we show that this is also the case for the density response $\mathcal{D}$. In Fig. 12, we compare two calculations with full $\epsilon \mathrm{FRG}, N=M=36$ and with ASA $(M=16)$ for a system with very weak disorder and interaction. As is seen there, the ordering peak is quantitatively reproduced by the active-space approximation to the $\epsilon \mathrm{FRG}$.

\section{APPLICATION: PHASE DIAGRAM OF SPINLESS DISORDERED HUBBARD MODEL}

As a relevant application of our method, we determine the phase diagram of the spinless Hubbard model on a square lattice with periodic boundary conditions. For two limiting cases the phases of the model are well known. In the absence of disorder, $W=0$, the ground state exhibits the charge-density wave (CDW) at any finite value of $U>0$ [79]; it already made
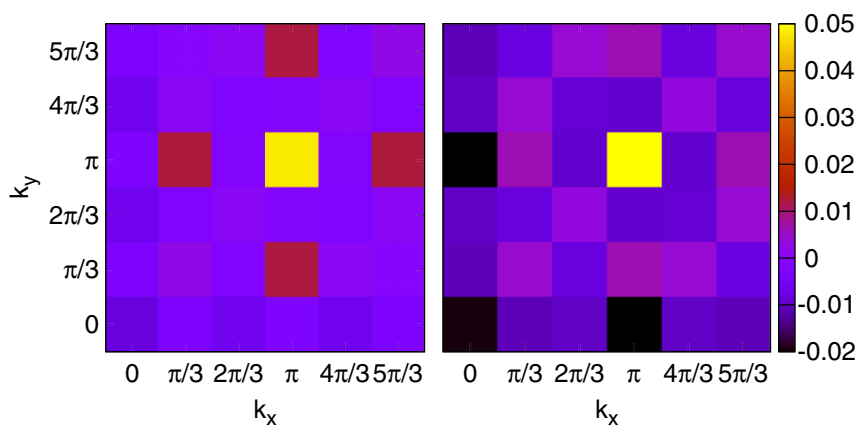

FIG. 12. The density-density correlator as obtained from $\epsilon$ FRG at $\Lambda_{\mathrm{c}}$ with (left, $M=16$ ) and without (right, $N=M=36$ ) ASA for $W=0.001$ and $U=0.01$ on a $6 \times 6$ lattice. The peak is well exposed in both plots, so the CDW nature of the ordering phase is reliably reproduced by ASA. its appearance in the previous section. On the other hand, in the absence of interaction, $U=0$, the system becomes an Anderson insulator (AI) for any finite disorder $W>0$ [80]. The purpose of this investigation is to determine the phaseboundary in the general case, $U$ and $W>0$, as is indicated in the $\epsilon$ FRG by runaway flow.

Our tests on small systems so far have indicated, that with disorder, $W>0$, a minimum value of the interaction, $U^{*}(W)$, is required for the system to form a CDW ground state. This is in contrast to the clean case where for any $U>0$ a charge density order is established, at least for large enough systems. We evaluate $U^{*}(W)$ with the $\epsilon$ FRG.

Note that $U^{*}$ will somewhat vary between different disorder realization and may, in addition, exhibit a dependency on the system size $L$. To deal with this, we apply the following strategy; for a fixed system size and disorder realization, we scan over $U$ and thus obtain $U^{*}$ for this specific sample. We repeat the run for more samples with different disorder realizations keeping the same disorder strength $W$ thus finding the average $U^{*}(W, L)$. Finally, to account for finite size effects we analyze the behavior of $U^{*}(L, W)$ for varying system sizes, $L$.

At this point it should be noted that the available system sizes will allow to study the typical behavior within a range of moderate system sizes. Physics that emerges only at larger length scales will not be seen. This includes, e.g., the effect of rare regions. Similarly, the number of samples constructed for the ensemble average will reveal the typical but may be too small for highlighting also the effect of untypical events.

\section{A. Results: phase diagram}

Figure 13 displays $U^{*}(W, L)$ after averaging over five disorder configurations for $L \times L$ lattices with $L=4,6$, and 8 . For $L=6$ and 8 , we have used ASA with $M=16$ states in both cases. Our data indicates that except at very large disorder values, $W=3, U^{*}$ appears to remain largely insensitive to variations of the (lateral) system size by a factor of two. We take this as an indication that $U^{*}$ will indeed remain finite

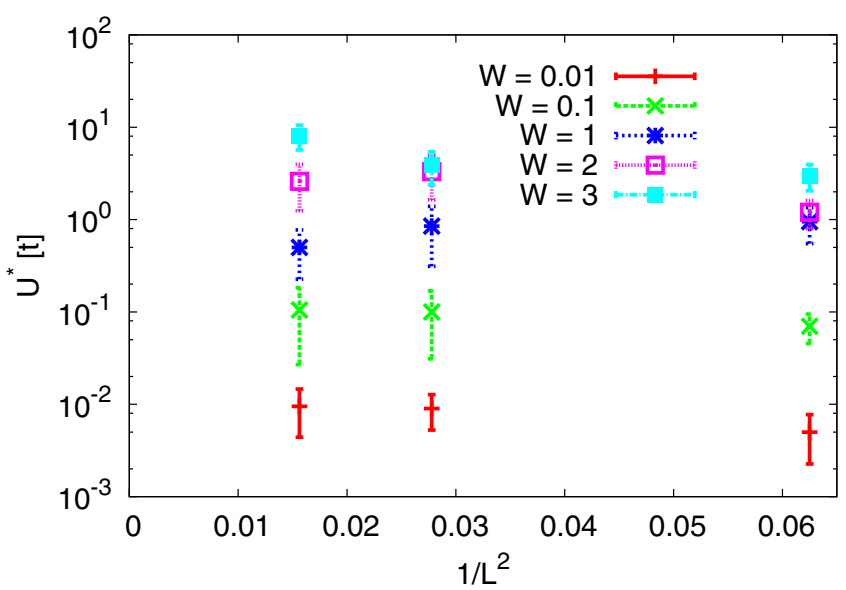

FIG. 13. Critical interaction $U^{*}$ beyond which $\epsilon$ FRG predicts CDW-ordering plotted over the inverse system size $1 / L^{2}$ for multiple different values of the disorder strength $W$. The results have been averaged over five disorder configurations and $1 \sigma$ error bars are given. 


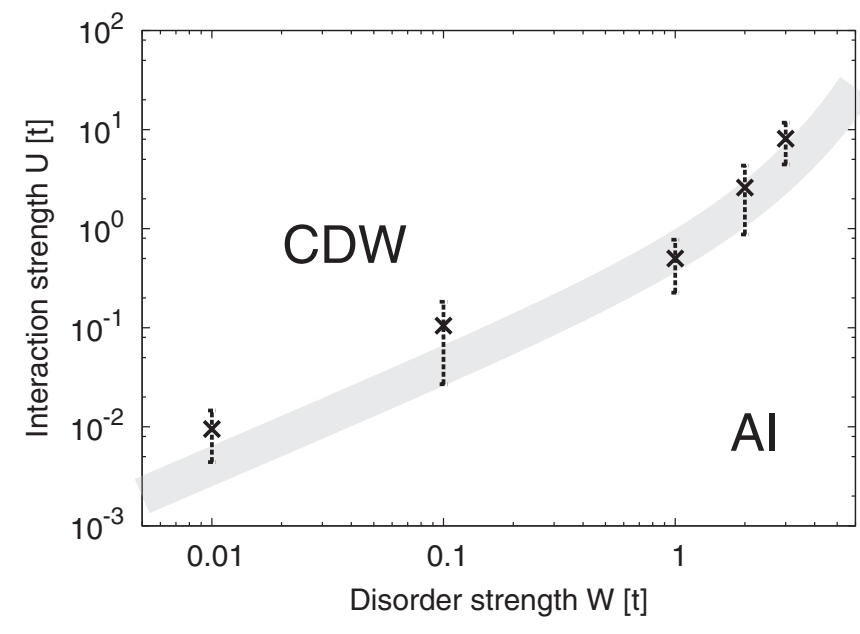

FIG. 14. The phase diagram for the spinless disordered Hubbard model in $2 \mathrm{D}$ as calculated with $\epsilon$ FRG.

even at large system sizes. Thus encouraged we take the data at $L=8$ as an estimate for the phase boundary $U^{*}(W)$ at $L \rightarrow \infty$. Figure 14 shows the resulting phase diagram.

Computational details. We found it practical to work with a single particle Hilbert space containing $N \sim 50-100$ states. For example, with a single-particle Hilbert space consisting of $N=64$ states and the active space consisting of $M=32$ states, a single calculation on eight CPU cores takes less than 24 hours.

\section{B. Discussion}

\section{Stability arguments and quasistatic approximation}

Due to the quasistatic approximation the $\epsilon$ FRG-self-energy is Hermitian and energy-independent. On this level of approximation, the interaction is dealt with by replacing the noninteracting Hamiltonian $H_{0}$ with an effective quasiparticle (qp) Hamiltonian $H_{\mathrm{qp}}$. The latter deviates from $H_{0}$ by a renormalized kinetic energy term, a renormalized effective potential that can, in general, carry off-diagonal entries.

After these preliminaries, one expects that the Andersonlocalized phase (at $U=0$ ) is seen to be stable within quasistatic $\epsilon$ FRG against introducing a small repulsive interaction. ${ }^{8}$ After all, the renormalized Hamiltonian $\hat{H}_{\text {qp }}$ is still a generic representative of the orthogonal symmetry class and hence should exhibit conventional behavior.

A similar stability argument also applies to the ordered phase: the leading effect of weak disorder is pinning of the charge-density wave (CDW). The wave is destroyed only when strong fluctuations of the local potential allow for lattice defects, where two neighboring lattice sites are occupied. For box-distributed on-site potentials, isolated defects can occur only when $W \sim U$. As a consequence, one expects $U^{*}(W) \sim W$ at weak disorder $W$, which is consistent with the phase boundary seen in Fig. 14. Remarkably, at interaction strengths comparable to the bandwidth, $U \gtrsim 1$, the disorder

\footnotetext{
${ }^{8}$ We tacitly assume here that the short-range Hubbard term does not introduce long-range correlations in the matrix elements of $H_{\mathrm{qp}}$.
}

strength necessary to destroy the CDW appears to be considerably smaller than $U$. We hypothesize that we here witness the onset of a collective effect in which several particles can optimize their energy with respect to the disorder potential at the expense of very few particles that built up a defect line thus producing a phase separation.

Physics beyond the quasistatic approximation. The quasistatic approximation ignores the energy exchange between the quasiparticles that of course also is included in the model Hamiltonian, Eq. (124). Effects of dephasing and many-body localization [81] are beyond its scope. Therefore we consider it likely that the phase seen as (conventional) Anderson-insulator by the (quasistatic) $\epsilon \mathrm{FRG}$ is missing aspects of dynamical physics that dominate essential properties of the phase at nonvanishing temperature. What implications this may have on the (zero-temperature) phase-boundary between the CDW and the Anderson insulator remains to be seen.

\section{Relation to earlier work}

The spin-1/2 Hubbard model enjoyed considerable attention in recent years, because physical realizations can be found not only within condensed matter systems but also in cold atomic gases, see Refs. [82,83] for very recent results. In principle, also the spinless model, Eq. (124), that we deal with in this work could find a cold-atom realization which, however, would require the application of a strong homogeneous in-plane magnetic field. This could be one reason, why the spinless model, Eq. (124), has received considerably less attention over the years.

Numerical investigations of the spinless model have been concentrating on its quantum glass variant that deviates from Eq. (124) replacing the short-range interaction by a long-range Coulomb interaction [84-86]. An analytical treatment of the model, Eq. (124), has been given by Vlaming et al., Refs. [87,88]. The authors employed the Bethe lattice where an exact solution can be given in the limit of infinite branching number. The physical picture developed there for the zero temperature limit is in qualitative agreement with our own findings. More recently, Foster and Ludwig studied the model, Eq. (124), with (complex) off-diagonal disorder focussing on the effect of interactions on the Gade fixed point [89]. In that case the noninteracting reference state is not an insulator but a (critical) metal that-according to perturbative $\mathrm{RG}$-is unstable against weak repulsive interactions.

\section{CONCLUSION AND OUTLOOK}

The main purpose of this work was a methodological one: to develop, implement and test a variant of the traditional functional renormalization group (FRG) method that is applicable to generic systems, such as molecules or disordered metal grains, which are lacking translational invariance. Within the new approach $(\epsilon \mathrm{FRG})$, the renormalization of the interaction vertex occurs only for matrix elements with singleparticle states that are situated in an energy shell around the Fermi-energy (active space). The method is computationally efficient provided this shell can be taken smaller than the (noninteracting) bandwidth. We argue that the scaling with the size of the single-particle Hilbert space $N$ should be $N^{4}$ for 
2D-lattice systems, which compares favorably well with the typical $N^{6}$ scaling of competing methods, such as $\operatorname{CCSD}(\mathrm{T})$. Specifically, calculations with $N=64$ and an active space of size $M=32$ require less than 24 hours on eight CPU cores.

An explicit implementation of $\epsilon \mathrm{FRG}$ has been coded for the spinless Hubbard model in 1D and 2D in the presence of on-site disorder. A comparison to (numerically exact) calculations employing the diagonalization of small systems suggests that the accuracy of $\epsilon$ FRG concerning quasiparticle energies typically is below $20 \%$ in relative error to the interaction-induced shift, as compared to the noninteracting system. Similarly, the interaction induced shift in the groundstate density is recovered quantitatively at small interaction strength $U$ with an error that increases to $\sim 50 \%$ if $U$ reaches the bandwidth.

At its current development stage, the $\epsilon \mathrm{FRG}$ is readily applicable to models of interacting fermions in low dimensions, which includes Hubbard clusters with spin and (attractive) interactions at different filling fractions, but also, e.g., small molecules. Our preliminary tests suggests that with the current formalism system sizes of, e.g., $N=256$ are already within reach. Significantly bigger system sizes might be attainable, after additional improvements in the code performance have been implemented. As an example we mention the numerical integration of the flow equations that at present is done in the simplest possible discretization scheme. Also, the flow equations are well-suited for parallelization on distributed memory systems, allowing for a significant increase in the number of CPU cores used in a single calculation. To give a perspective, we mention that the molecules in the GW100 test set have been described with a QZVP basis set requiring approximately 800 basis function for the biggest species, the amino-acids Guanin and Adenin [4].

We hope that this work helps paving the way for electronic-structure calculations beyond the present paradigm of $G W$-BSE. Admittedly before the envisioned applications to real systems, an efficient $\epsilon$ FRGimplementation should be installed that is also prepared for dealing with long-range interactions. Here, we see at present the biggest bottleneck to be overcome in future research. Perhaps additional motivation to overcome this obstacle could come from the fact that we have also given formulae for the finite-temperature formalism in this work, so that the effect of heat could be included.

\section{ACKNOWLEDGMENTS}

We thank S. Bera, A. D. Mirlin, J. Reuther, J. Schmalian, M. van Setten, and P. Wölfle for inspiring discussions. We are indebted to A. D. Mirlin for supporting our project in an early stage. Support has also been received from the DFG under grants EV30/7-1, EV30/11-1, and EV30/12-1 and from the Landesgraduiertenförderung of the state of Baden-Württemberg. The DMRG results shown here have been provided by F. Weiner using the Schmitteckert code. We acknowledge the support provided by computational resources of the Institute of Nanotechnology (INT) and the Steinbuch Centre for Computing (SCC), both at the Karlsruhe Institute of Technology (KIT).

\section{APPENDIX A: FLOW EQUATIONS FOR $\Gamma$ IN THE STATIC LIMIT}

Here we will derive the flow equation for $\Gamma$ in the static limit, Eq. (32), analogous to the derivation for the self-energy. Starting at Eq. (21), looking at the first term,

$$
\int d \bar{\omega} \sum_{\mu \nu \rho \sigma} \mathcal{G}_{\rho \mu}^{\Lambda}(\bar{\omega}) \mathcal{S}_{\sigma \nu}^{\Lambda}(-\bar{\omega}) \Gamma_{\alpha \beta \rho \sigma}^{\Lambda} \Gamma_{\mu \nu \gamma \delta}^{\Lambda},
$$

it can be seen that by exchanging all traced indices in both vertices that appear, and then renaming the summation indices, the formula may be rewritten as

$$
\int d \bar{\omega} \sum_{\mu \nu \rho \sigma} \mathcal{S}_{\rho \mu}^{\Lambda}(-\bar{\omega}) \mathcal{G}_{\sigma \nu}^{\Lambda}(\bar{\omega}) \Gamma_{\alpha \beta \rho \sigma}^{\Lambda} \Gamma_{\mu \nu \gamma \delta}^{\Lambda},
$$

which is just an exchange of both propagators. Utilizing this, we may write it formulated in terms of matrix products,

$$
\frac{1}{2} \operatorname{tr} \int d \bar{\omega}\left[\mathcal{S}^{\prime} \Gamma^{\mathrm{T}} \mathcal{G}^{\mathrm{T}} \Gamma+\mathcal{G} \Gamma^{\mathrm{T}} \mathcal{S}^{\prime \mathrm{T}} \Gamma\right] .
$$

We note that the frequency of the single-scale propagator is negative here, which we denote with prime for $\mathcal{Q}$ and $\Sigma$; the $\Theta$ and $\delta$ functions only depend on the modulus. Inserting Eq. (26) and using the same representation for $\mathcal{G}$, we can separate four terms,

$$
\begin{aligned}
& -\frac{\delta}{2} \frac{1}{\mathcal{Q}^{\prime}-\Theta \Sigma^{\prime}} \Gamma^{\mathrm{T}}\left(\frac{\Theta}{\mathcal{Q}-\Theta \Sigma}\right)^{\mathrm{T}} \Gamma, \\
& -\frac{\delta}{2} \frac{\Theta}{\mathcal{Q}-\Theta \Sigma} \Gamma^{\mathrm{T}}\left(\frac{1}{\mathcal{Q}^{\prime}-\Theta \Sigma^{\prime}}\right)^{\mathrm{T}} \Gamma, \\
& -\frac{\delta}{2} \frac{1}{\mathcal{Q}^{\prime}-\Theta \Sigma^{\prime}} \Sigma^{\prime} \frac{\Theta}{\mathcal{Q}^{\prime}-\Theta \Sigma^{\prime}} \Gamma^{\mathrm{T}}\left(\frac{\Theta}{\mathcal{Q}-\Theta \Sigma}\right)^{\mathrm{T}} \Gamma, \\
& -\frac{\delta}{2} \frac{\Theta}{\mathcal{Q}-\Theta \Sigma} \Gamma^{\mathrm{T}}\left(\frac{\Theta}{\mathcal{Q}^{\prime}-\Theta \Sigma^{\prime}}\right)^{\mathrm{T}} \Sigma^{\prime \mathrm{T}}\left(\frac{1}{\mathcal{Q}^{\prime}-\Theta \Sigma^{\prime}}\right)^{\mathrm{T}} \Gamma .
\end{aligned}
$$

Since all of these terms occur underneath an integral over $\int d \bar{\omega} \delta(|\omega|-\Lambda)$, we may switch primes within each term, and we note for future use that the terms of Eqs. (A1) and (A2) are equal to each other.

We now apply Morris's Lemma again. In both other terms, Eqs. (A3) and (A4), we can rewrite them in terms of derivatives with respect to (w.r.t.) the integration variable $t$,

$$
\begin{aligned}
& -\frac{\delta}{2} \int_{0}^{1} t^{2}\left(\frac{d}{d t} \frac{1}{\mathcal{Q}^{\prime}-t \Sigma^{\prime}}\right) \Gamma^{\mathrm{T}}\left(\frac{1}{\mathcal{Q}-t \Sigma}\right)^{\mathrm{T}} \Gamma d t, \\
& -\frac{\delta}{2} \int_{0}^{1} t^{2} \frac{1}{\mathcal{Q}-t \Sigma} \Gamma^{\mathrm{T}}\left(\frac{d}{d t} \frac{1}{\mathcal{Q}^{\prime}-t \Sigma^{\prime}}\right)^{\mathrm{T}} \Gamma d t .
\end{aligned}
$$

Partial integration of Eq. (A5) yields

$$
\begin{aligned}
& -\frac{\delta}{2}\left[t^{2} \frac{1}{\mathcal{Q}^{\prime}-t \Sigma^{\prime}} \Gamma^{\mathrm{T}}\left(\frac{1}{\mathcal{Q}-t \Sigma}\right)^{\mathrm{T}} \Gamma\right]_{0}^{1} \\
& \quad+\frac{\delta}{2} \int_{0}^{1} 2 t \frac{1}{\mathcal{Q}^{\prime}-t \Sigma^{\prime}} \Gamma^{\mathrm{T}}\left(\frac{1}{\mathcal{Q}-t \Sigma}\right)^{\mathrm{T}} \Gamma d t \\
& \quad+\frac{\delta}{2} \int_{0}^{1} t^{2} \frac{1}{\mathcal{Q}-t \Sigma} \Gamma^{\mathrm{T}}\left(\frac{d}{d t} \frac{1}{\mathcal{Q}^{\prime}-t \Sigma^{\prime}}\right)^{\mathrm{T}} \Gamma d t .
\end{aligned}
$$


One sees that the second term cancels Eqs. (A1) and (A2) and the third term cancels Eq. (A6), leaving the result

$$
-\frac{\delta}{2} \frac{1}{\mathcal{Q}^{\prime}-\Sigma^{\prime}} \Gamma^{\mathrm{T}}\left(\frac{1}{\mathcal{Q}-\Sigma}\right)^{\mathrm{T}} \Gamma,
$$

which can be rewritten in terms of the index notation as

$$
-\frac{1}{2} \sum_{\bar{\omega}= \pm \Lambda} \sum_{\mu \nu \rho \sigma} P_{\rho \mu}^{\Lambda}(-\bar{\omega}) P_{\sigma \nu}^{\Lambda}(\bar{\omega}) \Gamma_{\alpha \beta \rho \sigma}^{\Lambda} \Gamma_{\mu \nu \gamma \delta}^{\Lambda} .
$$

We note that if one were to keep the frequency dependence of the vertex and the self-energy, two cases need to be distinguished: for the case where all external frequencies are zero, the same derivation applies, so our result holds there. For the case where at least some external frequencies are nonzero, the arguments for the $\delta$ and $\Theta$ functions differ, so one may directly insert Eq. (29) into the flow equations for the vertex.

An analogous treatment is possible for the other four terms in Eq. (21). The other terms may be written as

$$
\operatorname{tr} \int d \bar{\omega}\left[\mathcal{S} \Gamma_{\alpha \cdot \delta \cdot} \mathcal{G} \Gamma_{\beta \cdot \gamma \cdot}+\mathcal{G} \Gamma_{\alpha \cdot \delta \cdot} \mathcal{S} \Gamma_{\beta \cdot \gamma \cdot}-[\alpha \leftrightarrow \beta]\right] .
$$

Looking at the first two terms, they may be divided in the same mannger as in Eqs. (A1)-(A4), without the factor $1 / 2$, and with the same frequency for the single-scale and the regular propagator. This yields the result

$$
-\sum_{\bar{\omega}= \pm \Lambda} \sum_{\mu \nu \rho \sigma} P_{\rho \mu}^{\Lambda}(\bar{\omega}) P_{\sigma \nu}^{\Lambda}(\bar{\omega}) \Gamma_{\beta \nu \gamma \rho}^{\Lambda} \Gamma_{\alpha \mu \delta \sigma}^{\Lambda}+[\alpha \leftrightarrow \beta] .
$$

Putting this all together, one arrives at Eq. (32).

\section{APPENDIX B: IMPLEMENTATION DETAILS}

\section{Chemical potential for $T>0$}

Our algorithm to solve this equation for $\mu_{\text {chem }}$ works in three stages: obtain an initial guess for $\mu_{\text {chem }}, \mu_{\text {chem }}^{(0)}$, (trivially) obtain a second guess, $\mu_{\text {chem }}^{(1)}$, with $\operatorname{sgn}\left(N_{\mathrm{e}}\left(\mu_{\text {chem }}^{\Lambda,(1)}\right)-N_{\mathrm{e}}\right)=$ $-\operatorname{sgn}\left(N_{\mathrm{e}}\left(\mu_{\mathrm{chem}}^{\Lambda,(0)}\right)-N_{\mathrm{e}}\right)$ and then use the secant algorithm [90] to iteratively find the final $\mu_{\text {chem }}$.

The initial guess is taken to be the same as for $T=0$, Eq. (113), since at low temperatures the value is a very good approximation. We then calculate

$$
\mu_{\mathrm{chem}}^{\Lambda,(0)}+\operatorname{sgn}\left(N_{\mathrm{e}}\left(\mu_{\mathrm{chem}}^{(0)}\right)-N_{\mathrm{e}}\right) \frac{\Delta}{4} i,
$$

where $\Delta$ is the mean level spacing of the system and $i$ is an integer that starts at 1 and is incremented until the condition $\operatorname{sgn}\left(N_{\mathrm{e}}\left(\mu_{\text {chem }}^{(1)}\right)-N_{\mathrm{e}}\right)=-\operatorname{sgn}\left(N_{\mathrm{e}}\left(\mu_{\text {chem }}^{(0)}\right)-N_{\mathrm{e}}\right)$ is satisfied. In practice $i=1$ or $i=2$ will already be sufficient, which is why $\Delta / 4$ is a good empirical choice here. ${ }^{9}$

Both initial guesses are then used as input for the secant algorithm. Since $N_{\mathrm{e}}(\epsilon)$ is monotonous and the value searched for is encompassed with both guesses, convergence will be quite fast (10 to 20 iterations in practice). We consider the

\footnotetext{
${ }^{9} \mathrm{We}$ cut this scheme off at $i=10$, since it is only used to accelerate the convergence of the secant algorithm, which is likely to also work if the second value does not satisfy the condition, albeit more slowly.
}

chemical potential to be converged if the relative error of the number of electrons,

$$
\left|\frac{N_{\mathrm{e}}\left(\mu_{\text {chem }}^{(i)}\right)-N_{\mathrm{e}}}{N_{\mathrm{e}}\left(\mu_{\text {chem }}^{(i)}\right)-N_{\mathrm{e}}}\right|,
$$

is larger than the square root of the machine precision. While the smallest possible error here would be of the order of $\hat{\epsilon} N$, with $\hat{\epsilon}$ being the machine precision and $N$ the number of orbitals in the system, the energies $\tilde{\epsilon}_{\tilde{\alpha}}$ only have a precision of $\sqrt{\hat{\epsilon}}$ due to the diagonalization procedure.

\section{Parallelization}

We will now discuss how we exploit parallelization in our implementation. We use a scheme based on a shared memory architecture, OPENMP [78]. It is in principle possible to utilize distributed memory methods, such as MPI (Message Passing Interface, [91]), which allow the usage of far more processor cores for the same calculation.

The intermediate products offer a trivial way to parallelize: it is possible to use a parallel version of the GEMM kernel to calculate the matrix products. In the case we track the renormalization of the entire vertex, this would likely be the most efficient avenue. In our case, however, the effective matrix size that is fed into the GEMM kernel is relatively small (we want to calculate the vertex for as few states as possible), so it is unlikely that using a parallel matrix product kernel will scale well even for a low amount of processors. Instead, we parallelize the loops over the two outer indices in the intermediate products and perform serialized matrix products on each processor. This is trivially possible, since the calculations are independent of each other for any given pair of external indices. Similarly, for the evaluation of the trace, we parallelize the loops over all four external indices and have each processor evaluate the trace for a given set of external indices serially.

\section{Restarting}

Calculations for larger systems may take a relatively long time. In case of technical difficulties, we implement a restarting procedure that allows us to continue a calculation at the point where it last stopped. We save the initial $\Lambda$, the step size, the number of selected states $M$, the chosen target $\Lambda$. Furthermore, we keep the last self-energy and vertex as well as the number of the last iteration to complete. These quantities suffice to reproduce the calculation at a later point in time.

\section{APPENDIX C: ED IMPLEMENTATION}

In Sec. IV A, we compare the FRG to exact diagonalization. In the following, we provide edtails on how we implemented ED as a reference method. In our implementation, we construct the full $N_{\mathrm{e}}$-particle Hilbert space. Its dimension is $\left(\begin{array}{l}N \\ N_{\mathrm{e}}\end{array}\right)$ and grows exponentially with the number of orbitals $N$. We systematically construct the basis states of that space and implement the action of the full many-body Hamiltonian on that basis (we do not explicitly construct the matrix elements of the Hamiltonian itself). An iterative eigensolver for sparse problems is employed to calculate the full many-body ground 
state for a given system. We utilize the standard ARPACK package [92] in direct mode. ${ }^{10}$

For simple observables, such as the density, we may then simply calculate expectation values with respect to the manybody ground state,

$$
\left\langle\hat{\mathrm{n}}_{i}\right\rangle=\left\langle 0\left|\hat{\mathrm{c}}_{i}^{\dagger} \hat{\mathrm{c}}_{i}\right| 0\right\rangle .
$$

We also want to calculate the single-particle density of states, $\rho(\epsilon)$. This is given by the expectation value

$$
\begin{aligned}
\rho(\epsilon)= & -\frac{1}{\pi} \Im \operatorname{tr}_{i j}\left\langle\hat{\mathrm{c}}_{i} \frac{1}{\epsilon-\hat{H}+E_{0}+i \eta} \hat{\mathrm{c}}_{j}^{\dagger}\right\rangle \\
& +\left\langle\hat{\mathrm{c}}_{j}^{\dagger} \frac{1}{\epsilon+\hat{H}-E_{0}+i \eta} \hat{\mathrm{c}}_{i}\right\rangle,
\end{aligned}
$$

which we arrive at by Fourier transforming the definition of the retarded Green's function. This expressions contains the inverse of a very large matrix, which needs to be done for every single energy at which the density of states is to be evaluated at. Furthermore, directly inverting such a large matrix is only possible using iterative algorithms, which would again have to be applied for every single energy. We therefore follow an alternative approach as outlined in the $\mathrm{PhD}$ thesis of Alexander Braun [93]. One may expand the denominator in terms of Chebyshev polynomials $T_{n}(x)$, such that we get

$$
\begin{aligned}
& c_{i j, n}^{(+)}=\left\langle 0\left|\hat{\mathrm{c}}_{i} T_{n}\left(a\left(\hat{H}-E_{0}-b\right)\right) \hat{\mathrm{c}}_{j}^{\dagger}\right| 0\right\rangle, \\
& c_{i j, n}^{(-)}=\left\langle 0\left|\hat{\mathrm{c}}_{i}^{\dagger} T_{n}\left(a\left(\hat{H}-E_{0}-b\right)\right) \hat{\mathrm{c}}_{j}\right| 0\right\rangle,
\end{aligned}
$$

where $E_{0}$ is the ground-state energy. The variables $a$ and $b$ are scaling factors that arise due to the fact that the Chebyshev polynomials are only well-defined in the interval $[-1,1]$, so the Hamiltonian needs to be scaled to fit into that range. We

\footnotetext{
${ }^{10}$ The shift-inverse mode is not required, since the eigenvalues we are interested in are taken from the spectrum edges, not the center.
}

note that since we are calculating expectations in the Hilbert spaces for $N_{\mathrm{e}}+1$ and $N_{\mathrm{e}}-1$ particles, we need to take into account the extremal eigenvalues of the Hamiltonian in those spaces. To make sure we don't suffer from numerical artifacts, we scale the argument of the Chebyshev polynomials into the interval $[-0.9,0.9] .^{11}$ This gives us

$$
\begin{aligned}
& \delta=0.1, \quad \text { (distance to interval boundaries }), \\
& a=\frac{2(1-\delta)}{\left(\epsilon_{\max }-E_{0}\right)-\left(\epsilon_{\min }-E_{0}\right)}, \\
& b=\frac{\left(\epsilon_{\max }-E_{0}\right)+\left(\epsilon_{\min }-E_{0}\right)}{2}-\delta,
\end{aligned}
$$

where $\epsilon_{\min \text { max }}$ are the extremal many-body eigenvalues of the system with $N_{\mathrm{e}}+1\left(N_{\mathrm{e}}-1\right)$ particles and $E_{0}$ is the ground state energy for $N_{\mathrm{e}}$ particles.

We may then rewrite the single-particle retarded Green's function in terms of these coefficients,

$$
\begin{aligned}
\mathcal{G}_{i j}(\omega)= & a \sum_{n=0}^{\infty}\left[\alpha_{n}^{+}(a(\omega+i \eta \mp b)) c_{i j, n}^{(+)}\right. \\
& \left.-\alpha_{n}^{-}(a(\omega+i \eta \mp b)) c_{j i, n}^{(-)}\right] .
\end{aligned}
$$

The density of states is then given by the imaginary part of this expression traced over the real space indices, which is why we only need to calculate the diagonal part of this expression. If we terminate the expansion at a finite $n$, the formula remains only valid for finite $\eta$, with

$$
\eta \gtrsim \frac{1}{a n_{\max }} .
$$

For further discussion on this topic, we would like to defer to Alexander Braun's thesis [93].

\footnotetext{
${ }^{11}$ Using exactly $[-1,1]$ does not work, since the polynomials are fixed at the boundaries of the interval. One needs to distance oneself at least by relative error in the eigenvalues from the boundary.
}

[1] F. Bechstedt, Many-Body Approach to Electronic Excitations, Springer Series in Solid-State Sciences Vol. 181 (Springer, Berlin, Heidelberg, 2015).

[2] L. Hedin, Phys. Rev. 139, A796 (1965).

[3] G. Onida, L. Reining, and A. Rubio, Rev. Mod. Phys. 74, 601 (2002).

[4] M. J. van Setten, F. Caruso, S. Sharifzadeh, X. Ren, M. Scheffler, R. Liu, J. Lischner, L. Lin, J. R. Deslippe, S. G. Louie, C. Yang, F. Weigend, J. B. Neaton, F. Evers, and P. Rinke, J. Chem. Theory Comp. 11, 5665 (2015).

[5] W. Metzner, M. Salmhofer, C. Honerkamp, V. Meden, and K. Schoenhammer, Rev. Mod. Phys. 84, 299 (2012).

[6] M. Salmhofer, Renormalization: An Introduction (Springer, Berlin, Heidelberg, 1999).

[7] C. Honerkamp and M. Salmhofer, Phys. Rev. B 67, 174504 (2003).

[8] S. P. Ridgway and C. A. Hooley, Phys. Rev. Lett. 114, 226404 (2015).
[9] C. Bauer, A. Rückriegel, A. Sharma, and P. Kopietz, Phys. Rev. B 92, 121409 (2015).

[10] J. Reuther and P. Wölfle, Phys. Rev. B 81, 144410 (2010).

[11] J. Reuther and R. Thomale, Phys. Rev. B 83, 024402 (2011).

[12] J. Reuther and R. Thomale, Phys. Rev. B 89, 024412 (2014).

[13] C. J. Halboth and W. Metzner, Phys. Rev. B 61, 7364 (2000).

[14] J. Reiss, D. Rohe, and W. Metzner, Phys. Rev. B 75, 075110 (2007).

[15] C. J. Halboth and W. Metzner, Phys. Rev. Lett. 85, 5162 (2000).

[16] R. Gersch, C. Honerkamp, and W. Metzner, New J. Phys. 10, 045003 (2008).

[17] A. A. Katanin, Phys. Rev. B 79, 235119 (2009).

[18] D. D. Scherer, M. M. Scherer, and C. Honerkamp, Phys. Rev. B 92, 155137 (2015).

[19] A. Eberlein, Phys. Rev. B 90, 115125 (2014).

[20] S. Andergassen, T. Enss, V. Meden, W. Metzner, U. Schollwöck, and K. Schönhammer, Phys. Rev. B 70, 075102 (2004). 
[21] S. Streib, A. Isidori, and P. Kopietz, Phys. Rev. B 87, 201107 (2013).

[22] J. Reuther, P. Wölfle, R. Darradi, W. Brenig, M. Arlego, and J. Richter, Phys. Rev. B 83, 064416 (2011).

[23] Y. Iqbal, R. Thomale, F. P. Toldin, S. Rachel, and J. Reuther, arXiv:1604.03438v1.

[24] R. Suttner, C. Platt, J. Reuther, and R. Thomale, Phys. Rev. B 89, 020408 (2014).

[25] L. Janssen and I. F. Herbut, Phys. Rev. B 89, 205403 (2014).

[26] J. Lee, P. Strack, and S. Sachdev, Phys. Rev. B 87, 045104 (2013).

[27] L. Classen, I. F. Herbut, L. Janssen, and M. M. Scherer, Phys. Rev. B 93, 125119 (2016).

[28] S. Friederich, H. C. Krahl, and C. Wetterich, Phys. Rev. B 83, 155125 (2011).

[29] A. Eberlein and W. Metzner, Phys. Rev. B 89, 035126 (2014).

[30] Y. Tanizaki, G. Fejős, and T. Hatsuda, Prog. Theor. Exp. Phys. (2014) 043I01.

[31] H. Yamase, A. Eberlein, and W. Metzner, Phys. Rev. Lett. 116, 096402 (2016).

[32] C. Platt, W. Hanke, and R. Thomale, Adv. Phys. 62, 453 (2013).

[33] S. Andergassen, T. Enss, V. Meden, W. Metzner, U. Schollwöck, and K. Schönhammer, Phys. Rev. B 73, 045125 (2006).

[34] V. Meden, S. Andergassen, T. Enss, H. Schoeller, and K. Schönhammer, N. Jour. Phys. 10, 045012 (2008).

[35] C. Karrasch, R. Hedden, R. Peters, T. Pruschke, K. Schönhammer, and V. Meden, J. Phys.: Condens. Matter 20, 345205 (2008).

[36] F. Bauer, J. Heyder, E. Schubert, D. Borowsky, D. Taubert, B. Bruognolo, D. Schuh, W. Wegscheider, J. von Delft, and S. Ludwig, Nature (London) 501, 73 (2013).

[37] F. Bauer, J. Heyder, and J. von Delft, Phys. Rev. B 89, 045128 (2014).

[38] M. Kinza, J. Ortloff, and C. Honerkamp, Phys. Rev. B 82, 155430 (2010).

[39] A. Katanin, Phys. Rev. B 88, 241401 (2013).

[40] S. Dusuel and D. Zanchi, Phys. Rev. Lett. 93, 206401 (2004).

[41] C. Karrasch and J. E. Moore, Phys. Rev. B 92, 115108 (2015).

[42] F. Bruneval and M. A. L. Marques, J. Chem. Theory Comput. 9, 324 (2013).

[43] S. Körbel, P. Boulanger, I. Duchemin, X. Blase, M. A. L. Marques, and S. Botti, J. Chem. Theory Comput. 10, 3934 (2014).

[44] C. Rostgaard, K. W. Jacobsen, and K. S. Thygesen, Phys. Rev. B 81, 085103 (2010).

[45] J. W. Knight, X. Wang, L. Gallandi, O. Dolgounitcheva, X. Ren, J. V. Ortiz, P. Rinke, T. Körzdörfer, and N. Marom, J. Chem. Theory Comput. 12, 615 (2016).

[46] F. Kaplan, M. Harding, C. Seiler, F. Weigend, F. Evers, and M. van Setten, to appear J. Chem. Theory Comput. 12, 2528 (2016).

[47] L. Kronik, T. Stein, S. Refaely-Abramson, and R. Baer, J. Chem. Theory Comput. 8, 1515 (2012).

[48] S. Refaely-Abramson, M. Jain, S. Sharifzadeh, J. B. Neaton, and L. Kronik, Phys. Rev. B 92, 081204 (2015).

[49] C. Faber, P. Boulanger, C. Attaccalite, I. Duchemin, and X. Blase, Phil. Trans. Roy. Soc. A 372, 20130271 (2013).

[50] F. Bruneval, S. M. Hamed, and J. B. Neaton, J. Chem. Phys. 142, 244101 (2015).
[51] D. Hirose, Y. Noguchi, and O. Sugino, Phys. Rev. B 91, 205111 (2015).

[52] C. Friedrich, S. Blügel, and A. Schindlmayr, Phys. Rev. B 81, 125102 (2010).

[53] http://perso.neel.cnrs.fr/xavier.blase/fiesta/index.html.

[54] J. Deslippe, G. Samsonidze, D. A. Strubbe, M. Jain, M. L. Cohen, and S. G. Louie, Comput. Phys. Commun. 183, 1269 (2012).

[55] http://www.vasp.at.

[56] F. Bruneval, http://github.co/bruneval/molgw.

[57] C. Honerkamp, Phys. Rev. B 85, 195129 (2012).

[58] M. Kinza and C. Honerkamp, Phys. Rev. B 92, 045113 (2015).

[59] F. Evers and A. D. Mirlin, Rev. Mod. Phys. 80, 1355 (2008).

[60] F. Bechstedt, Many-Body Approach to Electronic Excitations, Springer Series in Solid-State Sciences Vol. 181 (Springer, Berlin, Heidelberg, 2015).

[61] F. G. Giuliani and F. Vignale, Quantum Theory of the Electron Liquid (Cambridge University Press, Cambridge, 2005).

[62] M. S. Hybertsen and S. G. Louie, Phys. Rev. B 34, 5390 (1986).

[63] G. Strinati, Riv. Nuovo Cimento 11, 1 (1988).

[64] M. Rohlfing and S. G. Louie, Phys. Rev. B 62, 4927 (2000).

[65] M. van Schilfgaarde, T. Kotani, and S. Faleev, Phys. Rev. Lett. 96, 226402 (2006).

[66] F. Bruneval, N. Vast, and L. Reining, Phys. Rev. B 74, 045102 (2006).

[67] T. Kotani, M. van Schilfgaarde, and S. V. Faleev, Phys. Rev. B 76, 165106 (2007).

[68] M. Shishkin and G. Kresse, Phys. Rev. B 75, 235102 (2007).

[69] R. J. Bartlett and M. Musial, Rev. Mod. Phys. 79, 291 (2007).

[70] D. Belitz and T. R. Kirkpatrick, Rev. Mod. Phys. 66, 261 (1994).

[71] C. Husemann and M. Salmhofer, Phys. Rev. B 79, 195125 (2009).

[72] M. Salmhofer and C. Honerkamp, Prog. Theor. Phys. 105, 1 (2001).

[73] T. Enss, V. Meden, S. Andergassen, X. Barnabé-Thériault, W. Metzner, and K. Schönhammer, Phys. Rev. B 71, 155401 (2005).

[74] M. Salmhofer, C. Honerkamp, W. Metzner, and O. Lauscher, Prog. Theor. Phys. 112, 943 (2004).

[75] T. Baier, E. Bick, and C. Wetterich, Phys. Rev. B 70, 125111 (2004).

[76] G. Guennebaud et al., Eigen v3, http://eigen.tuxfamily.org (2010).

[77] The HDF Group, Hierarchical Data Format, v. 5 (1997-2015), http://www.hdfgroup.org/HDF5/.

[78] OPENMP Architecture Review Board, OPENMP Application Program Interface Version 3.1, http://www.openmp.org/mpdocuments/OpenMP3.1.pdf (2011).

[79] R. Shankar, Rev. Mod. Phys. 66, 129 (1994).

[80] E. Abrahams, P. W. Anderson, D. C. Licciardello, and T. V. Ramakrishnan, Phys. Rev. Lett. 42, 673 (1979).

[81] R. Nandkishore and D. A. Huse, Annu. Rev. Condens. Matter Phys. 6, 15 (2015).

[82] N. Gemelke, Physics 9, 44 (2016).

[83] E. Cocchi, L. A. Miller, J. H. Drewes, M. Koschorreck, D. Pertot, F. Brennecke, and M. Köhl, Phys. Rev. Lett. 116, 175301 (2016).

[84] T. Vojta, F. Epperlein, and M. Schreiber, Phys. Rev. Lett. 81, 4212 (1998). 
[85] G. Benenti, X. Waintal, and J.-L. Pichard, Phys. Rev. Lett. 83, 1826 (1999).

[86] R. Berkovits, J. W. Kantelhardt, Y. Avishai, S. Havlin, and A. Bunde, Phys. Rev. B 63, 085102 (2001).

[87] R. Vlaming, G. S. Uhrig, and D. Vollhardt, J. Phys.: Condens. Matter 4, 7773 (1992).

[88] G. S. Uhrig and R. Vlaming, J. Phys.: Condens. Matter 5, 2561 (1993).

[89] M. S. Foster and A. W. W. Ludwig, Phys. Rev. B 77, 165108 (2008).

[90] W. H. Press, B. P. Flannery, S. A. Teukolsky, and W. T. Vetterling, Numerical Recipes in FORTRAN 77: The Art of
Scientific Computing (Cambridge University Press, Cambridge, 1992).

[91] M. P. Forum, MPI: A Message-Passing Interface Standard, Tech. Rep. (Knoxville, TN, USA, 1994).

[92] R. B. Lehoucq, C.-C. Yang, and D. C. Sorensen, ARPACK Users' Guide: Solution of Large-Scale Eigenvalue Problems with Implicitly Restarted Arnoldi Methods (SIAM, Philadelphia, 1998).

[93] A. Branschädel, Ph.D. dissertation, Karlsruher Institut für Technologie, 2011.

[94] A. A. Katanin, Phys. Rev. B 70, 115109 (2004).

[95] T. R. Morris, Int. J. Mod. Phys. A 09, 2411 (1994). 